\title{
Selectivity in Nickel-Catalyzed Rearrangements of Cyclopropylen-ynes
}

\author{
Gang Zuo and Janis Louie* \\ Department of Chemistry, University of Utah, 315 South 1400 East, Salt Lake City, UT 84112- \\ 0850
}

\section{SUPPORTING INFORMATION}

General information. All reactions were conducted under an atmosphere of $\mathrm{N}_{2}$ using standard Schlenk techniques or in a $\mathrm{N}_{2}$ filled glove-box unless otherwise noted. Toluene was dried over neutral alumina under $\mathrm{N}_{2}$ using a Grubbs-type solvent purification system. THF was freshly distilled from $\mathrm{Na}$ /benzophenone. $\mathrm{Ni}(\mathrm{COD})_{2}$ was purchased from Strem and used without further purification. $N$-Heterocyclic carbene ligands (e.g., ICy, ItBu, IAd, IMes, IPr) were prepared as previously reported. ${ }^{1}$ The cyclopropylen-ynes $\mathbf{1 a}, \mathbf{1 b}, \mathbf{1 e}, \mathbf{8}$ and $\mathbf{1 0}$ were synthesized using literature procedures or under slightly modified procedures. ${ }^{2}$ All other materials were purchased from commercial sources and used without further purification.

${ }^{1} \mathrm{H}$ and ${ }^{13} \mathrm{C}$ NMR spectra were recorded on a GE-300 or a GE-500 NMR and referenced to residual protiated solvent (resonances downfield to the standard are reported as positive). All ${ }^{13} \mathrm{C}$ NMR spectra were proton decoupled. Low-resolution mass spectra were obtained on a Hewlett Packard 5890 series II gas chromatograph interfaced with a Hewlett Packard 5989A mass spectrometer. IR spectra were recorded on a Bruker Tensor 27 FT-IR spectrometer. Highresolution mass spectra (HR-MS) (EI) were provided by the University of Utah Mass Spectrometry Facility.

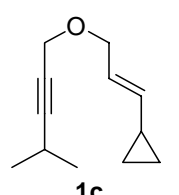

[(1E)-3-(4-methyl-2-pentynyloxy)-1-propenyl]-cyclopropane (1c).

To a stirred suspension of $\mathrm{NaH}(0.4 \mathrm{~g}, 10 \mathrm{mmol})$ and dry THF $(50 \mathrm{~mL})$ in a $100 \mathrm{~mL}$ round-bottom flask was added 3-cyclopropyl-2-propen-1-ol (0.98 g, $10 \mathrm{mmol}$ ) dropwise under $\mathrm{N}_{2}$ counter-flow. The resulting solution was stirred at room temperature for $0.5 \mathrm{~h}$ after which time 1-bromo-4-methyl-2-pentyne $(1.61 \mathrm{~g}, 10 \mathrm{~mol})$ was added. The reaction mixture was then stirred at room temperature overnight. The solution was quenched with $20 \mathrm{~mL}$ sat. $\mathrm{NH}_{4} \mathrm{Cl}$ solution. The aqueous layer was extracted $3 \times 10 \mathrm{~mL} \mathrm{Et}_{2} \mathrm{O}$. The collected organics were washed with brine, dried with anhydrous $\mathrm{MgSO}_{4}$, and concentrated in vacuo. The resulting crude yellow oil was purified by flash column chromatography (10\% $\mathrm{Et}_{2} \mathrm{O}$ /pentanes) to yield 1c (1.32 g, 74\%) as a colorless oil. ${ }^{1} \mathrm{H}$ NMR (300 $\left.\mathrm{MHz}, \mathrm{CDCl}_{3}, \mathrm{ppm}\right): \delta$ 5.62 (dt, 6.6 Hz, 15.0 Hz, 1H), 5.25 (dd, 9.0 Hz, 15.0 Hz, 1H), 4.09 (d, 2.1 Hz, 2H), 3.95 (d, 6.6 $\mathrm{Hz}, 2 \mathrm{H}), 2.58(\mathrm{~m}, 1 \mathrm{H}), 1.38(\mathrm{~m}, 1 \mathrm{H}), 1.16(\mathrm{~d}, 6.6 \mathrm{~Hz}, 6 \mathrm{H}), 0.71(\mathrm{~m}, 2 \mathrm{H}), 0.37(\mathrm{~m}, 2 \mathrm{H}) ;{ }^{13} \mathrm{C}\left\{{ }^{1} \mathrm{H}\right\}$ NMR (75 MHz, $\left.\mathrm{CDCl}_{3}, \mathrm{ppm}\right): \delta 139.2,123.1,92.1,75.1,70.0,57.3,22.9,20.5,13.4$, 6.7; IR (neat): 2872, 2935, 2851, 2249, 1668, 1461, 1357, 1320, 1184, 1080, 965, 812; HR-MS calcd. for $\mathrm{C}_{12} \mathrm{H}_{19} \mathrm{O}(\mathrm{M}+\mathrm{H}):$ 179.1436, found 179.1428 .

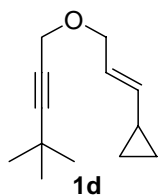

[(1E)-3-(4,4-dimethyl-2-pentynyloxy)-1-propenyl]-cyclopropane (1d).

Prepared analogously to $1 \mathrm{c}$ with $0.40 \mathrm{~g} \mathrm{NaH}$ (10 mmol), $0.98 \mathrm{~g} 3$-cyclopropyl-2propen-1-ol $(10 \mathrm{mmol})$, and $1.75 \mathrm{~g}$ 1-bromo-4,4-dimethyl-2-pentyne ${ }^{3}$ (10 mmol). Purified by column chromatography eluting with $10 \% \mathrm{Et}_{2} \mathrm{O} /$ pentanes to yield $\mathbf{1 d}$ (1.48

g, 77\%) as a colorless oil. ${ }^{1} \mathrm{H}$ NMR (300 MHz, $\left.\mathrm{CDCl}_{3}, \mathrm{ppm}\right): \delta 5.64$ (dt, $6.6 \mathrm{~Hz}, 15.3$ Hz, 1H), 5.25 (dd, 9.0 Hz, 15.3 Hz, 1H), 4.10 (s, 2H), 3.96 (d, 6.6 Hz, 2H), 1.40 (m, 1H), 1.22 (s, 9H), 0.71 (m, 2H), 0.39 (m, 2H); ${ }^{13} \mathrm{C}\left\{{ }^{1} \mathrm{H}\right\}$ NMR (75 MHz, $\left.\mathrm{CDCl}_{3}, \mathrm{ppm}\right): \delta$ 139.3, 123.2, 95.1, 74.4, 69.9, 57.3, 30.9, 27.4, 13.4, 6.7; IR (neat): 2970, 2855, 2243, 1652, 1456, 1361, 1265, 1087, 811; HR-MS calcd. for $\mathrm{C}_{13} \mathrm{H}_{21} \mathrm{O}(\mathrm{M}+\mathrm{H})$ : 193.1592, found 193.1578 . 

and dry THF $(50 \mathrm{~mL})$ in a $100 \mathrm{~mL}$ round-bottom flask was added BuLi $(4 \mathrm{~mL}, 2.5$ $\mathrm{M}$ in hexane, $10 \mathrm{mmol}$ ) dropwise at $-78^{\circ} \mathrm{C}$. The resulting solution was stirred at -78 ${ }^{\circ} \mathrm{C}$ for $0.5 \mathrm{~h}$ after which time bromomethoxy-methane $(1.37 \mathrm{~g}, 11 \mathrm{mmol})$ was added. The reaction mixture was then stirred at room temperature for $4 \mathrm{~h}$. The solution was quenched with $20 \mathrm{~mL}$ sat. $\mathrm{NH}_{4} \mathrm{Cl}$ solution. The aqueous layer was extracted $3 \times 10 \mathrm{~mL} \mathrm{Et}_{2} \mathrm{O}$. The collected organics were washed with brine, dried with anhydrous $\mathrm{MgSO}_{4}$, and concentrated in vacuo. The resulting crude yellow oil was purified by flash column chromatography (10\% $\mathrm{Et}_{2} \mathrm{O}$ /pentanes) to yield 12. (1.18 g, 66\%). ${ }^{1} \mathrm{H} \mathrm{NMR}\left(300 \mathrm{MHz}, \mathrm{CDCl}_{3}\right.$, ppm): $\delta 5.62$ (dt, $\left.6.6 \mathrm{~Hz}, 15.3 \mathrm{~Hz}, 1 \mathrm{H}\right), 5.26$ (dd, 8.7 Hz, 15.3 Hz, 1H), 4.17 (t, 1.8 Hz, 2H), 4.14 (t, 1.8 Hz, 2H), 3.98 (d, 6.6 Hz, 2H), 3.38 (s, 3H), $1.41(\mathrm{~m}, 1 \mathrm{H}), 0.72(\mathrm{~m}, 2 \mathrm{H}), 0.39(\mathrm{~m}, 2 \mathrm{H}) ;{ }^{13} \mathrm{C}\left\{{ }^{1} \mathrm{H}\right\} \mathrm{NMR}\left(75 \mathrm{MHz}, \mathrm{CDCl}_{3}, \mathrm{ppm}\right): \delta 139.9$, 123.0, 82.8, 82.1, 70.4, 60.1, 57.8, 57.1, 13.7, 7.0; IR (neat): 3003, 2934, 2849, 1666, 1449, 1351, 1188, 1098, 966, 906; HR-MS calcd. for $\mathrm{C}_{11} \mathrm{H}_{17} \mathrm{O}_{2}(\mathrm{M}+\mathrm{H})$ : 181.1229, found 181.1224.

HOY 4-cyclopropyl-3-buten-2-ol (20).

To a solution of cyclopropylacetylene (3.3 g, $50 \mathrm{mmol})$ and dry THF (100 mL) in a 250 $\mathrm{mL}$ round-bottom flask was added BuLi (20 mL, $2.5 \mathrm{M}$ in hexane, $50 \mathrm{mmol})$ dropwise at $-78{ }^{\circ} \mathrm{C}$. The resulting solution was stirred at $-78{ }^{\circ} \mathrm{C}$ for $0.5 \mathrm{~h}$ after which time acetaldehyde $(2.2 \mathrm{~g}, 50 \mathrm{~mol})$ was added. The reaction mixture was stirred at room temperature for $3 \mathrm{~h}$. The solvent was removed in vacuo and dry ether $(50 \mathrm{~mL})$ was added. A suspension of $\mathrm{LiAlH}_{4}(3.8 \mathrm{~g}, 10 \mathrm{mmol})$ in dry ether $(50 \mathrm{~mL})$ was added carefully at $0{ }^{\circ} \mathrm{C}$. The resulting solution was stirred at room temperature for $4 \mathrm{~h}$ then quenched with $100 \mathrm{~mL}$ saturated Rochelle's salt solution. The aqueous layer was extracted $3 \times 10 \mathrm{~mL} \mathrm{Et}_{2} \mathrm{O}$. The collected organics were washed with saturated Rochelle's salt solution and brine, dried with anhydrous $\mathrm{MgSO}_{4}$, and concentrated in vacuo. The resulting crude yellow oil was then distilled to yield 20 (3.5 g, 63\%). ${ }^{1} \mathrm{H}$ NMR (300 MHz, $\mathrm{CDCl}_{3}, \mathrm{ppm}$ ): $\delta 5.60$ (dd, $6.3 \mathrm{~Hz}, 15.3 \mathrm{~Hz}, 1 \mathrm{H}$ ), 5.15 (dd, 9.0 Hz, 15.3 Hz, 1H), 4.24 (m, 1H), 1.46 (br, 1H), 1.41 (m, 1H), 1.25 (d, 6.3 Hz, 3H), 0.70 (m, 2H), 0.37 (m, 2H); ${ }^{13} \mathrm{C}\left\{{ }^{1} \mathrm{H}\right\}$ NMR $\left(75 \mathrm{MHz}, \mathrm{CDCl}_{3}, \mathrm{ppm}\right): \delta 135.1,131.9,69.1,23.6,13.5$, 6.9; IR (neat): 3387, 1598, 1067; HR-MS calcd. for $\mathrm{C}_{7} \mathrm{H}_{13} \mathrm{O}(\mathrm{M}+\mathrm{H})$ : 113.0966, found 113.0959.

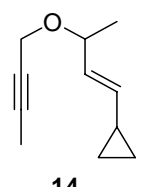

[(1E)-3-(2-butynyloxy)-1-propenyl]-cyclopropane (14).

Prepared analogously to 1c with $0.40 \mathrm{~g} \mathrm{NaH}$ (10 mmol), $1.12 \mathrm{~g} 20$ ( $10 \mathrm{mmol}$ ), and 1.33 g 1-bromo-2-butyne (10 mmol). Purified by column chromatography eluting with $10 \%$ $\mathrm{Et}_{2} \mathrm{O} /$ pentanes to yield 14 (1.47 g, 89\%). ${ }^{1} \mathrm{H}$ NMR (300 MHz, $\mathrm{CDCl}_{3}, \mathrm{ppm}$ ): $\delta 5.36$ (dd, 8.1 Hz, $15.3 \mathrm{~Hz}, 1 \mathrm{H}$ ), 5.16 (dd, $8.7 \mathrm{~Hz}, 15.3 \mathrm{~Hz}, 1 \mathrm{H}), 4.10$ (dq, $2.4 \mathrm{~Hz}, 15.0 \mathrm{~Hz}, 1 \mathrm{H}$ ), 3.93 (m, 2H), 1.84 (t, 2.4 Hz, 3H), 1.39 (m, 1H), 1.24 (d, 6.3 Hz, 3H), 0.71 (m, 2H), 0.38 (m, 2H); ${ }^{13} \mathrm{C}\left\{{ }^{1} \mathrm{H}\right\}$ NMR $\left(75 \mathrm{MHz}, \mathrm{CDCl}_{3}, \mathrm{ppm}\right): \delta 137.8,128.7,81.8,75.8,75.4,55.6,21.8,13.5,7.1,7.0$, 3.9; IR (neat): 2973, 2841, 1439, 1383, 1100, 1028, 998, 906, 858; HR-MS calcd. for $\mathrm{C}_{11} \mathrm{H}_{17} \mathrm{O}$ $(\mathrm{M}+\mathrm{H}): 165.1279$, found 165.1270 .

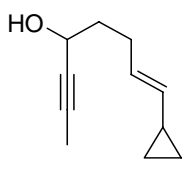

8-cyclopropyl-7-octen-2-yn-4-ol (21).

To a solution of BuLi (4 mL, $2.5 \mathrm{M}$ in hexane, $10 \mathrm{mmol})$ and dry THF (50 mL) in a $100 \mathrm{~mL}$ round-bottom flask was added propyne (approximate $0.4 \mathrm{~g}, 10 \mathrm{mmol}$ ) at $78^{\circ} \mathrm{C}$. The resulting solution was stirred at $-78^{\circ} \mathrm{C}$ for $0.5 \mathrm{~h}$ after which time 5cyclopropyl-4-pentenal (1.24 g, $10 \mathrm{~mol})$ was added. The reaction mixture was then stirred at room temperature for $3 \mathrm{~h}$. The solution was quenched with $20 \mathrm{~mL}$ sat. $\mathrm{NH}_{4} \mathrm{Cl}$ solution. The aqueous layer was extracted $3 \times 10 \mathrm{~mL} \mathrm{Et}_{2} \mathrm{O}$. The collected organics were washed with brine, dried with anhydrous $\mathrm{MgSO}_{4}$, and concentrated in vacuo. The resulting crude yellow oil was purified by flash column chromatography (20\% Et $2 \mathrm{O} /$ pentanes) to yield 21 (1.22 g, 74\%). ${ }^{1} \mathrm{H}$ NMR (300 MHz, CDCl 3 , ppm): $\delta 5.31$ (dt, $7.5 \mathrm{~Hz}, 10.5$ Hz, 1H), 4.78 (t, $9.0 \mathrm{~Hz}, 10.5$ Hz, 1H), 
4.38 (m, 1H), 2.34 (m, 2H), 1.85 (d, $2.1 \mathrm{~Hz}, 3 \mathrm{H}), 1.76$ (m, 3H), 1.60 (m, 1H), 0.74 (m, 2H), 0.33 (m, 2H); ${ }^{13} \mathrm{C}\left\{{ }^{1} \mathrm{H}\right\}$ NMR (75 MHz, $\left.\mathrm{CDCl}_{3}, \mathrm{ppm}\right): \delta 135.2,126.9,81.3,80.5,62.5,38.2,23.6,9.8$, 7.1, 3.8; IR (neat): 3357, 3081, 3005, 2922, 2859, 1650, 1423, 1153, 1064, 1019, 937, 810; HRMS calcd. for $\mathrm{C}_{11} \mathrm{H}_{17} \mathrm{O}(\mathrm{M}+\mathrm{H})$ : 165.1279 , found 165.1283 .

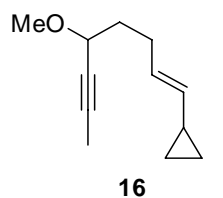

\section{1-Cyclopropyl-5-methoxy-1-octen-6-yne (16).}

To a stirred suspension of $\mathrm{NaH}(0.2 \mathrm{~g}, 5 \mathrm{mmol})$ and dry THF $(25 \mathrm{~mL})$ in a $100 \mathrm{~mL}$ round-bottom flask was added $21(0.82 \mathrm{~g}, 5 \mathrm{mmol})$ dropwise under $\mathrm{N}_{2}$ counterflow. The resulting solution was stirred at room temperature for $0.5 \mathrm{~h}$ after which time methyl iodide $(0.71 \mathrm{~g}, 5 \mathrm{~mol})$ was added. The reaction mixture was then stirred at room temperature for $3 \mathrm{~h}$. The solution was quenched with $20 \mathrm{~mL}$ sat. $\mathrm{NH}_{4} \mathrm{Cl}$ solution. The aqueous layer was extracted $3 \times 10 \mathrm{~mL} \mathrm{Et}_{2} \mathrm{O}$. The collected organics were washed with brine, dried with anhydrous $\mathrm{MgSO}_{4}$, and concentrated in vacuo. The resulting crude yellow oil was purified by flash column chromatography (10\% Et ${ }_{2} \mathrm{O} /$ pentanes) to yield $\mathbf{1 6}(0.72 \mathrm{~g}, 81 \%)$ as a colorless oil. ${ }^{1} \mathrm{H}$ NMR (300 MHz, $\mathrm{CDCl}_{3}$, ppm): $\delta 5.28$ (dt, $\left.7.5 \mathrm{~Hz}, 10.5 \mathrm{~Hz}, 1 \mathrm{H}\right), 4.76$ (t, $10.5 \mathrm{~Hz}$, 1H), 3.93 (m, 1H), 3.39 (s, 3H), 2.30 (m, 2H), 1.87 (d, $2.1 \mathrm{~Hz}, 3 \mathrm{H}), 1.75$ (m, 2H), 1.59 (m, 1H), 0.73 (m, 2H), 0.31 (m, 2H); ${ }^{13} \mathrm{C}\left\{{ }^{1} \mathrm{H}\right\}$ NMR (75 MHz, $\left.\mathrm{CDCl}_{3}, \mathrm{ppm}\right): \delta 135.0,126.9,82.0,78.2$, 71.0, 56.5, 36.1, 23.6, 9.8, 7.0, 3.8; IR (neat): 3005, 2925, 1446, 1338, 1102, 937; HR-MS calcd. for $\mathrm{C}_{12} \mathrm{H}_{19} \mathrm{O}(\mathrm{M}+\mathrm{H}):$ 179.1436, found 179.1436.

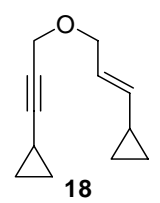

\section{[3-(3-cyclopropyl-2-pentenyloxy)-1-propynyl]-cyclopropane (18).}

Prepared analogously to 1c with $0.40 \mathrm{~g} \mathrm{NaH}$ (10 mmol), $0.98 \mathrm{~g} 3$-cyclopropyl-2propen-1-ol (10 mmol), and $1.6 \mathrm{~g}$ 3-bromo-1-propynyl-cyclopropane (10 mmol). Purified by column chromatography eluting with $10 \% \mathrm{Et}_{2} \mathrm{O} /$ pentanes to yield 1d (1.53 g, 87\%) as a colorless oil. ${ }^{1} \mathrm{H}$ NMR (300 MHz, $\left.\mathrm{CDCl}_{3}, \mathrm{ppm}\right): \delta 5.62$ (dt, $6.6 \mathrm{~Hz}, 15.3$ Hz, 1H), 5.25 (dd, 8.7 Hz, 15.3 Hz, 1H), 4.07 (d, 1.8 Hz, 2H), 3.95 (d, 6.6 Hz, 2H), 1.41 (m, 1H), $1.25(\mathrm{~m}, 1 \mathrm{H}), 0.75(\mathrm{~m}, 6 \mathrm{H}), 0.39(\mathrm{~m}, 2 \mathrm{H}) ;{ }^{13} \mathrm{C}\left\{{ }^{1} \mathrm{H}\right\} \mathrm{NMR}\left(75 \mathrm{MHz}, \mathrm{CDCl}_{3}, \mathrm{ppm}\right): \delta 139.5,123.3$, 90.1, 71.5, 70.2, 57.5, 13.7, 8.4, 7.0; IR (neat): 3085, 3008, 2850, 2247, 1357, 1074, 966, 878, 812; HR-MS calcd. for $\mathrm{C}_{12} \mathrm{H}_{17} \mathrm{O}(\mathrm{M}+\mathrm{H})$ : 177.1279, found 177.1271.

General Procedure for Ni-catalyzed Rearrangement. In a glove box, the cyclopropylen-yne was added to an oven dried scintillation vial equipped with a magnetic stir bar and dissolved in toluene. To the stirring solution, a solution of $\mathrm{Ni}(\mathrm{COD})_{2}$ and NHC ligand (previously equilibrated for at least $6 \mathrm{~h})^{4}$ was added. The reaction mixture was stirred at room temperature for $2 \mathrm{~h}$ (or until complete consumption of starting material was observed as judged by GC or TLC). The mixture was concentrated in vacuo and purified by silica gel column chromatography.

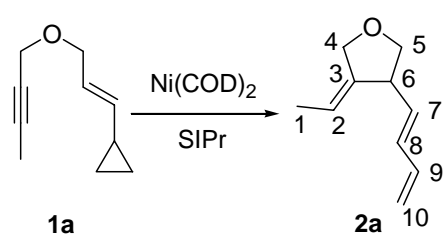

Rearrangement of [(1E)-3-(2-butynyloxy)-1-propenyl]cyclopropane (1a) catalyzed by $\mathrm{Ni}(\mathrm{COD})_{2} / \mathrm{SIPr}$. The general procedure was used with [(1E)-3-(2-butynyloxy)-1-propenyl]cyclopropane (1a, $100 \mathrm{mg}, 0.67 \mathrm{mmol}), \mathrm{Ni}(\mathrm{COD})_{2}$ (10 mg, 0.036 mmol), SIPr (14 mg, $0.036 \mathrm{mmol})$, and $0.5 \mathrm{~mL}$ of toluene. The reaction mixture was purified by column chromatography on silica gel (10\% Et $\mathrm{E}_{2} \mathrm{O}$ /pentanes) to afford 3-(1,3-butadienyl)-4-ethylidenetetrahydrofuran (2a, $57 \mathrm{mg}$, 57\%) as a colorless oil. ${ }^{1} \mathrm{H}$ NMR (300 MHz, $\left.\mathrm{CDCl}_{3}, \mathrm{ppm}\right): \delta 6.64$ (dt, $\left.10.5 \mathrm{~Hz}, 16.8 \mathrm{~Hz}, 1 \mathrm{H}\right), 6.17$ (t, 10.5 Hz, 1H), 5.21 (m, 4H), 4.37 (m, 2H), 4.10 (t, 7.8 Hz, 1H), 3.74 (m, 1H), 3.41 (t, $9.0 \mathrm{~Hz}$, $1 \mathrm{H}), 1.58(\mathrm{~m}, 3 \mathrm{H}) ;{ }^{13} \mathrm{C}\left\{{ }^{1} \mathrm{H}\right\}$ NMR $\left(75 \mathrm{MHz}, \mathrm{CDCl}_{3}, \mathrm{ppm}\right): \delta 142.1,132.2,131.5,130.4,118.7$, 115.9, 73.8, 69.6, 43.2, 14.9; IR (neat): 2851, 1668, 1363, 1070, 919; HR-MS calcd. for $\mathrm{C}_{10} \mathrm{H}_{15} \mathrm{O}$ $(\mathrm{M}+\mathrm{H}):$ 151.1123, found 172.1140 .

COSY summary; the following cross peaks were observed: $\mathrm{H}(1)$ and $\mathrm{H}(2) ; \mathrm{H}(1)$ and $\mathrm{H}(4)$; $\mathrm{H}(5)$ and $\mathrm{H}(6) ; \mathrm{H}(7)$ and $\mathrm{H}(8) ; \mathrm{H}(8)$ and $\mathrm{H}(9) ; \mathrm{H}(9)$ and $\mathrm{H}(10)$. 
HMBC summary; the following cross peaks were observed: $\mathrm{H}(1)$ and $\mathrm{C}(2), \mathrm{C}(3) ; \mathrm{H}(2)$ and $\mathrm{C}(4), \mathrm{C}(6) ; \mathrm{H}(4)$ and $\mathrm{C}(2), \mathrm{C}(3), \mathrm{C}(5), \mathrm{C}(6) ; \mathrm{H}(5)$ and $\mathrm{C}(3), \mathrm{C}(4), \mathrm{C}(6), \mathrm{C}(7) ; \mathrm{H}(6)$ and $\mathrm{C}(3) ; \mathrm{H}(7)$ and $\mathrm{C}(3), \mathrm{C}(5), \mathrm{C}(6), \mathrm{C}(8), \mathrm{C}(9) ; \mathrm{H}(8)$ and $\mathrm{C}(6), \mathrm{C}(9), \mathrm{C}(10) ; \mathrm{H}(9)$ and $\mathrm{C}(7), \mathrm{C}(8) ; \mathrm{H}(10)$ and $\mathrm{C}(8)$.

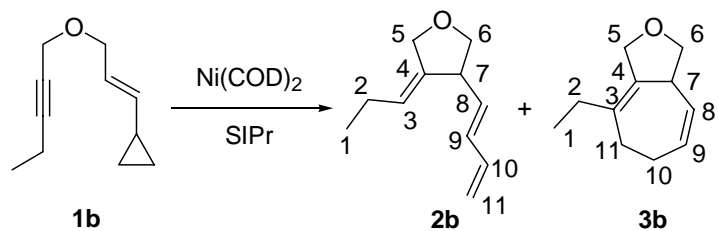

Rearrangement of [(1E)-3-(2-pentynyloxy)-1-
propenyl]-cyclopropane (1b) catalyzed by
Ni(COD) $)_{2}$ /SIPr. The general procedure was used
with [(1E)-3-(2-pentynyloxy)-1-propenyl]-
cyclopropane $^{2 \mathrm{a}}(\mathbf{1 b}, 100 \mathrm{mg}, 0.6 \mathrm{mmol}), \mathrm{Ni}(\mathrm{COD})_{2}$ ( $8 \mathrm{mg}, 0.03 \mathrm{mmol}$ ), SIPr (12 $\mathrm{mg}, 0.03 \mathrm{mmol}$ ), and $0.5 \mathrm{~mL}$ of toluene. The reaction mixture was purified by column chromatography on silica gel (10\% $\mathrm{Et}_{2} \mathrm{O}$ /pentanes) to afford 3-(1,3-butadienyl)-tetrahydro-4-propylidenefuran (2b, $34 \mathrm{mg}$, 34\%) and 8-ethyl-3,3a,6,7-tetrahydro-1H-cyclohepta[c] furan ${ }^{2 \mathrm{a}}$ (3b, $27 \mathrm{mg}, 27 \%$ ).

2b: ${ }^{1} \mathrm{H}$ NMR (300 MHz, $\left.\mathrm{CDCl}_{3}, \mathrm{ppm}\right): \delta 6.65$ (dt, $\left.10.8 \mathrm{~Hz}, 16.5 \mathrm{~Hz}, 1 \mathrm{H}\right), 6.17$ (t, $\left.10.8 \mathrm{~Hz}, 1 \mathrm{H}\right)$, 5.18 (m, 4H), 4.43 (d, 10.2 Hz, 1H), 4.32 (d, $10.2 \mathrm{~Hz}, 1 \mathrm{H}), 4.09$ (t, $8.4 \mathrm{~Hz}, 1 \mathrm{H}), 3.75$ (m, 1H), 3.40 (t, $8.4 \mathrm{~Hz}, 1 \mathrm{H}), 1.93(\mathrm{~m}, 2 \mathrm{H}), 0.96(\mathrm{t}, 7.5 \mathrm{~Hz}, 3 \mathrm{H}) ;{ }^{13} \mathrm{C}\left\{{ }^{1} \mathrm{H}\right\}$ NMR $\left(75 \mathrm{MHz}, \mathrm{CDCl}_{3}, \mathrm{ppm}\right): \delta$ 140.6, 132.2, 131.5, 130.4, 123.4, 118.7, 73.6, 69.4, 43.1, 23.1, 14.1; IR (neat): 2965, 2845, 1642, 1454, 1357, 1075, 1000, 918; HR-MS calcd. for $\mathrm{C}_{11} \mathrm{H}_{17} \mathrm{O}(\mathrm{M}+\mathrm{H})$ : 165.1279, found 165.1280 .

HMBC summary; the following cross peaks were observed: $\mathrm{H}(1)$ and $\mathrm{C}(2), \mathrm{C}(3) ; \mathrm{H}(2)$ and $\mathrm{C}(1), \mathrm{C}(3), \mathrm{C}(4) ; \mathrm{H}(3)$ and $\mathrm{C}(5), \mathrm{C}(7) ; \mathrm{H}(5)$ and $\mathrm{C}(3), \mathrm{C}(4), \mathrm{C}(6), \mathrm{C}(7) ; \mathrm{H}(6)$ and $\mathrm{C}(4), \mathrm{C}(5), \mathrm{C}(7)$, $\mathrm{C}(8)$; $\mathrm{H}(7)$ and $\mathrm{C}(4), \mathrm{C}(6), \mathrm{C}(8) ; \mathrm{H}(8)$ and $\mathrm{C}(4), \mathrm{C}(6), \mathrm{C}(7), \mathrm{C}(10) ; \mathrm{H}(9)$ and $\mathrm{C}(7), \mathrm{C}(10), \mathrm{C}(11)$; $\mathrm{H}(10)$ and $\mathrm{C}(8), \mathrm{C}(9) ; \mathrm{H}(11)$ and $\mathrm{C}(9)$.

3b ${ }^{2 \mathrm{a}}:{ }^{1} \mathrm{H}$ NMR (300 MHz, $\left.\mathrm{CDCl}_{3}, \mathrm{ppm}\right): \delta 5.70(\mathrm{~m}, 1 \mathrm{H}), 5.38(\mathrm{~m}, 1 \mathrm{H}), 4.41(\mathrm{~d}, 12.9 \mathrm{~Hz}, 1 \mathrm{H}), 4.30$ (d, $12.9 \mathrm{~Hz}, 1 \mathrm{H}), 4.21$ (t, $8.4 \mathrm{~Hz}), 3.70(\mathrm{~m}, 1 \mathrm{H}), 3.45$ (t, $8.4 \mathrm{~Hz}, 1 \mathrm{H}), 2.52(\mathrm{~m}, 1 \mathrm{H}), 2.32(\mathrm{~m}, 1 \mathrm{H})$, $2.17(\mathrm{~m}, 1 \mathrm{H}), 2.07(\mathrm{~m}, 1 \mathrm{H}), 2.01(\mathrm{q}, 7.5 \mathrm{~Hz}, 2 \mathrm{H}), 0.99(\mathrm{t}, 7.5 \mathrm{HZ}, 3 \mathrm{H}) ;{ }^{13} \mathrm{C}\left\{{ }^{1} \mathrm{H}\right\} \mathrm{NMR}(75 \mathrm{MHz}$, $\left.\mathrm{CDCl}_{3}, \mathrm{ppm}\right): \delta 135.9,132.9,131.0,128.2,75.3,70.8,41.4,29.8,28.7,27.0,12.4$; IR (neat): 2958, 2840, 1455, 1064, 938, 678; HR-MS calcd. for $\mathrm{C}_{11} \mathrm{H}_{17} \mathrm{O}(\mathrm{M}+\mathrm{H})$ : 165.1279, found 165.1285.

COSY summary; the following cross peaks were observed: $\mathrm{H}(1)$ and $\mathrm{H}(2) ; \mathrm{H}(2)$ and $\mathrm{H}(5)$; $\mathrm{H}(6)$ and $\mathrm{H}(7) ; \mathrm{H}(8)$ and $\mathrm{H}(9) ; \mathrm{H}(9)$ and $\mathrm{H}(10) ; \mathrm{H}(10)$ and $\mathrm{H}(11) ; \mathrm{H}(11)$ and $\mathrm{H}(5)$.

HMBC summary; the following cross peaks were observed: $\mathrm{H}(1)$ and $\mathrm{C}(2), \mathrm{C}(3) ; \mathrm{H}(2)$ and $\mathrm{C}(1), \mathrm{C}(2), \mathrm{C}(4), \mathrm{C}(11)$; $\mathrm{H}(5)$ and $\mathrm{C}(3), \mathrm{C}(4), \mathrm{C}(6), \mathrm{C}(7)$; H(6) and $\mathrm{C}(4), \mathrm{C}(5), \mathrm{C}(7), \mathrm{C}(8) ; \mathrm{H}(8)$ and $\mathrm{C}(4), \mathrm{C}(6), \mathrm{C}(7), \mathrm{C}(10) ; \mathrm{H}(9)$ and $\mathrm{C}(7) ; \mathrm{H}(10)$ and $\mathrm{C}(3), \mathrm{C}(8), \mathrm{C}(9), \mathrm{C}(11) ; \mathrm{H}(11)$ and $\mathrm{C}(2)$, $\mathrm{C}(3), \mathrm{C}(4), \mathrm{C}(9), \mathrm{C}(10)$.

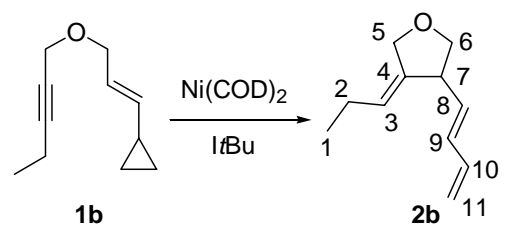

Rearrangement of [(1E)-3-(2-pentynyloxy)-1-propenyl]cyclopropane (1b) catalyzed by $\mathrm{Ni}(\mathrm{COD})_{2} / \mathrm{ItBu}$. The general procedure was used with [(1E)-3-(2-pentynyloxy)-1-propenyl]cyclopropane (1b, $100 \mathrm{mg}, 0.6 \mathrm{mmol}), \mathrm{Ni}(\mathrm{COD})_{2}(8 \mathrm{mg}, 0.03$ $\mathrm{mmol})$, ItBu (5.4 mg, $0.03 \mathrm{mmol})$, and $0.5 \mathrm{~mL}$ of toluene. The reaction mixture was purified by column chromatography on silica gel (10\% Et $2 \mathrm{O}$ /pentanes) to afford 3-(1,3-butadienyl)-tetrahydro-4-propylidenefuran (2b, 52 mg, 52\%) as a colorless oil. ${ }^{1} \mathrm{H}$ NMR (300 MHz, $\left.\mathrm{CDCl}_{3}, \mathrm{ppm}\right): \delta 6.65$ (dt, $10.8 \mathrm{~Hz}, 16.5 \mathrm{~Hz}, 1 \mathrm{H}$ ), 6.17 (t, $10.8 \mathrm{~Hz}, 1 \mathrm{H}), 5.18$ (m, 4H), 4.43 (d, $10.2 \mathrm{~Hz}, 1 \mathrm{H}), 4.32$ (d, $10.2 \mathrm{~Hz}, 1 \mathrm{H}), 4.09$ (t, $8.4 \mathrm{~Hz}$, $1 \mathrm{H}), 3.75(\mathrm{~m}, 1 \mathrm{H}), 3.40(\mathrm{t}, 8.4 \mathrm{~Hz}, 1 \mathrm{H}), 1.93(\mathrm{~m}, 2 \mathrm{H}), 0.96(\mathrm{t}, 7.5 \mathrm{~Hz}, 3 \mathrm{H}) ;{ }^{13} \mathrm{C}\left\{{ }^{1} \mathrm{H}\right\} \mathrm{NMR}(75$ $\mathrm{MHz}, \mathrm{CDCl}_{3}$, ppm): $\delta 140.6,132.2,131.5,130.4,123.4,118.7,73.6,69.4,43.1,23.1$, 14.1; IR (neat): 2965, 2845, 1642, 1454, 1357, 1075, 1000, 918; HR-MS calcd. for $\mathrm{C}_{11} \mathrm{H}_{17} \mathrm{O}(\mathrm{M}+\mathrm{H})$ : 165.1279, found 165.1280 .

HMBC summary; the following cross peaks were observed: $\mathrm{H}(1)$ and $\mathrm{C}(2), \mathrm{C}(3) ; \mathrm{H}(2)$ and $\mathrm{C}(1), \mathrm{C}(3), \mathrm{C}(4) ; \mathrm{H}(3)$ and $\mathrm{C}(5), \mathrm{C}(7) ; \mathrm{H}(5)$ and $\mathrm{C}(3), \mathrm{C}(4), \mathrm{C}(6), \mathrm{C}(7) ; \mathrm{H}(6)$ and $\mathrm{C}(4), \mathrm{C}(5), \mathrm{C}(7)$, 
$\mathrm{C}(8)$; $\mathrm{H}(7)$ and $\mathrm{C}(4), \mathrm{C}(6), \mathrm{C}(8)$; $\mathrm{H}(8)$ and $\mathrm{C}(4), \mathrm{C}(6), \mathrm{C}(7), \mathrm{C}(10)$; $\mathrm{H}(9)$ and $\mathrm{C}(7), \mathrm{C}(10), \mathrm{C}(11)$; $\mathrm{H}(10)$ and $\mathrm{C}(8), \mathrm{C}(9) ; \mathrm{H}(11)$ and $\mathrm{C}(9)$.

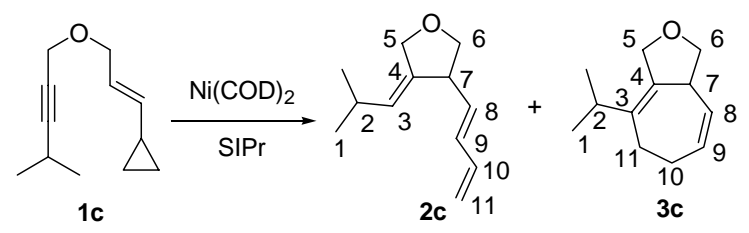

Rearrangement of [(1E)-3-(4-methyl-2-
pentynyloxy)-1-propenyl]-cyclopropane (1c)
catalyzed by Ni(COD) $/$ SIPr. The general
procedure was used with [(1E)-3-(4-methyl-2-
pentynyloxy)-1-propenyl]-cyclopropane (1c, 100
$\mathrm{mg}, 0.56 \mathrm{mmol}), \mathrm{Ni}(\mathrm{COD})_{2}(8 \mathrm{mg}, 0.03 \mathrm{mmol})$, SIPr (12 mg, $0.03 \mathrm{mmol}$ ), and $0.5 \mathrm{~mL}$ of toluene. The reaction mixture was purified by column chromatography on silica gel (10\% $\mathrm{Et}_{2} \mathrm{O}$ /pentanes) to afford 3-(1,3-butadienyl)-tetrahydro-4-(2methylpropylidene)furan (2c, $28 \mathrm{mg}, \quad 28 \%$ ) and 8-isopropyl-3,3a,6,7-tetrahydro-1Hcyclohepta[c]furan(3c, $38 \mathrm{mg}, 38 \%)$.

2c: ${ }^{1} \mathrm{H}$ NMR (300 MHz, $\left.\mathrm{CDCl}_{3}, \mathrm{ppm}\right): \delta 6.63$ (dt, $\left.10.5 \mathrm{~Hz}, 16.8 \mathrm{~Hz}, 1 \mathrm{H}\right), 6.16$ (t, $10.5 \mathrm{~Hz}, 1 \mathrm{H}$ ), $5.24(\mathrm{~m}, 3 \mathrm{H}), 4.99(\mathrm{~m}, 1 \mathrm{H}), 4.44(\mathrm{~d}, 13.2 \mathrm{~Hz}, 1 \mathrm{H}), 4.32$ (dt, $13.2 \mathrm{~Hz}, 2.1 \mathrm{~Hz}, 1 \mathrm{H}), 4.08$ (t, $8.4 \mathrm{~Hz}$, $1 \mathrm{H}), 3.74(\mathrm{~m}, 1 \mathrm{H}), 3.38(\mathrm{t}, 8.4 \mathrm{~Hz}, 1 \mathrm{H}), 2.21(\mathrm{~m}, 1 \mathrm{H}), 0.94(\mathrm{~d}, 6.6 \mathrm{~Hz}, 6 \mathrm{H}) ;{ }^{13} \mathrm{C}\left\{{ }^{1} \mathrm{H}\right\} \mathrm{NMR}(75$ $\left.\mathrm{MHz}, \mathrm{CDCl}_{3}, \mathrm{ppm}\right): \delta 139.0,132.3,131.5,130.5,129.1,118.6,73.5,69.2$, 43.0, 29.6, 23.0; IR (neat): 2958, 2849, 1463, 1359, 1067, 919; HR-MS calcd. for $\mathrm{C}_{12} \mathrm{H}_{19} \mathrm{O}(\mathrm{M}+\mathrm{H}): 179.1436$, found 179.1421.

COSY summary; the following cross peaks were observed: $\mathrm{H}(1)$ and $\mathrm{H}(2) ; \mathrm{H}(2)$ and $\mathrm{H}(3)$; $\mathrm{H}(3)$ and $\mathrm{H}(5) ; \mathrm{H}(6)$ and $\mathrm{H}(7) ; \mathrm{H}(7)$ and $\mathrm{H}(8) ; \mathrm{H}(8)$ and $\mathrm{H}(9) ; \mathrm{H}(9)$ and $\mathrm{H}(10) ; \mathrm{H}(10)$ and $\mathrm{H}(11)$.

HMBC summary; the following cross peaks were observed: $\mathrm{H}(1)$ and $\mathrm{C}(2), \mathrm{C}(3) ; \mathrm{H}(2)$ and $\mathrm{C}(1), \mathrm{C}(3), \mathrm{C}(4) ; \mathrm{H}(3)$ and $\mathrm{C}(5), \mathrm{C}(7) ; \mathrm{H}(5)$ and $\mathrm{C}(3), \mathrm{C}(4), \mathrm{C}(6), \mathrm{C}(7) ; \mathrm{H}(6)$ and $\mathrm{C}(4), \mathrm{C}(5), \mathrm{C}(7)$, $\mathrm{C}(8)$; $\mathrm{H}(7)$ and $\mathrm{C}(3), \mathrm{C}(4), \mathrm{C}(6), \mathrm{C}(8) ; \mathrm{H}(8)$ and $\mathrm{C}(4), \mathrm{C}(6), \mathrm{C}(7), \mathrm{C}(10) ; \mathrm{H}(9)$ and $\mathrm{C}(7), \mathrm{C}(10)$, $\mathrm{C}(11) ; \mathrm{H}(10)$ and $\mathrm{C}(8), \mathrm{C}(9) ; \mathrm{H}(11)$ and $\mathrm{C}(9)$.

3c: ${ }^{1} \mathrm{H}$ NMR (300 MHz, $\left.\mathrm{CDCl}_{3}, \mathrm{ppm}\right): \delta 5.64(\mathrm{~m}, 1 \mathrm{H}), 5.33(\mathrm{~m}, 1 \mathrm{H}), 4.43(\mathrm{~d}, 12.6 \mathrm{~Hz}, 1 \mathrm{H}), 4.20$ (t, $8.4 \mathrm{~Hz}$ ), 3.70 (m, 1H), 3.44 (t, $8.4 \mathrm{HZ}), 2.35$ (m, 3H), 2.10 (m, 2H), 0.99 (d, $6.6 \mathrm{~Hz}, 3 \mathrm{H}), 0.94$ (d, $6.6 \mathrm{~Hz}) ;{ }^{13} \mathrm{C}\left\{{ }^{1} \mathrm{H}\right\} \mathrm{NMR}\left(75 \mathrm{MHz}, \mathrm{CDCl}_{3}, \mathrm{ppm}\right): \delta 136.6,136.2,130.9,127.5,75.4,70.5,41.6$, 33.1, 28.0, 24.9, 20.5, 20.4; IR (neat): 2957, 2839, 1462, 1362, 1073, 937; HR-MS calcd. for $\mathrm{C}_{12} \mathrm{H}_{19} \mathrm{O}(\mathrm{M}+\mathrm{H}): 179.1436$, found 179.1407 .

COSY summary; the following cross peaks were observed: $\mathrm{H}(1)$ and $\mathrm{H}(2) ; \mathrm{H}(6)$ and $\mathrm{H}(7)$; $\mathrm{H}(8)$ and $\mathrm{H}(9) ; \mathrm{H}(9)$ and $\mathrm{H}(10) ; \mathrm{H}(10)$ and $\mathrm{H}(11) ; \mathrm{H}(11)$ and $\mathrm{H}(5)$.

HMBC summary; the following cross peaks were observed: $\mathrm{H}(1)$ and $\mathrm{C}(2), \mathrm{C}(3) ; \mathrm{H}(2)$ and $\mathrm{C}(1), \mathrm{C}(2), \mathrm{C}(4), \mathrm{C}(11) ; \mathrm{H}(5)$ and $\mathrm{C}(3), \mathrm{C}(4), \mathrm{C}(6), \mathrm{C}(7) ; \mathrm{H}(6)$ and $\mathrm{C}(4), \mathrm{C}(5), \mathrm{C}(7), \mathrm{C}(8) ; \mathrm{H}(8)$ and $\mathrm{C}(4), \mathrm{C}(6), \mathrm{C}(7), \mathrm{C}(10) ; \mathrm{H}(9)$ and $\mathrm{C}(7) ; \mathrm{H}(10)$ and $\mathrm{C}(3), \mathrm{C}(8), \mathrm{C}(9), \mathrm{C}(11) ; \mathrm{H}(11)$ and $\mathrm{C}(2)$, $\mathrm{C}(3), \mathrm{C}(4), \mathrm{C}(9), \mathrm{C}(10)$.

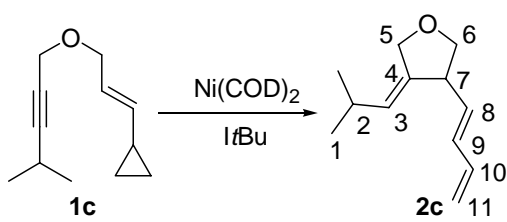

Rearrangement of [(1E)-3-(4-methyl-2-pentynyloxy)-1propenyl]-cyclopropane (1c) catalyzed by $\mathrm{Ni}(\mathrm{COD})_{2} / \mathrm{ItBu}$. The general procedure was used with [(1E)-3-(4-methyl-2pentynyloxy)-1-propenyl]-cyclopropane (1c, $100 \mathrm{mg}, \quad 0.56$ $\mathrm{mmol})$, $\mathrm{Ni}(\mathrm{COD})_{2}$ (8 mg, $\left.0.03 \mathrm{mmol}\right), \mathrm{ItBu}(5.4 \mathrm{mg}, 0.03 \mathrm{mmol})$, and $0.5 \mathrm{~mL}$ of toluene. The reaction mixture was purified by column chromatography on silica gel (10\% $\mathrm{Et}_{2} \mathrm{O} /$ pentanes) to afford 3-(1,3-butadienyl)tetrahydro-4-(2-methylpropylidene)furan (2c, $65 \mathrm{mg}, 65 \%$ ) as a colorless oil. ${ }^{1} \mathrm{H}$ NMR (300 MHz, $\left.\mathrm{CDCl}_{3}, \mathrm{ppm}\right): \delta 6.63(\mathrm{dt}, 10.5 \mathrm{~Hz}, 16.8 \mathrm{~Hz}, 1 \mathrm{H}), 6.16(\mathrm{t}, 10.5 \mathrm{~Hz}, 1 \mathrm{H}), 5.24(\mathrm{~m}, 3 \mathrm{H}), 4.99$ (m, 1H), 4.44 (d, $13.2 \mathrm{~Hz}, 1 \mathrm{H}), 4.32$ (dt, $13.2 \mathrm{~Hz}, 2.1 \mathrm{~Hz}, 1 \mathrm{H}), 4.08$ (t, $8.4 \mathrm{~Hz}, 1 \mathrm{H}), 3.74$ (m, 1H), 3.38 (t, $8.4 \mathrm{~Hz}, 1 \mathrm{H}), 2.21(\mathrm{~m}, 1 \mathrm{H}), 0.94(\mathrm{~d}, 6.6 \mathrm{~Hz}, 6 \mathrm{H}) ;{ }^{13} \mathrm{C}\left\{{ }^{1} \mathrm{H}\right\} \mathrm{NMR}\left(75 \mathrm{MHz}, \mathrm{CDCl}_{3}, \mathrm{ppm}\right): \delta 139.0$, 132.3, 131.5, 130.5, 129.1, 118.6, 73.5, 69.2, 43.0, 29.6, 23.0; IR (neat): 2958, 2849, 1463, 1359, 1067, 919; HR-MS calcd. for $\mathrm{C}_{12} \mathrm{H}_{19} \mathrm{O}(\mathrm{M}+\mathrm{H})$ : 179.1436, found 179.1421. 
COSY summary; the following cross peaks were observed: $H(1)$ and $H(2) ; H(2)$ and $H(3)$; $\mathrm{H}(3)$ and $\mathrm{H}(5) ; \mathrm{H}(6)$ and $\mathrm{H}(7) ; \mathrm{H}(7)$ and $\mathrm{H}(8) ; \mathrm{H}(8)$ and $\mathrm{H}(9) ; \mathrm{H}(9)$ and $\mathrm{H}(10) ; \mathrm{H}(10)$ and $\mathrm{H}(11)$.

HMBC summary; the following cross peaks were observed: $\mathrm{H}(1)$ and $\mathrm{C}(2), \mathrm{C}(3) ; \mathrm{H}(2)$ and $\mathrm{C}(1), \mathrm{C}(3), \mathrm{C}(4) ; \mathrm{H}(3)$ and $\mathrm{C}(5), \mathrm{C}(7) ; \mathrm{H}(5)$ and $\mathrm{C}(3), \mathrm{C}(4), \mathrm{C}(6), \mathrm{C}(7) ; \mathrm{H}(6)$ and $\mathrm{C}(4), \mathrm{C}(5), \mathrm{C}(7)$, $\mathrm{C}(8)$; $\mathrm{H}(7)$ and $\mathrm{C}(3), \mathrm{C}(4), \mathrm{C}(6), \mathrm{C}(8)$; $\mathrm{H}(8)$ and $\mathrm{C}(4), \mathrm{C}(6), \mathrm{C}(7), \mathrm{C}(10) ; \mathrm{H}(9)$ and $\mathrm{C}(7), \mathrm{C}(10)$, $\mathrm{C}(11) ; \mathrm{H}(10)$ and $\mathrm{C}(8), \mathrm{C}(9) ; \mathrm{H}(11)$ and $\mathrm{C}(9)$.

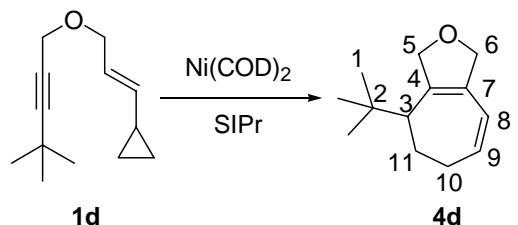

1d

Rearrangement of [(1E)-3-(4,4-dimethyl-2-pentynyloxy)-1propenyl]-cyclopropane (1d) catalyzed by $\mathrm{Ni}(\mathrm{COD})_{2} / \mathrm{SIPr}$. The general procedure was used with [(1E)-3-(4,4-dimethyl-2pentynyloxy)-1-propenyl]-cyclopropane (1d, $100 \mathrm{mg}, 0.5$ mmol), $\mathrm{Ni}(\mathrm{COD})_{2}$ (8 mg, $\left.0.03 \mathrm{mmol}\right)$, SIPr (11 mg, $0.03 \mathrm{mmol}$ ), and $0.5 \mathrm{~mL}$ of toluene. The reaction mixture was purified by column chromatography on silica gel (10\% $\mathrm{Et}_{2} \mathrm{O} /$ pentanes) to afford 8-(1',1'-dimethyl-ethyl)1,3,6,7-tetrahydro-2H-cyclohepta[c]furan (4d, $82 \mathrm{mg}, 82 \%)$ as a colorless oil. ${ }^{1} \mathrm{H}$ NMR (300 MHz, $\left.\mathrm{CDCl}_{3}, \mathrm{ppm}\right): \delta 5.82(\mathrm{~m}, 1 \mathrm{H}), 5,69(\mathrm{~d}, 11.4 \mathrm{~Hz}, 1 \mathrm{H}), 4.96(\mathrm{~m}, 1 \mathrm{H}), 4.80(\mathrm{~m}, 2 \mathrm{H}), 4.60(\mathrm{~m}$, 1H), $2.51(\mathrm{~m}, 2 \mathrm{H}), 2.19(\mathrm{~m}, 1 \mathrm{H}), 1.62(\mathrm{~m}, 1 \mathrm{H}), 0.95(\mathrm{~s}, 9 \mathrm{H}) ;{ }^{13} \mathrm{C}\left\{{ }^{1} \mathrm{H}\right\}$ NMR $\left(75 \mathrm{MHz}, \mathrm{CDCl}_{3}\right.$, ppm): $\delta$ 138.6, 134.5, 130.2, 119.6, 81.9, 79.1, 47.4, 35.7, 30.0, 29.4, 27.6; IR (neat): 2964, 2852, 1645, 1432, 1048, 922; HR-MS calcd. for $\mathrm{C}_{13} \mathrm{H}_{21} \mathrm{O}(\mathrm{M}+\mathrm{H})$ : 193.1592, found 193.1596.

COSY summary; the following cross peaks were observed: $\mathrm{H}(3)$ and $\mathrm{H}(11) ; \mathrm{H}(8)$ and $\mathrm{H}(9) ; \mathrm{H}(9)$ and $\mathrm{H}(10) ; \mathrm{H}(10)$ and $\mathrm{H}(11)$.

HMBC summary; the following cross peaks were observed: $\mathrm{H}(1)$ and $\mathrm{C}(2), \mathrm{C}(3) ; \mathrm{H}(3)$ and $\mathrm{C}(1), \mathrm{C}(2), \mathrm{C}(4), \mathrm{C}(5), \mathrm{C}(7), \mathrm{C}(10)$; $\mathrm{H}(5)$ and $\mathrm{C}(4), \mathrm{C}(6), \mathrm{C}(7) ; \mathrm{H}(6)$ and $\mathrm{C}(4), \mathrm{C}(5), \mathrm{C}(7) ; \mathrm{H}(8)$ and $\mathrm{C}(4), \mathrm{C}(6), \mathrm{C}(10) ; \mathrm{H}(9)$ and $\mathrm{C}(7), \mathrm{C}(10) ; \mathrm{H}(10)$ and $\mathrm{C}(3), \mathrm{C}(8), \mathrm{C}(9) ; \mathrm{H}(11)$ and $\mathrm{C}(2), \mathrm{C}(3)$, $\mathrm{C}(4), \mathrm{C}(9), \mathrm{C}(10)$.

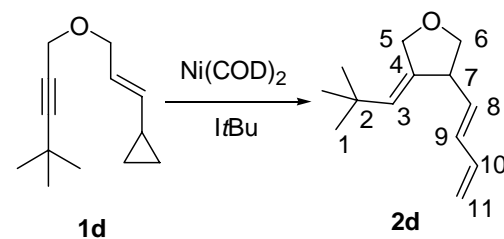

Rearrangement of [(1E)-3-(4,4-dimethyl-2-pentynyloxy)-1propenyl]-cyclopropane (1d) catalyzed by $\mathrm{Ni}(\mathrm{COD})_{2} / \mathrm{ItBu}$. The general procedure was used with [(1E)-3-(4,4-dimethyl-2pentynyloxy)-1-propenyl]-cyclopropane $\quad(\mathbf{1 d}, 100 \mathrm{mg}, 0.5$ mmol), $\mathrm{Ni}(\mathrm{COD})_{2}(8 \mathrm{mg}, 0.03 \mathrm{mmol}), \mathrm{ItBu}(5.4 \mathrm{mg}, 0.03$ $\mathrm{mmol})$, and $0.5 \mathrm{~mL}$ of toluene. The reaction mixture was purified by column chromatography on silica gel $\left(10 \% \mathrm{Et}_{2} \mathrm{O} /\right.$ pentanes) to afford 3-(1,3butadienyl)-tetrahydro-4-(2,2-dimethylpropylidene)furan (2d, $79 \mathrm{mg}, 79 \%)$ as a colorless oil. ${ }^{1} \mathrm{H}$ NMR (300 MHz, $\mathrm{CDCl}_{3}$, ppm): $\delta 6.63$ (dt, $\left.10.5 \mathrm{~Hz}, 16.8 \mathrm{~Hz}, 1 \mathrm{H}\right), 6.17$ (t, $\left.16.8 \mathrm{~Hz}, 1 \mathrm{H}\right), 5.18$ (m, 4H), 4.57 (m, 1H), 4.42 (dt, 2.4 Hz, $13.2 \mathrm{~Hz}, 1 \mathrm{H}), 4.02$ (t, $8.4 \mathrm{~Hz}, 1 \mathrm{H}), 3.73$ (m, 1H), 3.30 (t, 8.4 $\mathrm{Hz}, 1 \mathrm{H}), 1.03$ (s, 9H); ${ }^{13} \mathrm{C}\left\{{ }^{1} \mathrm{H}\right\} \mathrm{NMR}\left(75 \mathrm{MHz}, \mathrm{CDCl}_{3}\right.$, ppm): $\delta 138.0,132.7,132.2,131.6,130.9$, 118.7, 72.4, 68.9, 44.6, 33.5, 30.4; IR (neat): 2960, 2858, 1596, 1360, 1078, 935; HR-MS calcd. for $\mathrm{C}_{13} \mathrm{H}_{21} \mathrm{O}(\mathrm{M}+\mathrm{H}):$ 193.1592, found 193.1580.

COSY summary; the following cross peaks were observed: $\mathrm{H}(6)$ and $\mathrm{H}(7) ; \mathrm{H}(7)$ and $\mathrm{H}(8)$; $\mathrm{H}(8)$ and $\mathrm{H}(9) ; \mathrm{H}(9)$ and $\mathrm{H}(10) ; \mathrm{H}(10)$ and $\mathrm{H}(11)$.

HMBC summary; the following cross peaks were observed: $\mathrm{H}(1)$ and $\mathrm{C}(2), \mathrm{C}(3) ; \mathrm{H}(3)$ and C(5), C(7); H(5) and C(3), C(4), C(6); H(6) and C(4), C(5), C(7), C(8); H(7) and C(4), C(6), C(8); $\mathrm{H}(8)$ and $\mathrm{C}(4), \mathrm{C}(6), \mathrm{C}(10) ; \mathrm{H}(9)$ and $\mathrm{C}(10), \mathrm{C}(11) ; \mathrm{H}(10)$ and $\mathrm{C}(8), \mathrm{C}(9) ; \mathrm{H}(11)$ and $\mathrm{C}(9)$.

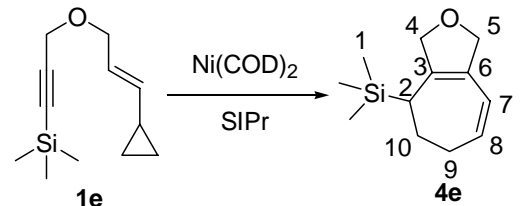

Rearrangement of [3-[[(2E)-3-cyclopropyl-2-propenyl]oxy]1-propynyl]trimethylsilane (1e) catalyzed by $\mathrm{Ni}(\mathrm{COD})_{2} / \mathrm{SIPr}$. The general procedure was used with [3-[[(2E)-3-cyclopropyl-2propenyl]oxy]-1-propynyl]trimethylsilane (1e, $100 \mathrm{mg}, 0.5$ mmol), Ni(COD) 2 (8 mg, $0.03 \mathrm{mmol}), \operatorname{SIPr}(11 \mathrm{mg}, 0.03 \mathrm{mmol})$, and $0.5 \mathrm{~mL}$ of toluene. The reaction mixture was purified by column chromatography on silica gel (10\% Et $2 \mathrm{O} /$ pentanes) to afford $\mathbf{4 e}(88 \mathrm{mg}, 88 \%)$ as a colorless oil. ${ }^{1} \mathrm{H} \mathrm{NMR}\left(300 \mathrm{MHz}, \mathrm{CDCl}_{3}\right.$, 
ppm): $\delta 5.74$ (m, 1H), 5.68 (m, 1H), $4.70(\mathrm{~m}, 3 \mathrm{H}), 4.55$ (m, 1H), 2.47 (m, 2H), $2.04(\mathrm{~m}, 1 \mathrm{H}), 1.89$ (m, 2H), 0.04 (s, 9H); ${ }^{13} \mathrm{C}\left\{{ }^{1} \mathrm{H}\right\}$ NMR $\left(75 \mathrm{MHz}, \mathrm{CDCl}_{3}, \mathrm{ppm}\right): \delta$ 138.3, 132.8, 126.0, 119.9, 80.5, 79.3, 31.4, 30.2, 26.1, -1.4; IR (neat): 3014, 2840, 1756, 1655, 1427, 1357, 1252, 1062, 930, 844; HR-MS calcd. for $\mathrm{C}_{12} \mathrm{H}_{21} \mathrm{OSi}(\mathrm{M}+\mathrm{H})$ : 209.1362, found 209.1340 .

COSY summary; the following cross peaks were observed: $\mathrm{H}(2)$ and $\mathrm{H}(10) ; \mathrm{H}(7)$ and $\mathrm{H}(8) ; \mathrm{H}(8)$ and $\mathrm{H}(9) ; \mathrm{H}(9)$ and $\mathrm{H}(10)$.

HMBC summary; the following cross peaks were observed: $\mathrm{H}(2)$ and $\mathrm{C}(3), \mathrm{C}(6), \mathrm{C}(9)$, $\mathrm{C}(10) ; \mathrm{H}(4)$ and $\mathrm{C}(3), \mathrm{C}(5), \mathrm{C}(6) ; \mathrm{H}(5)$ and $\mathrm{C}(3), \mathrm{C}(4), \mathrm{C}(6), \mathrm{C}(7) ; \mathrm{H}(7)$ and $\mathrm{C}(3), \mathrm{C}(5), \mathrm{C}(9)$; $\mathrm{H}(8)$ and $\mathrm{C}(6), \mathrm{C}(9), \mathrm{C}(10) ; \mathrm{H}(9)$ and $\mathrm{C}(7), \mathrm{C}(8), \mathrm{C}(10) ; \mathrm{H}(10)$ and $\mathrm{C}(2), \mathrm{C}(3), \mathrm{C}(8), \mathrm{C}(9)$.

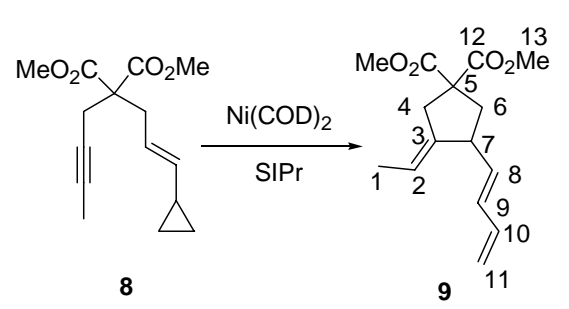

Rearrangement of [(2E)-3-cyclopropyl-2-propenyl](2butynyl)-malonic acid dimethyl ester (8) catalyzed by $\mathbf{N i ( C O D )})_{2} / \mathbf{S I P r}$. The general procedure was used with [(2E)3-cyclopropyl-2-propenyl](2-butynyl)-malonic acid dimethyl ester (8, $100 \mathrm{mg}, 0.38 \mathrm{mmol}), \mathrm{Ni}(\mathrm{COD})_{2}$ (5 mg, $\left.0.018 \mathrm{mmol}\right)$, SIPr (7 mg, $0.018 \mathrm{mmol})$, and $0.5 \mathrm{~mL}$ of toluene. The reaction mixture was purified by column chromatography on silica gel (10\% $\mathrm{Et}_{2} \mathrm{O} /$ pentanes) to afford 3-(1,3-butadienyl)-4-ethylidene-1,1-cyclopentanedicarboxylic acid dimethyl ester (9, $82 \mathrm{mg}, 82 \%$ ) as a colorless oil. ${ }^{1} \mathrm{H} \mathrm{NMR}$ (300 $\left.\mathrm{MHz}, \mathrm{CDCl}_{3}, \mathrm{ppm}\right): \delta 6.59$ (dt, $10.8 \mathrm{~Hz}, 16.8 \mathrm{~Hz}, 1 \mathrm{H}), 6.11$ (t, $10.8 \mathrm{~Hz}, 1 \mathrm{H}), 5.18$ (m, 4H), 3.76 (s, 3H), 3.74 (s, 3H), 3.59 (m, 1H), 3.04 (m, 1H), 2.86 (m, 1H), 2.56 (ddd, $1.2 \mathrm{~Hz}, 7.2 \mathrm{~Hz}, 12.3 \mathrm{~Hz}, 1 \mathrm{H}), 1.88$ (t, $12.3 \mathrm{~Hz}, 1 \mathrm{H}$ ), 1.59 (dd, $1.8 \mathrm{~Hz}, 6.6 \mathrm{~Hz}, 3 \mathrm{H}) ;{ }^{13} \mathrm{C}\left\{{ }^{1} \mathrm{H}\right\} \mathrm{NMR}\left(75 \mathrm{MHz}, \mathrm{CDCl}_{3}, \mathrm{ppm}\right): \delta 172.3,172.2,141.1$, 133.3, 132.3, 130.5, 118.0, 58.6, 52.9, 52.8, 41.7, 40.9, 36.7, 14.6; IR (neat): 2955, 1738, 1438, 1264, 1170, 912; HR-MS calcd. for $\mathrm{C}_{15} \mathrm{H}_{21} \mathrm{O}_{4}(\mathrm{M}+\mathrm{H})$ : 265.1440, found 265.1429.

COSY summary; the following cross peaks were observed: $\mathrm{H}(1)$ and $\mathrm{H}(2) ; \mathrm{H}(1)$ and $\mathrm{H}(4)$; $\mathrm{H}(6)$ and $\mathrm{H}(7) ; \mathrm{H}(7)$ and $\mathrm{H}(8) ; \mathrm{H}(8)$ and $\mathrm{H}(9) ; \mathrm{H}(9)$ and $\mathrm{H}(10) ; \mathrm{H}(10)$ and $\mathrm{H}(11)$.

HMBC summary; the following cross peaks were observed: $\mathrm{H}(1)$ and $\mathrm{C}(2), \mathrm{C}(3) ; \mathrm{H}(2)$ and $\mathrm{C}(4)$; $\mathrm{H}(4)$ and $\mathrm{C}(2), \mathrm{C}(3), \mathrm{C}(5), \mathrm{C}(6), \mathrm{C}(12) ; \mathrm{H}(6)$ and $\mathrm{C}(3), \mathrm{C}(4), \mathrm{C}(5), \mathrm{C}(7), \mathrm{C}(12) ; \mathrm{H}(8)$ and C(3), C(6), C(10); H(9) and C(7), C(10), C(11); H(10) and C(8), C(9); H(11) and C(9); H(13) and $\mathrm{C}(5), \mathrm{C}(12)$.

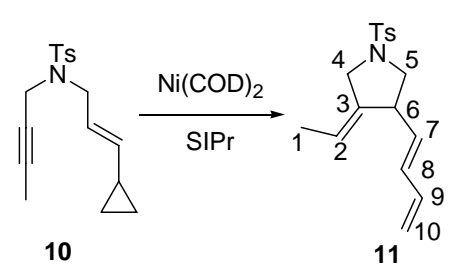

Rearrangement of N-2-butynyl-N-[(2E)-3-cyclopropyl-2propenyl]-4-methyl-benzenesulfonamide (10) catalyzed by Ni(COD) 2 /SIPr. The general procedure was used with N-2-butynyl$\mathrm{N}$-[(2E)-3-cyclopropyl-2-propenyl]-4-methyl-benzenesulfonamide (10, $100 \mathrm{mg}, 0.3 \mathrm{mmol}$ ), Ni(COD) 2 (5 mg, $0.018 \mathrm{mmol})$, SIPr (7 mg, $0.018 \mathrm{mmol})$, and $0.5 \mathrm{~mL}$ of toluene. The reaction mixture was purified by column chromatography on silica gel (25\% $\mathrm{Et}_{2} \mathrm{O} /$ pentanes) to afford 11 (88 mg, 88\%) as a white crystalline solid. ${ }^{1} \mathrm{H}$ NMR $\left(300 \mathrm{MHz} \mathrm{CDCl}_{3}, \mathrm{ppm}\right): \delta 7.72$ (d, $\left.8.1 \mathrm{~Hz}, 2 \mathrm{H}\right), 7.35$ (d, 8.1 $\mathrm{Hz}, 2 \mathrm{H}), 6.50$ (dt, $10.5 \mathrm{~Hz}, 16.5 \mathrm{~Hz}, 1 \mathrm{H}), 6.12$ (t, $10.5 \mathrm{~Hz}, 1 \mathrm{H}), 5.26$ (d, $16.5 \mathrm{~Hz}, 1 \mathrm{H}), 5.15$ (m, $3 \mathrm{H}), 3.98(\mathrm{~m}, 1 \mathrm{H}), 3.66(\mathrm{~m}, 3 \mathrm{H}), 2.66(\mathrm{~m}, 1 \mathrm{H}), 2.45(\mathrm{~s}, 3 \mathrm{H}), 1.53(\mathrm{~m}, 3 \mathrm{H}) ;{ }^{13} \mathrm{C}\{1 \mathrm{H}\} \mathrm{NMR}(75$ $\mathrm{MHz}, \mathrm{CDCl}_{3}$, ppm): $\delta 143.9,138.2,133.0,132.2,131.8,129.9,129.4,128.1,119.4,118.6,53.6$, 49.7, 41.8, 21.8, 14.7; IR (neat): 2925, 2856, 1706, 1597, 1447, 1346, 1162, 1099, 1025, 911, 818, 623, 588, 548; HR-MS calcd. For $\mathrm{C}_{17} \mathrm{H}_{22} \mathrm{NO}_{2} \mathrm{~S}(\mathrm{M}+\mathrm{H})$ : 304.1371, found 304.1367.

COSY summary; the following cross peaks were observed: $\mathrm{H}(1)$ and $\mathrm{H}(2) ; \mathrm{H}(1)$ and $\mathrm{H}(4)$; $\mathrm{H}(2)$ and $\mathrm{H}(4) ; \mathrm{H}(5)$ and $\mathrm{H}(6) ; \mathrm{H}(6)$ and $\mathrm{H}(7) ; \mathrm{H}(7)$ and $\mathrm{H}(8) ; \mathrm{H}(8)$ and $\mathrm{H}(9) ; \mathrm{H}(9)$ and $\mathrm{H}(10)$.

HMBC summary; the following cross peaks were observed: $\mathrm{H}(1)$ and $\mathrm{C}(2), \mathrm{C}(3) ; \mathrm{H}(4)$ and $\mathrm{C}(2), \mathrm{C}(3) ; \mathrm{H}(5)$ and $\mathrm{C}(3), \mathrm{C}(6), \mathrm{C}(7)$; $\mathrm{H}(7)$ and $\mathrm{C}(5), \mathrm{C}(6), \mathrm{C}(9) ; \mathrm{H}(8)$ and $\mathrm{C}(6), \mathrm{C}(9), \mathrm{C}(10)$; $\mathrm{H}(9)$ and $\mathrm{C}(7), \mathrm{C}(8) ; \mathrm{H}(10)$ and $\mathrm{C}(8)$.

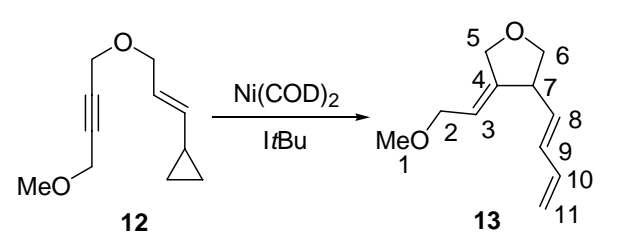

Rearrangement of [(1E)-3-(4-methoxy-2-pentynyloxy)1-propenyl]-cyclopropane

(12) catalyzed by 
$\mathbf{N i}(\mathbf{C O D})_{2} / \mathbf{I t B u}$. The general procedure was used with [(1E)-3-(4-methoxy-2-pentynyloxy)-1propenyl]-cyclopropane (12, $100 \mathrm{mg}, 0.56 \mathrm{mmol}), \mathrm{Ni}(\mathrm{COD})_{2}$ (8 mg, $\left.0.03 \mathrm{mmol}\right)$, ItBu (5.4 mg, $0.03 \mathrm{mmol}$ ), and $0.5 \mathrm{~mL}$ of toluene. The reaction mixture was purified by column chromatography on silica gel (10\% $\mathrm{Et}_{2} \mathrm{O} /$ pentanes) to afford 3-(1,3-butadienyl)-tetrahydro-4-(2methoxypropylidene)furan (13, $66 \mathrm{mg}, 66 \%$ ) as a colorless oil. ${ }^{1} \mathrm{H}$ NMR (300 $\mathrm{MHz}, \mathrm{CDCl}_{3}$, ppm): $\delta 6.63$ (dt, $10.8 \mathrm{~Hz}, 16.8 \mathrm{~Hz}, 1 \mathrm{H}), 6.20$ (t, $10.8 \mathrm{~Hz}, 1 \mathrm{H}), 5.34$ (m, 4H), 4.49 (d, $13.8 \mathrm{~Hz}, 1 \mathrm{H}), 4.37$ (d, $13.2 \mathrm{~Hz}, 1 \mathrm{H}), 4.12$ (t, $8.4 \mathrm{~Hz}, 1 \mathrm{H}), 3.85(\mathrm{~m}, 3 \mathrm{H}), 3.41(\mathrm{t}, 8.4 \mathrm{~Hz}, 1 \mathrm{H}), 3.32(\mathrm{~s}, 3 \mathrm{H}) ;{ }^{13} \mathrm{C}\left\{{ }^{1} \mathrm{H}\right\}$ NMR (75 MHz, $\mathrm{CDCl}_{3}$, ppm): $\delta$ 145.7, 132.15, 132.06, 119.1, 117.9, 73.3, 70.2, 69.4, 58.4, 43.6; IR (neat): 2978, 2844, 1596, 1440, 1381, 1195, 1115, 1073, 999, 912; HR-MS calcd. for $\mathrm{C}_{11} \mathrm{H}_{17} \mathrm{O}_{2}$ $(\mathrm{M}+\mathrm{H})$ : 181.1229, found 181.1224.

COSY summary; the following cross peaks were observed: $H(2)$ and $H(3) ; H(6)$ and $H(7)$; $\mathrm{H}(7)$ and $\mathrm{H}(8) ; \mathrm{H}(8)$ and $\mathrm{H}(9) ; \mathrm{H}(9)$ and $\mathrm{H}(10) ; \mathrm{H}(10)$ and $\mathrm{H}(11)$.

HMBC summary; the following cross peaks were observed: $\mathrm{H}(1)$ and $\mathrm{C}(2) ; \mathrm{H}(2)$ and $\mathrm{C}(1)$, $\mathrm{C}(3), \mathrm{C}(4) ; \mathrm{H}(5)$ and $\mathrm{C}(3), \mathrm{C}(4), \mathrm{C}(6) ; \mathrm{H}(6)$ and $\mathrm{C}(4), \mathrm{C}(5), \mathrm{C}(8) ; \mathrm{H}(8)$ and $\mathrm{C}(6) ; \mathrm{H}(9)$ and $\mathrm{C}(7)$, $\mathrm{C}(10), \mathrm{C}(11) ; \mathrm{H}(10)$ and $\mathrm{C}(8), \mathrm{C}(9)$.

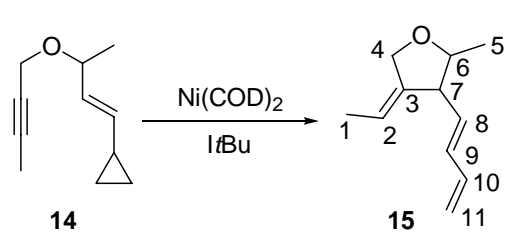

Rearrangement of [(1E)-3-(2-butynyloxy)-1-propenyl]cyclopropane (14) catalyzed by $\mathrm{Ni}(\mathrm{COD})_{2} / \mathrm{ItBu}$. The general procedure was used with [(1E)-3-(2-butynyloxy)-1-propenyl]cyclopropane (14, $100 \mathrm{mg}, 0.61 \mathrm{mmol}), \mathrm{Ni}(\mathrm{COD})_{2}(8 \mathrm{mg}, 0.03$ $\mathrm{mmol}$ ), ItBu (5.4 mg, $0.03 \mathrm{mmol}$ ), and $0.5 \mathrm{~mL}$ of toluene. The reaction mixture was purified by column chromatography on silica gel (10\% Et $2 \mathrm{O}$ /pentanes) to afford 3-(1,3-butadienyl)-tetrahydro-2-methyl-4-propylidenefuran (15, $64 \mathrm{mg}, 64 \%)$ as a colorless oil. ${ }^{1} \mathrm{H}$ NMR (300 MHz, $\left.\mathrm{CDCl}_{3}, \mathrm{ppm}\right): \delta 6.60$ (dt, $10.5 \mathrm{~Hz}$, $17.1 \mathrm{~Hz}, 1 \mathrm{H}), 6.23$ (t, $10.5 \mathrm{~Hz}, 1 \mathrm{H}), 5.27$ (d, $17.1 \mathrm{~Hz}, 1 \mathrm{H}), 5.15$ (m, 3H), 4.52 (d, $13.2 \mathrm{~Hz}, 1 \mathrm{H})$, 4.34 (dt, $13.2 \mathrm{~Hz}, 1.5 \mathrm{~Hz}, 1 \mathrm{H}), 3.56$ (m, 1H), 3.22 (m, 1H), 1.56 (m, 3H), 1.27 (d, $6.0 \mathrm{~Hz}, 3 \mathrm{H}) ;{ }^{13} \mathrm{C}$ $\left\{{ }^{1} \mathrm{H}\right\}$ NMR (75 MHz, $\left.\mathrm{CDCl}_{3}, \mathrm{ppm}\right): \delta$ 143.0, 132.5, 132.4, 129.9, 118.7, 115.7, 81.0, 69.5, 50.7, 18.8, 14.5; IR (neat): 3082, 2977, 2925, 2857, 1664, 1443, 1369, 1136, 1070, 962, 861; HR-MS calcd. for $\mathrm{C}_{11} \mathrm{H}_{17} \mathrm{O}(\mathrm{M}+\mathrm{H})$ : 165.1279 , found 165.1277 .

COSY summary; the following cross peaks were observed: $H(1)$ and $H(2) ; H(5)$ and $H(6)$; $\mathrm{H}(6)$ and $\mathrm{H}(7) ; \mathrm{H}(7)$ and $\mathrm{H}(8) ; \mathrm{H}(8)$ and $\mathrm{H}(9) ; \mathrm{H}(9)$ and $\mathrm{H}(10) ; \mathrm{H}(10)$ and $\mathrm{H}(11)$.

HMBC summary; the following cross peaks were observed: $\mathrm{H}(1)$ and $\mathrm{C}(2), \mathrm{C}(3) ; \mathrm{H}(4)$ and $\mathrm{C}(2), \mathrm{C}(3), \mathrm{C}(6) ; \mathrm{H}(5)$ and $\mathrm{C}(6), \mathrm{C}(7) ; \mathrm{H}(6)$ and $\mathrm{C}(8) ; \mathrm{H}(8)$ and $\mathrm{C}(6), \mathrm{C}(7), \mathrm{C}(10) ; \mathrm{H}(9)$ and $\mathrm{C}(7)$, $\mathrm{C}(10), \mathrm{C}(11) ; \mathrm{H}(10)$ and $\mathrm{C}(8), \mathrm{C}(9)$.

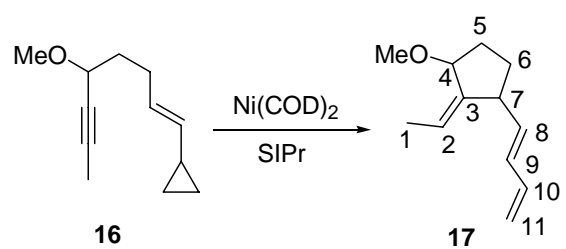

Rearrangement of 1-Cyclopropyl-5-methoxy-1-octen-6yne (16) catalyzed by $\mathrm{Ni}(\mathrm{COD})_{2} / \mathbf{I t B u}$. The general procedure was used with 1-Cyclopropyl-5-methoxy-1-octen6-yne (16, $100 \mathrm{mg}, 0.56 \mathrm{mmol}), \mathrm{Ni}(\mathrm{COD})_{2}$ (8 mg, 0.03 $\mathrm{mmol}$ ), SIPr (11 mg, $0.03 \mathrm{mmol}$ ), and $0.5 \mathrm{~mL}$ of toluene. The reaction mixture was purified by column chromatography on

silica gel (10\% $\mathrm{Et}_{2} \mathrm{O}$ /pentanes) to afford 1-(1,3-butadienyl)-3-methoxy-2-propylidenecyclopentane (17, $58 \mathrm{mg}, 58 \%$ ) as a colorless oil. ${ }^{1} \mathrm{H}$ NMR (300 $\left.\mathrm{MHz}, \mathrm{CDCl}_{3}, \mathrm{ppm}\right): \delta 6.33$ (dt, $10.5 \mathrm{~Hz}, 16.8 \mathrm{~Hz}, 1 \mathrm{H}), 6.04$ (dd, $10.2 \mathrm{~Hz}, 15.3 \mathrm{~Hz}, 1 \mathrm{H}$ ), 5.59 (dd, $9.0 \mathrm{~Hz}, 15.3 \mathrm{~Hz}, 1 \mathrm{H}$ ), 5.50 (m, 1H), 5.11 (d, 16.8 Hz, 1H), 4.96 (d, 10.2 Hz, 1H), 4.25 (m, 1H), 3.31 (s, 3H), 2.93 (m, 1H), 1.99 $(\mathrm{m}, 1 \mathrm{H}), 1.86(\mathrm{~m}, 1 \mathrm{H}), 1.74(\mathrm{~m}, 3 \mathrm{H}), 1.56(\mathrm{~m}, 2 \mathrm{H}) ;{ }^{13} \mathrm{C}\left\{{ }^{1} \mathrm{H}\right\}$ NMR $\left(75 \mathrm{MHz}, \mathrm{CDCl}_{3}, \mathrm{ppm}\right): \delta$ 145.5, 138.8, 137.4, 130.9, 124.4, 115.2, 79.9, 56.2, 47.7, 31.2, 31.0, 15.4; IR (neat): 2960, 2927, 2815, 1647, 1446, 1195, 1091, 1003, 950, 897, 841; HR-MS calcd. for $\mathrm{C}_{12} \mathrm{H}_{19} \mathrm{O}(\mathrm{M}+\mathrm{H})$ : 179.1436, found 179.1432 .

COSY summary; the following cross peaks were observed: $\mathrm{H}(1)$ and $\mathrm{H}(2) ; \mathrm{H}(4)$ and $\mathrm{H}(5)$; $\mathrm{H}(5)$ and $\mathrm{H}(6) ; \mathrm{H}(6)$ and $\mathrm{H}(7) ; \mathrm{H}(7)$ and $\mathrm{H}(8) ; \mathrm{H}(8)$ and $\mathrm{H}(9) ; \mathrm{H}(9)$ and $\mathrm{H}(10) ; \mathrm{H}(10)$ and $\mathrm{H}(11)$. 
HMBC summary; the following cross peaks were observed: $\mathrm{H}(1)$ and $\mathrm{C}(2), \mathrm{C}(3) ; \mathrm{H}(2)$ and $\mathrm{C}(4), \mathrm{C}(7) ; \mathrm{H}(4)$ and $\mathrm{C}(5), \mathrm{C}(7), \mathrm{C}(\mathrm{MeO}) ; \mathrm{H}(5)$ and $\mathrm{C}(3), \mathrm{C}(4), \mathrm{C}(6), \mathrm{C}(7) ; \mathrm{H}(6)$ and $\mathrm{C}(3), \mathrm{C}(4)$, C(5), C(7), C(8); H(8) and C(7), C(10); H(9) and C(7), C(10), C(11); H(10) and C(8), C(9); H(11) and $\mathrm{C}(9)$.

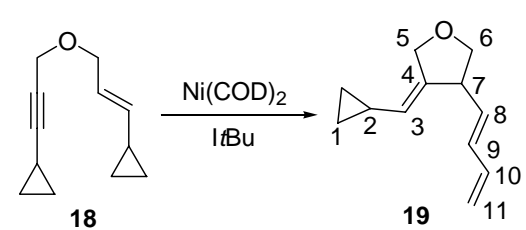

Rearrangement of [3-(3-cyclopropyl-2-pentenyloxy)-1propynyl]-cyclopropane (18) catalyzed by $\mathrm{Ni}(\mathrm{COD})_{2} / \mathrm{ItBu}$. The general procedure was used with [3-(3-cyclopropyl-2pentenyloxy)-1-propynyl]-cyclopropane (18, $100 \mathrm{mg}, \quad 0.57$ mmol), $\mathrm{Ni}(\mathrm{COD})_{2}$ (8 $\left.\mathrm{mg}, 0.03 \mathrm{mmol}\right), \mathrm{ItBu}(5.4 \mathrm{mg}, 0.03$ $\mathrm{mmol})$, and $0.5 \mathrm{~mL}$ of toluene. The reaction mixture was purified by column chromatography on silica gel $\left(10 \% \mathrm{Et}_{2} \mathrm{O} /\right.$ pentanes) to afford 3-(1,3butadienyl)- 4-(2-cyclopropylpropylidene)-tetrahydrofuran (19, $65 \mathrm{mg}, 65 \%)$ as a colorless oil. ${ }^{1} \mathrm{H}$ NMR (300 MHz, CDCl 3 , ppm): $\delta 6.62$ (dt, 10.5 Hz, 16.8 Hz, 1H), 6.15 (t, 10.5 Hz, 1H), 5.22 (m, 3H), $4.58(\mathrm{~m}, 2 \mathrm{H}), 4.46(\mathrm{~m}, 1 \mathrm{H}), 4.10(\mathrm{t}, 8.4 \mathrm{~Hz}, 1 \mathrm{H}), 3.76(\mathrm{~m}, 1 \mathrm{H}), 3.41(\mathrm{t}, 8.4 \mathrm{~Hz}, 1 \mathrm{H}), 1.17(\mathrm{~m}$, $1 \mathrm{H}), 0.73(\mathrm{~m}, 4 \mathrm{H}), 0.34(\mathrm{~m}, 2 \mathrm{H}) ;{ }^{13} \mathrm{C}\left\{{ }^{1} \mathrm{H}\right\}$ NMR (75 MHz, $\left.\mathrm{CDCl}_{3}, \mathrm{ppm}\right): \delta$ 139.8, 132.2, 131.5, 130.3, 125.3, 118.7, 73.7, 69.8, 43.3, 11.7, 6.99, 6.86; IR (neat): 3083, 3006, 2843, 1643, 1433, 1072, 1000, 961, 907, 807; HR-MS calcd. for $\mathrm{C}_{12} \mathrm{H}_{17} \mathrm{O}(\mathrm{M}+\mathrm{H}): 177.1279$, found 177.1279.

COSY summary; the following cross peaks were observed: $\mathrm{H}(1)$ and $\mathrm{H}(2) ; \mathrm{H}(2)$ and $\mathrm{H}(3)$; $\mathrm{H}(6)$ and $\mathrm{H}(7) ; \mathrm{H}(7)$ and $\mathrm{H}(8) ; \mathrm{H}(8)$ and $\mathrm{H}(9) ; \mathrm{H}(9)$ and $\mathrm{H}(10) ; \mathrm{H}(10)$ and $\mathrm{H}(11)$.

HMBC summary; the following cross peaks were observed: $\mathrm{H}(1)$ and $\mathrm{C}(2), \mathrm{C}(3) ; \mathrm{H}(3)$ and $\mathrm{C}(4), \mathrm{C}(5) ; \mathrm{H}(5)$ and $\mathrm{C}(3), \mathrm{C}(4), \mathrm{C}(6), \mathrm{C}(7) ; \mathrm{H}(6)$ and $\mathrm{C}(4), \mathrm{C}(5), \mathrm{C}(7), \mathrm{C}(8) ; \mathrm{H}(7)$ and $\mathrm{C}(3), \mathrm{C}(4)$, $\mathrm{C}(6), \mathrm{C}(8), \mathrm{C}(9) ; \mathrm{H}(8)$ and $\mathrm{C}(6), \mathrm{C}(7) ; \mathrm{H}(9)$ and $\mathrm{C}(7), \mathrm{C}(10), \mathrm{C}(11) ; \mathrm{H}(10)$ and $\mathrm{C}(8), \mathrm{C}(9) ; \mathrm{H}(11)$ and $\mathrm{C}(9)$.

\section{References}

(1) Böhm, V. P. W., Gstöttmayr, C. W. K., Weskamp, T.; Herrmann, W. A. Angew. Chem. Int. Ed. 2001, 40, 3387-3389.

(2) (a) Wang, B.; Cao, P.; Zhang, X. Tetrahedron Lett. 2000, 41, 8041-8044.

(b) Wender, P. A.; Takahashi, H.; Witulski, B. J. Am. Chem. Soc. 1995, 117, 4720-4721.

(c) Wender, P. A.; Williams, T. J. Angew. Chem. Int. Ed. 2002, 41, 4550-4553.

(d) Chatani, N.; Inoue, H.; Morimoto, T.; Muto, T.; Murai, S. J. Org. Chem. 2001, 66, 44334436.

(3) MacInnes, I.; Walton, J. C. J. Chem. Soc., Perkin Trans. 2 1987, 8, 1077-1082.

(4) An equilibrium exists between $\mathrm{Ni}(\mathrm{COD})_{2} / \mathrm{IPr}$ and $\mathrm{Ni}(\mathrm{IPr})_{2} / \mathrm{COD}$, see: Louie, J.; Gibby, J. E.;

Farnworth, M. V.; Tekavec, T. N. J. Am. Chem. Soc. 2002, 124, 15188-15189. 


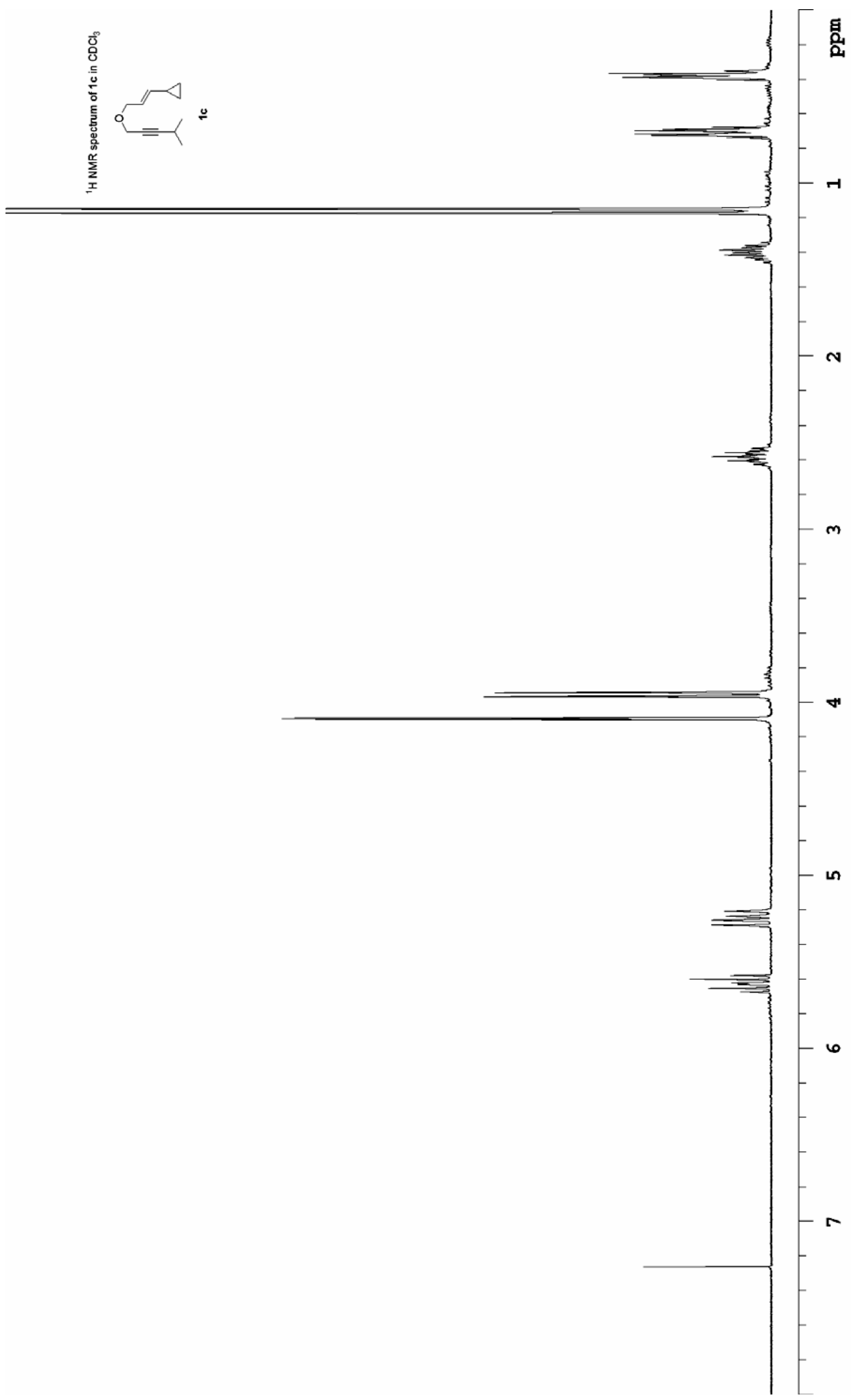



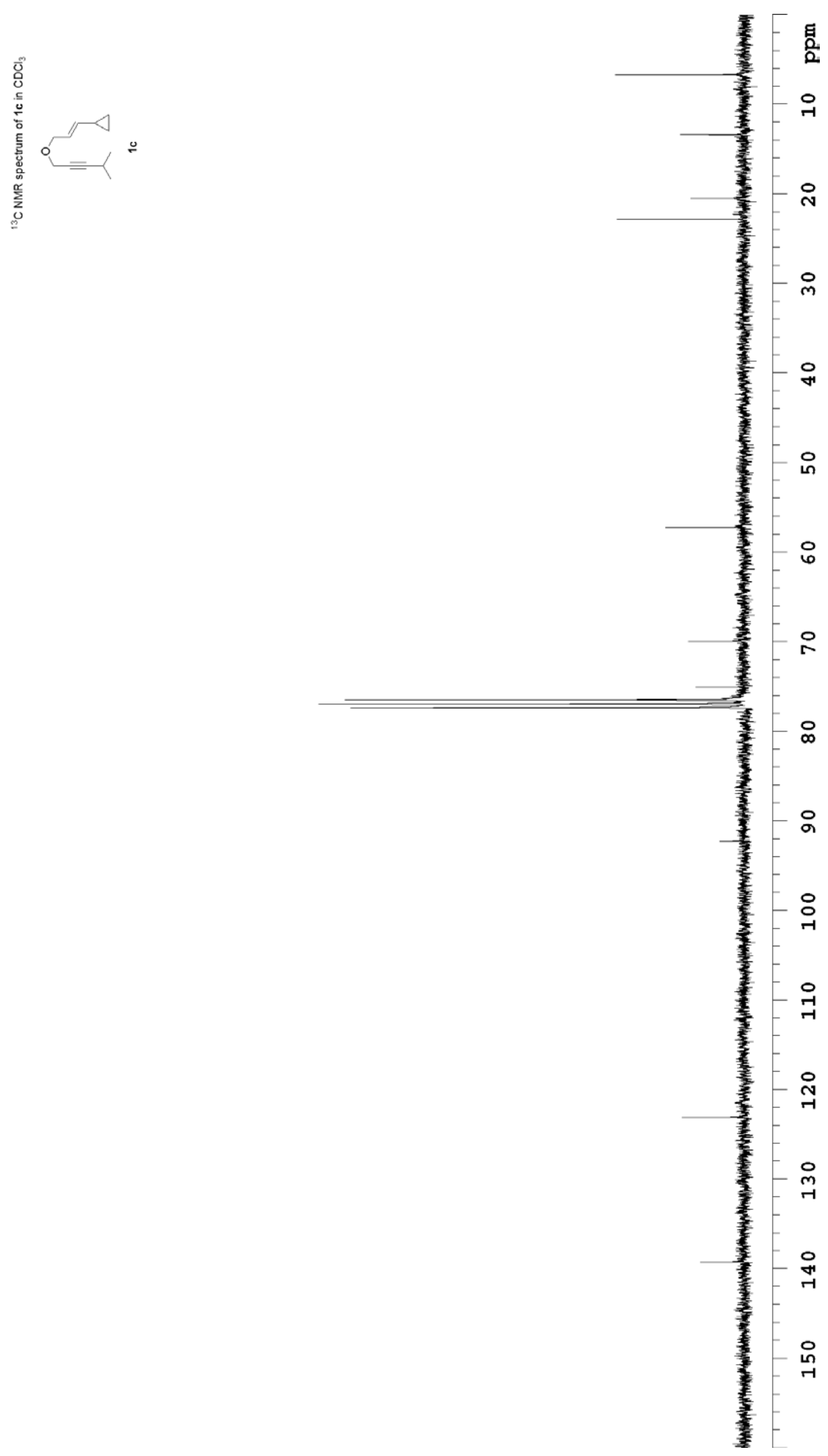


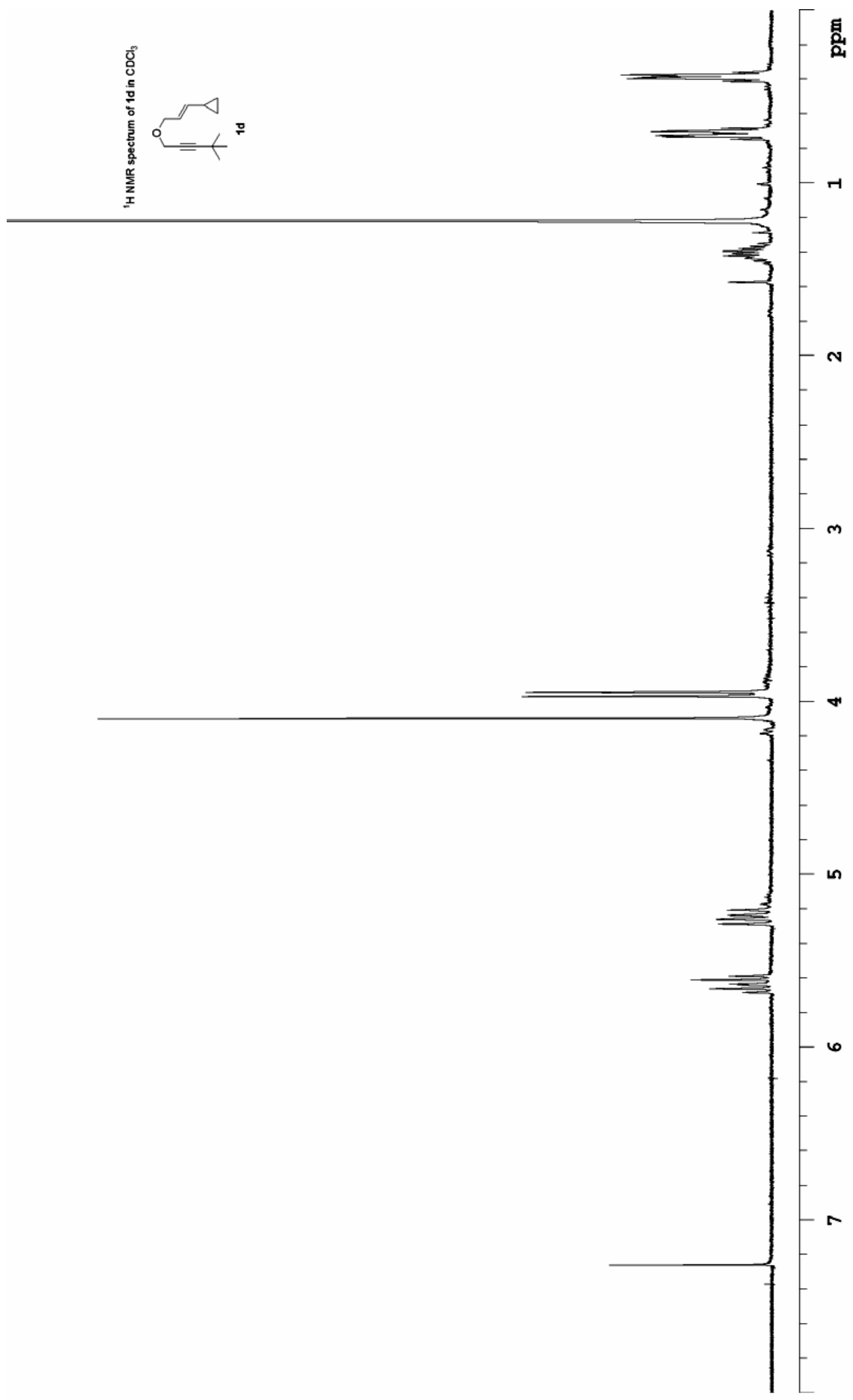



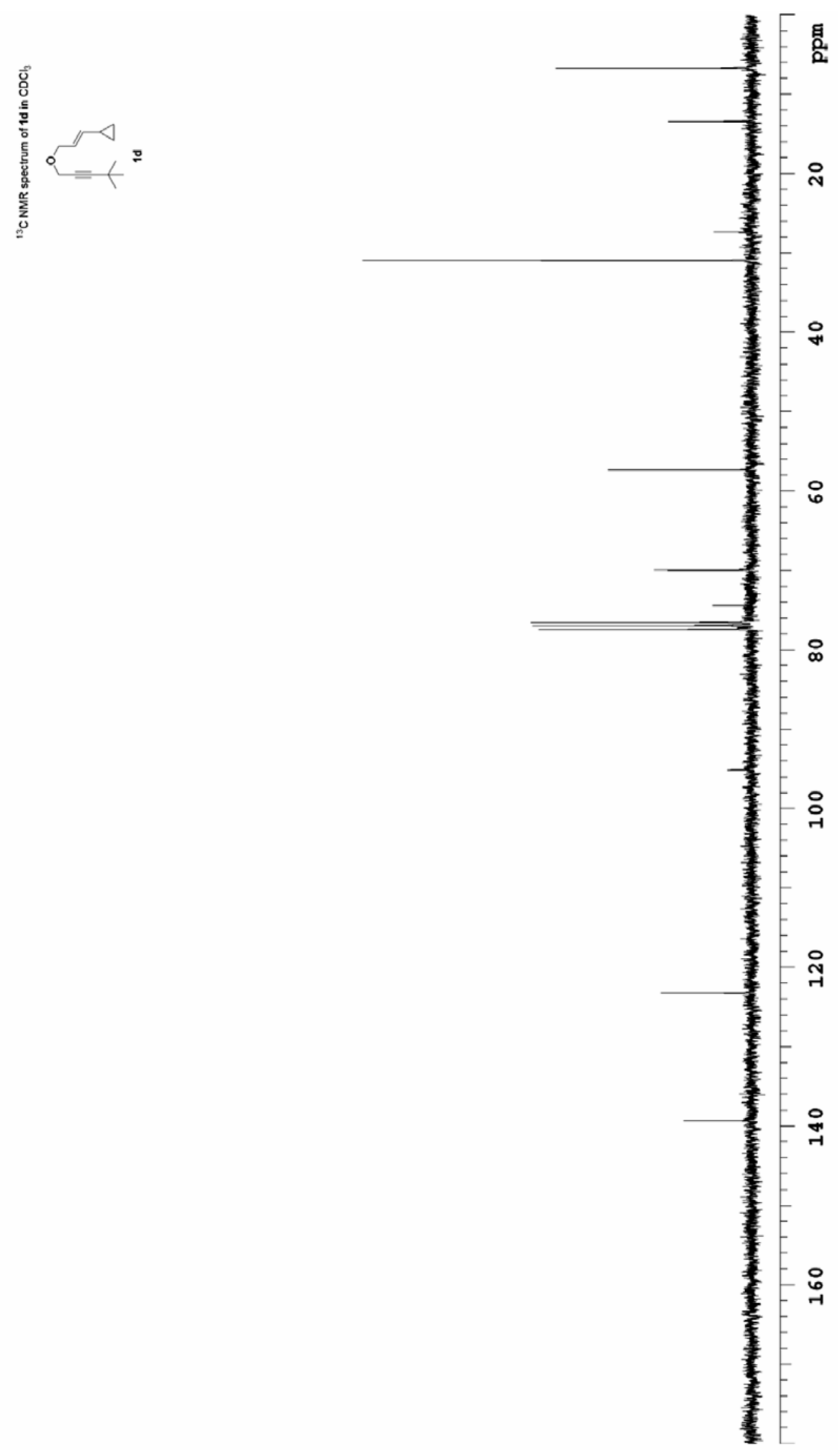


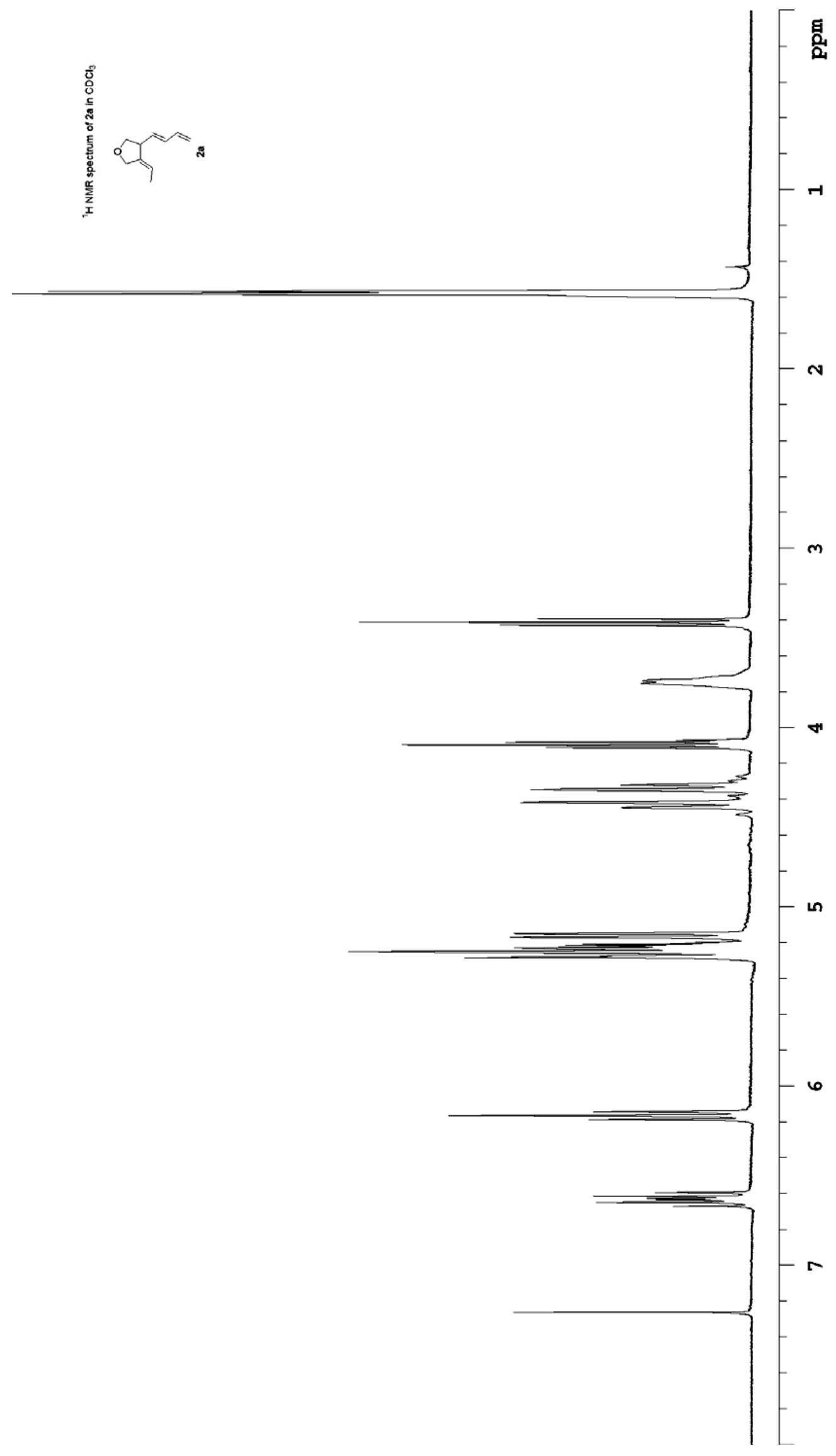




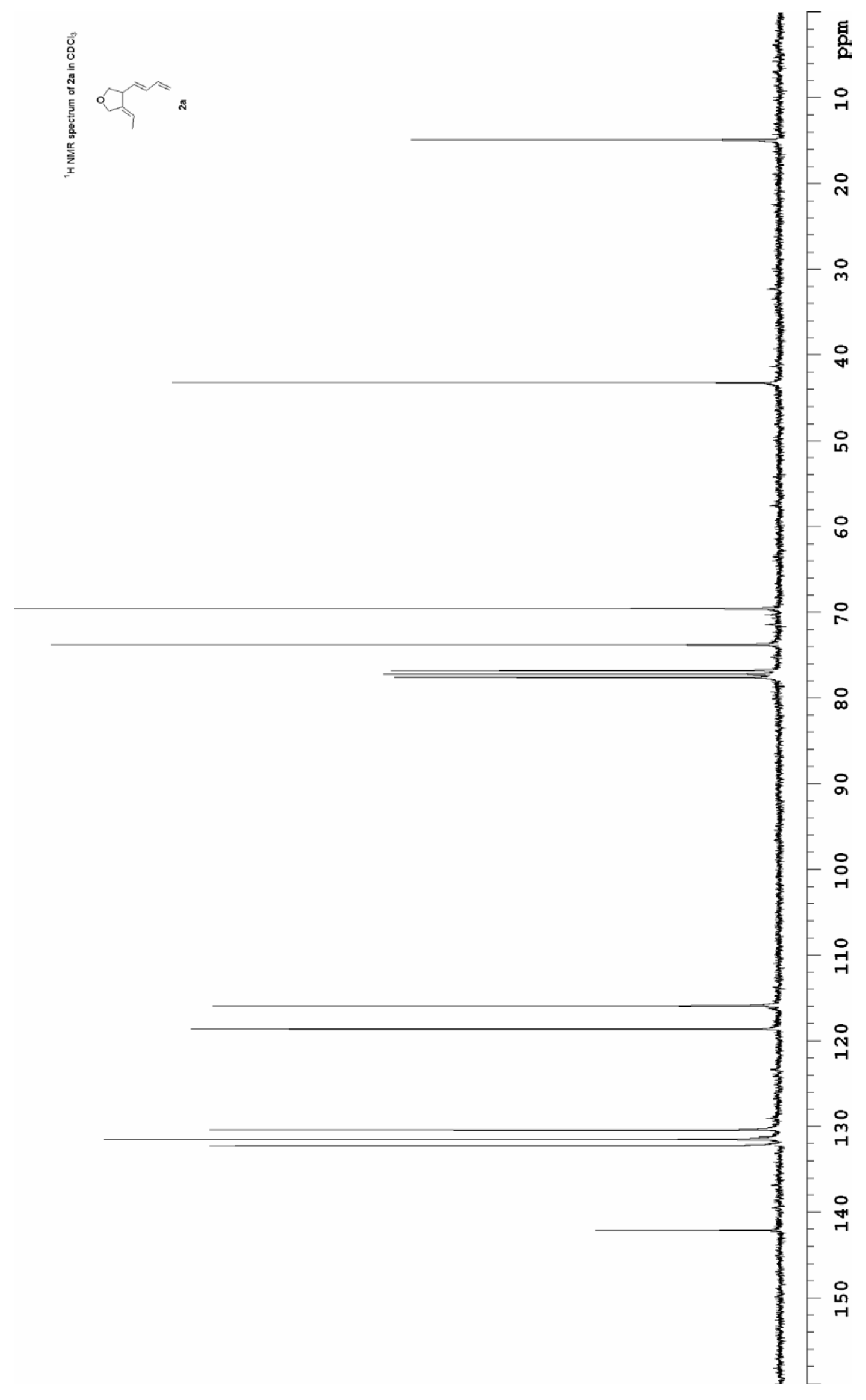



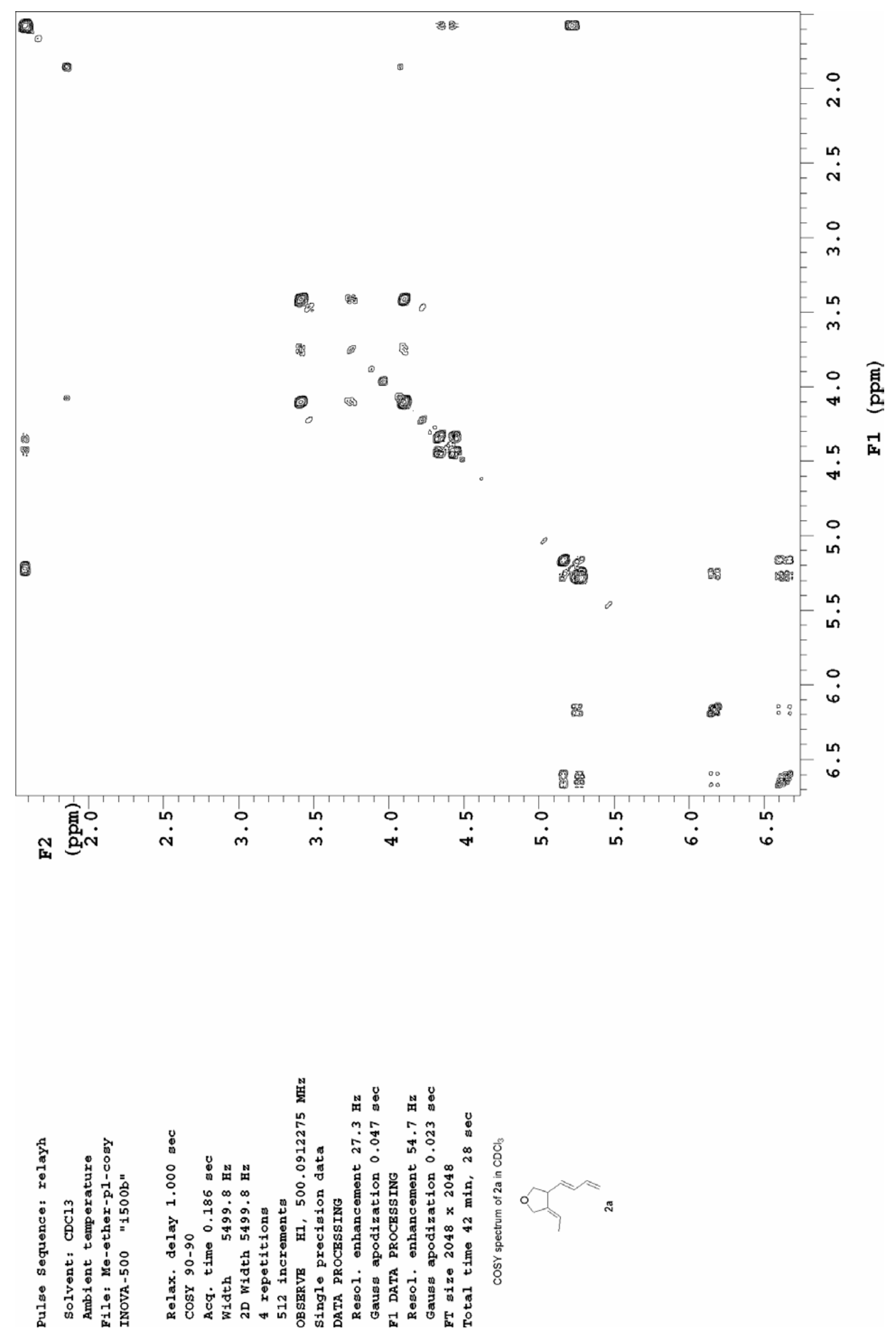

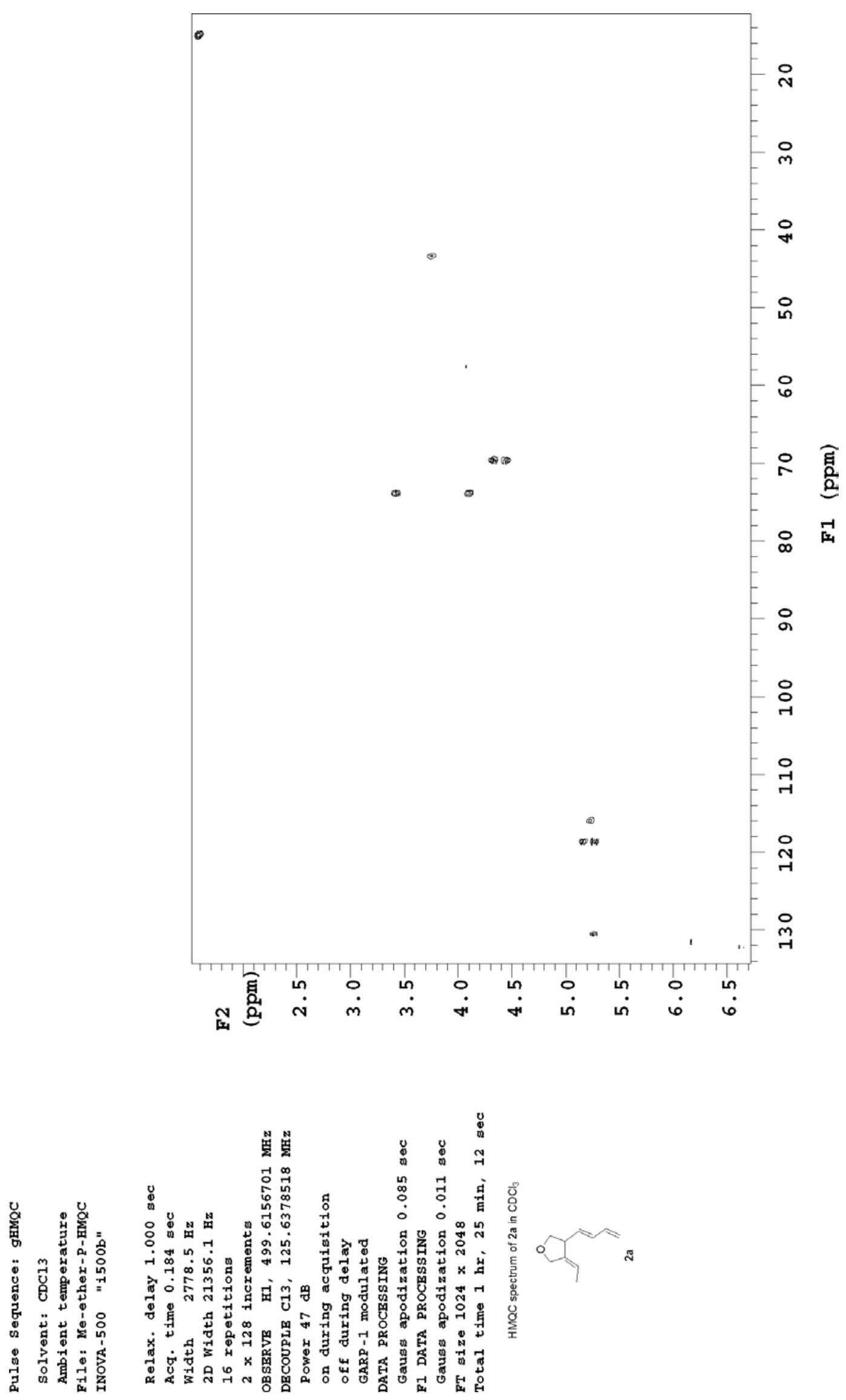

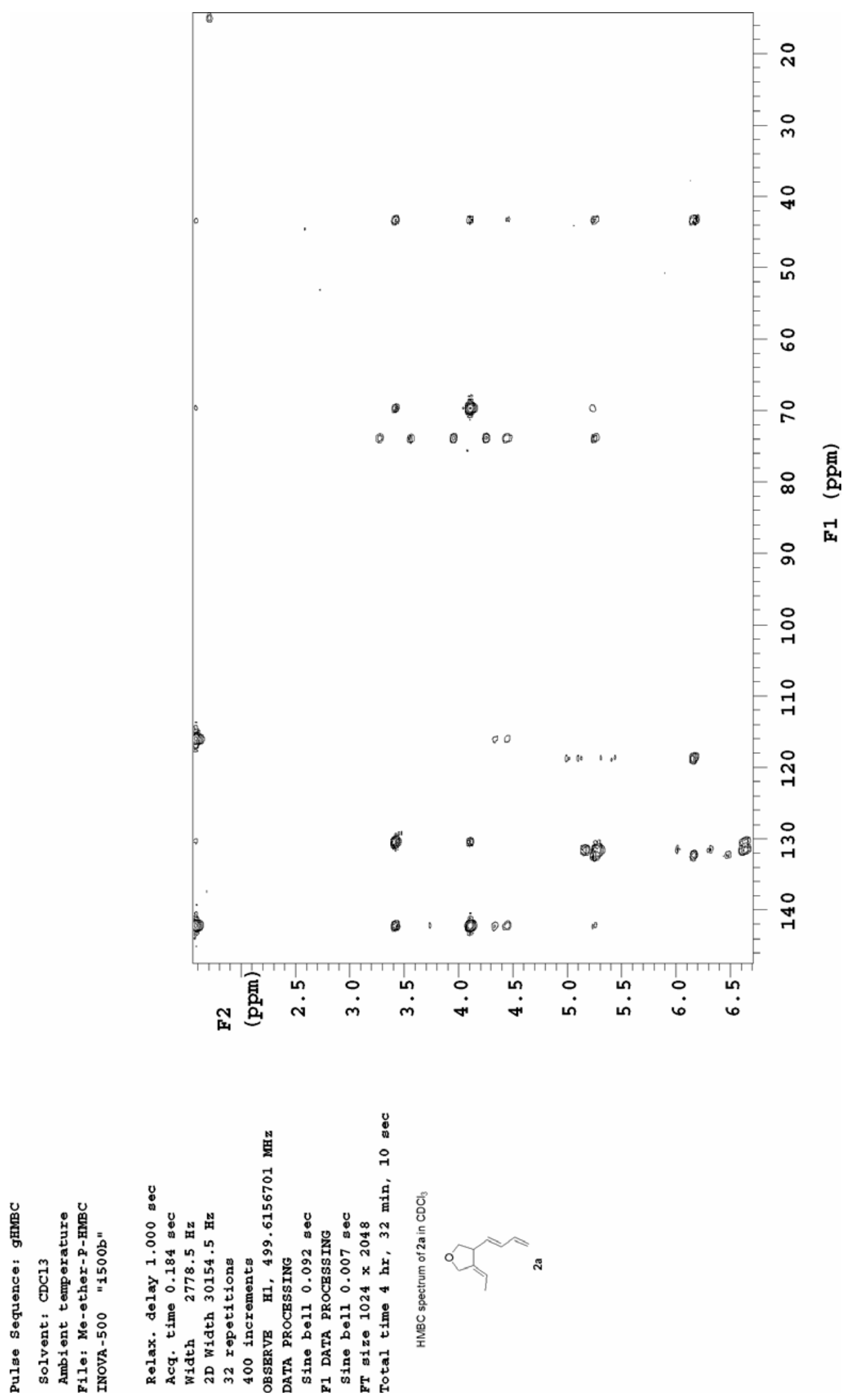


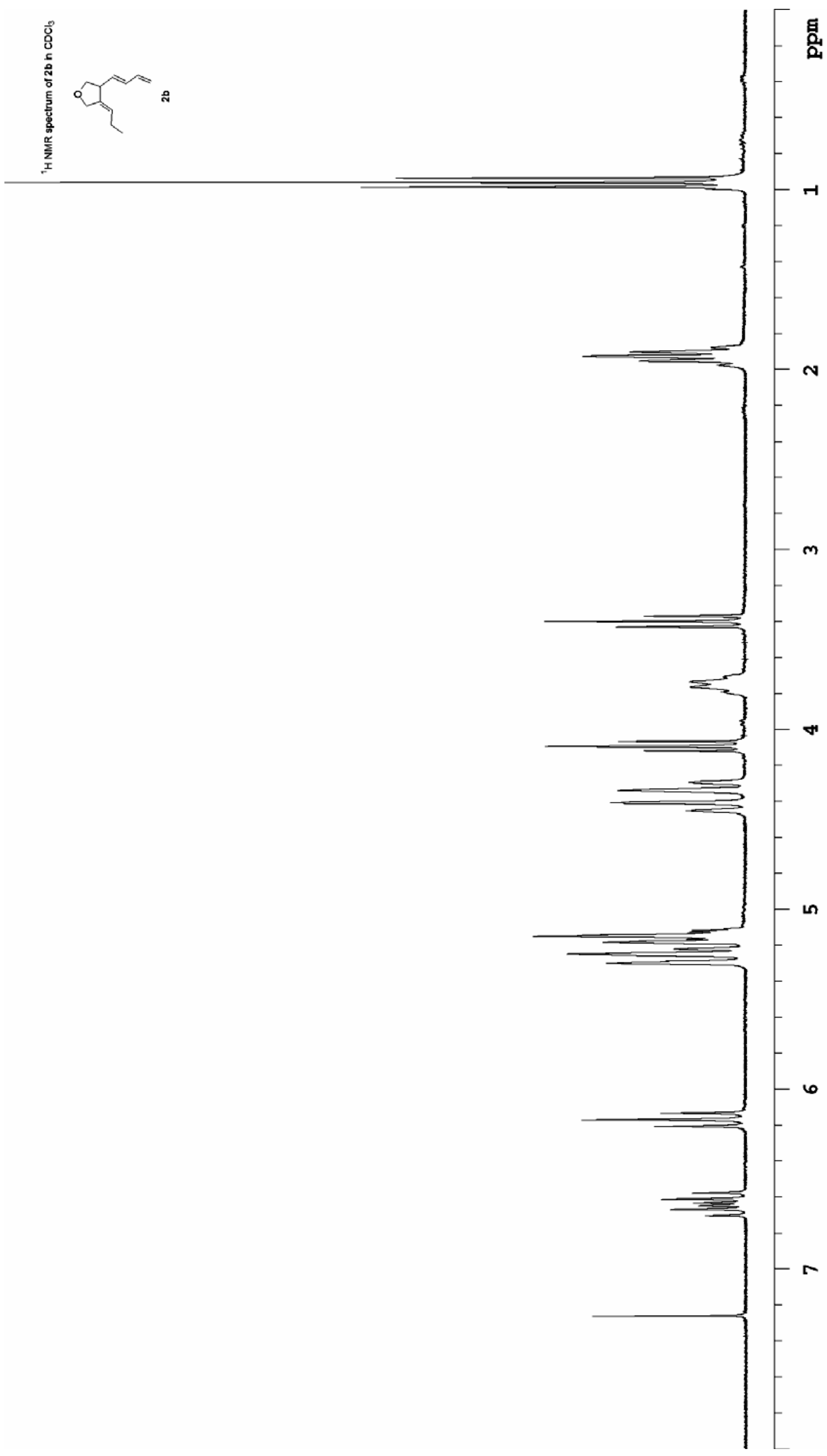




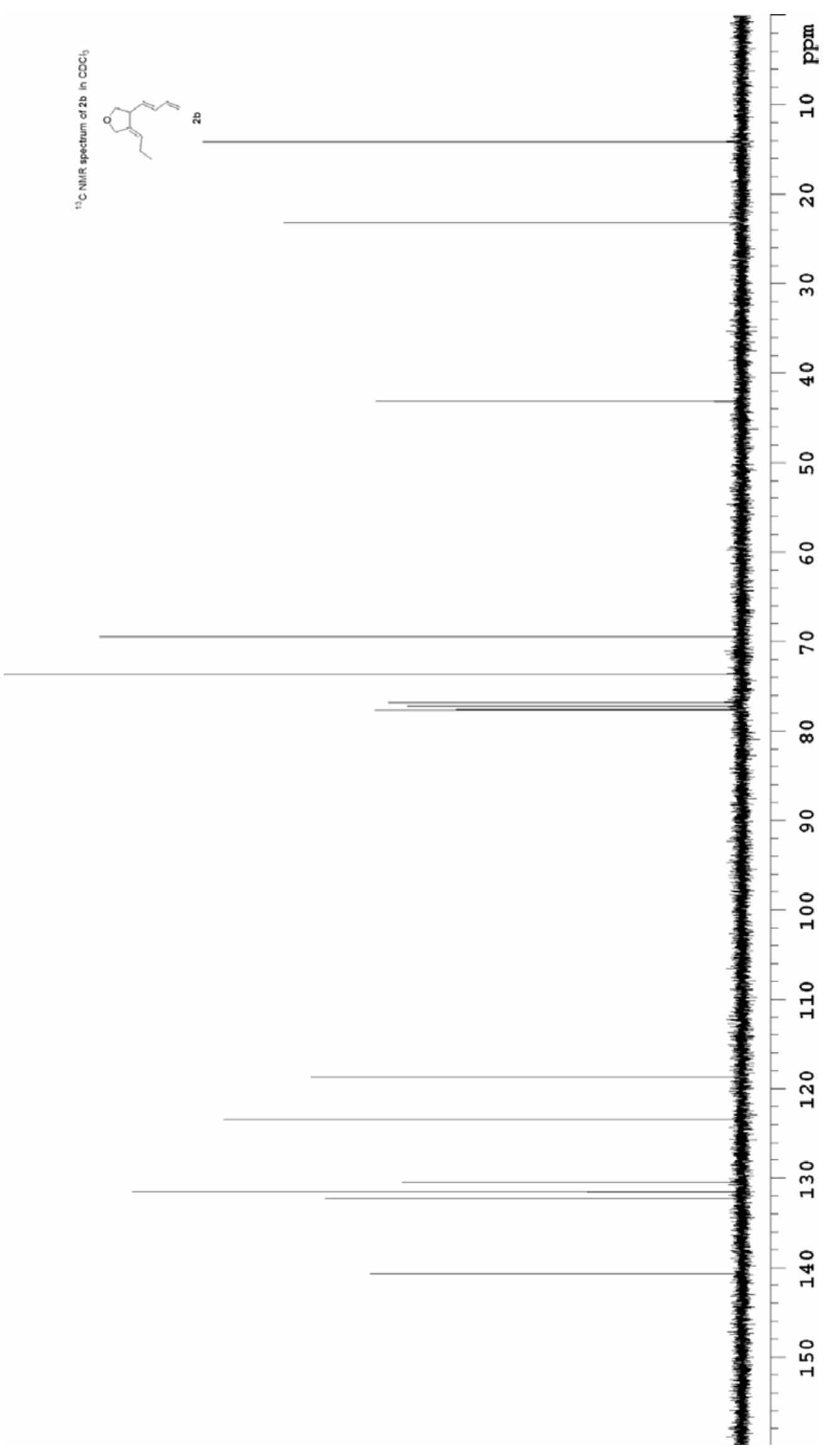



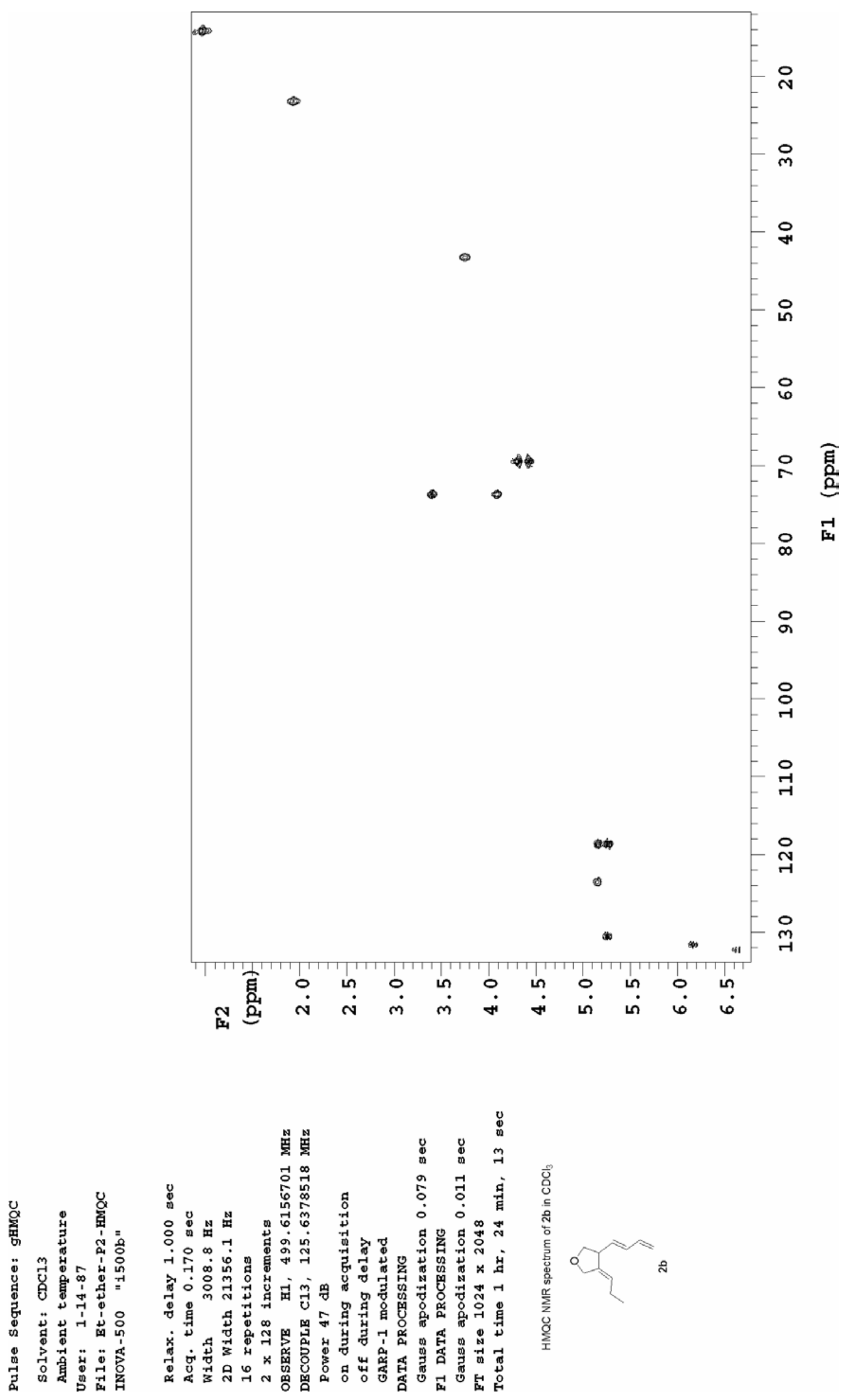

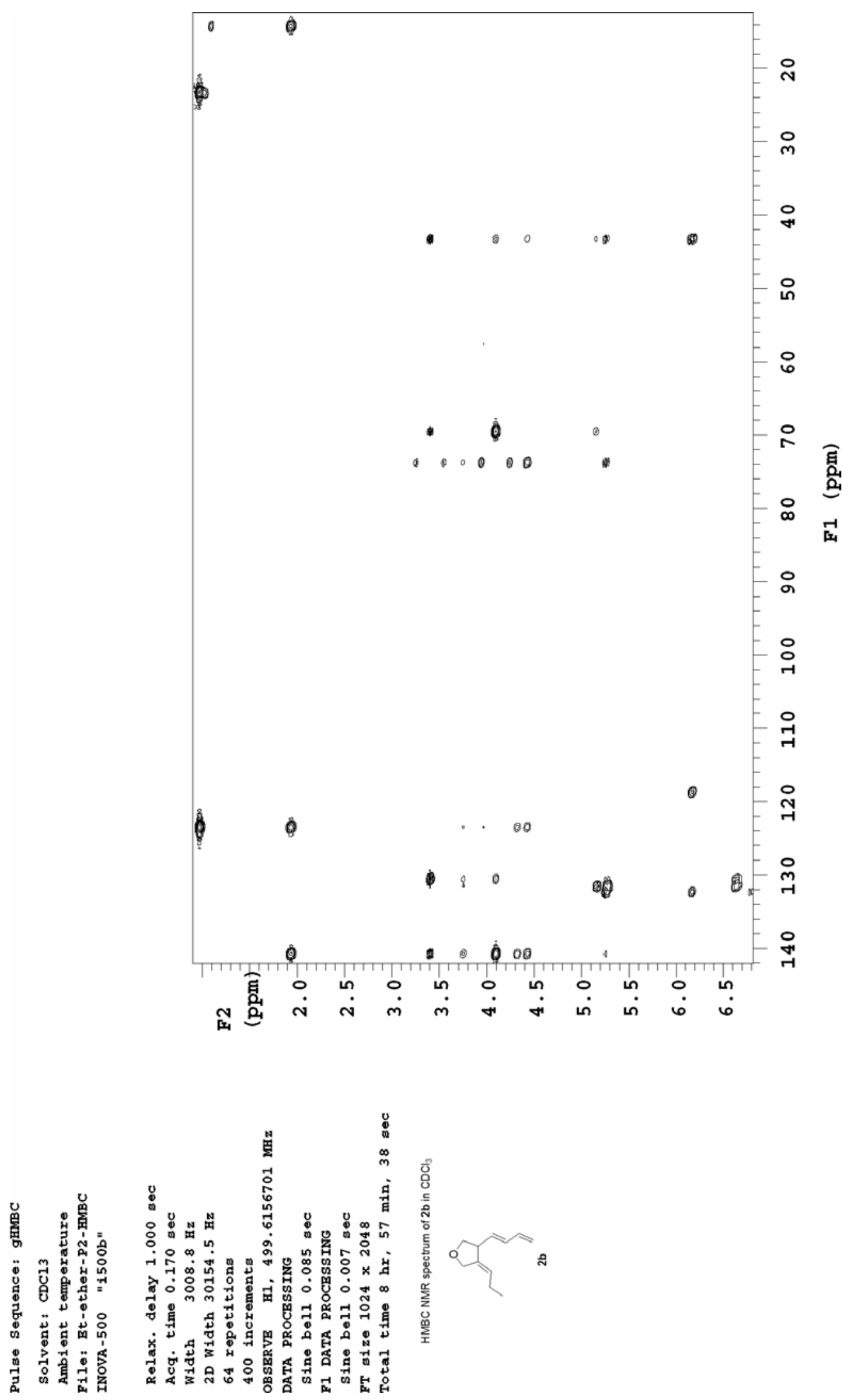


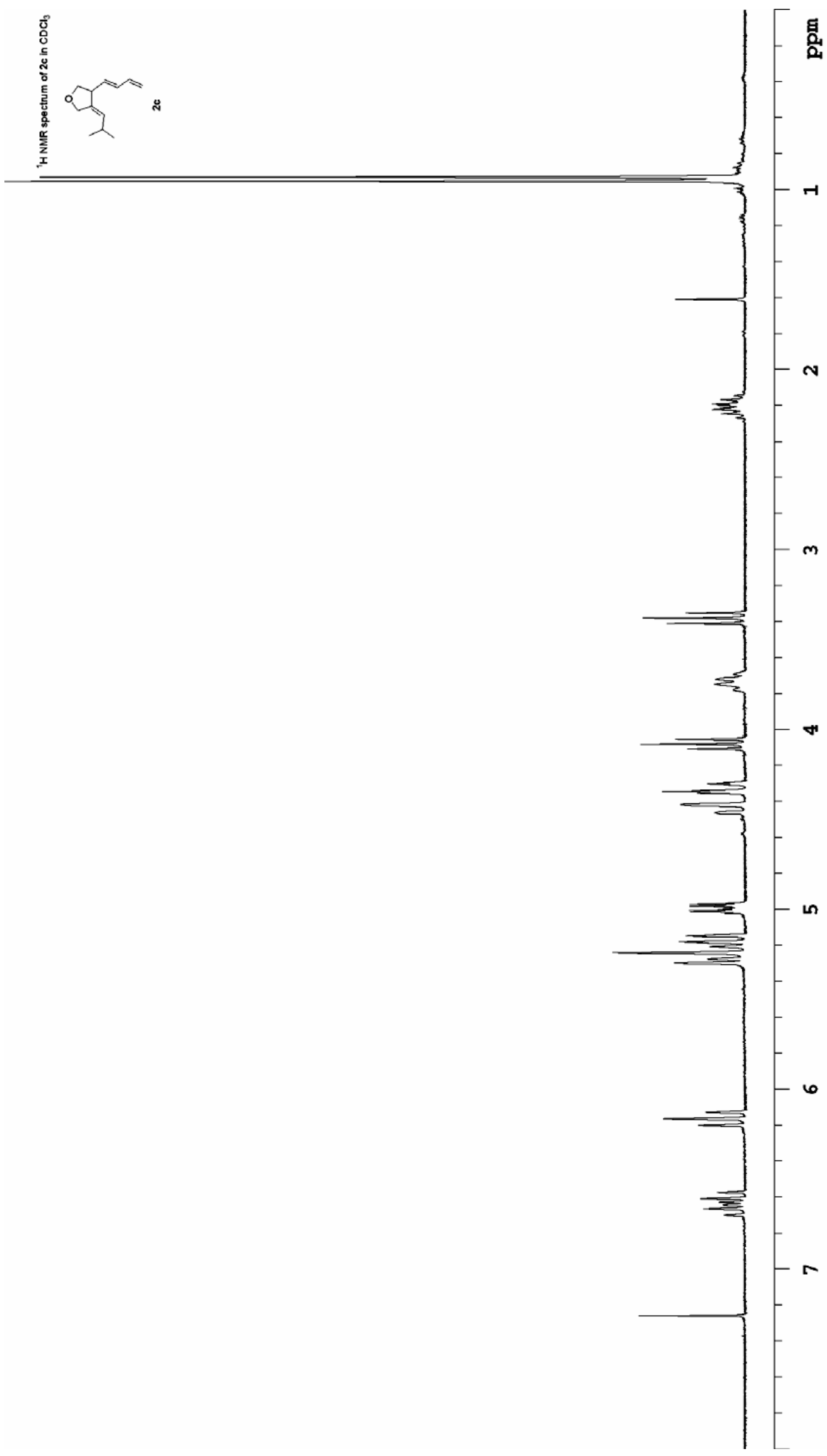



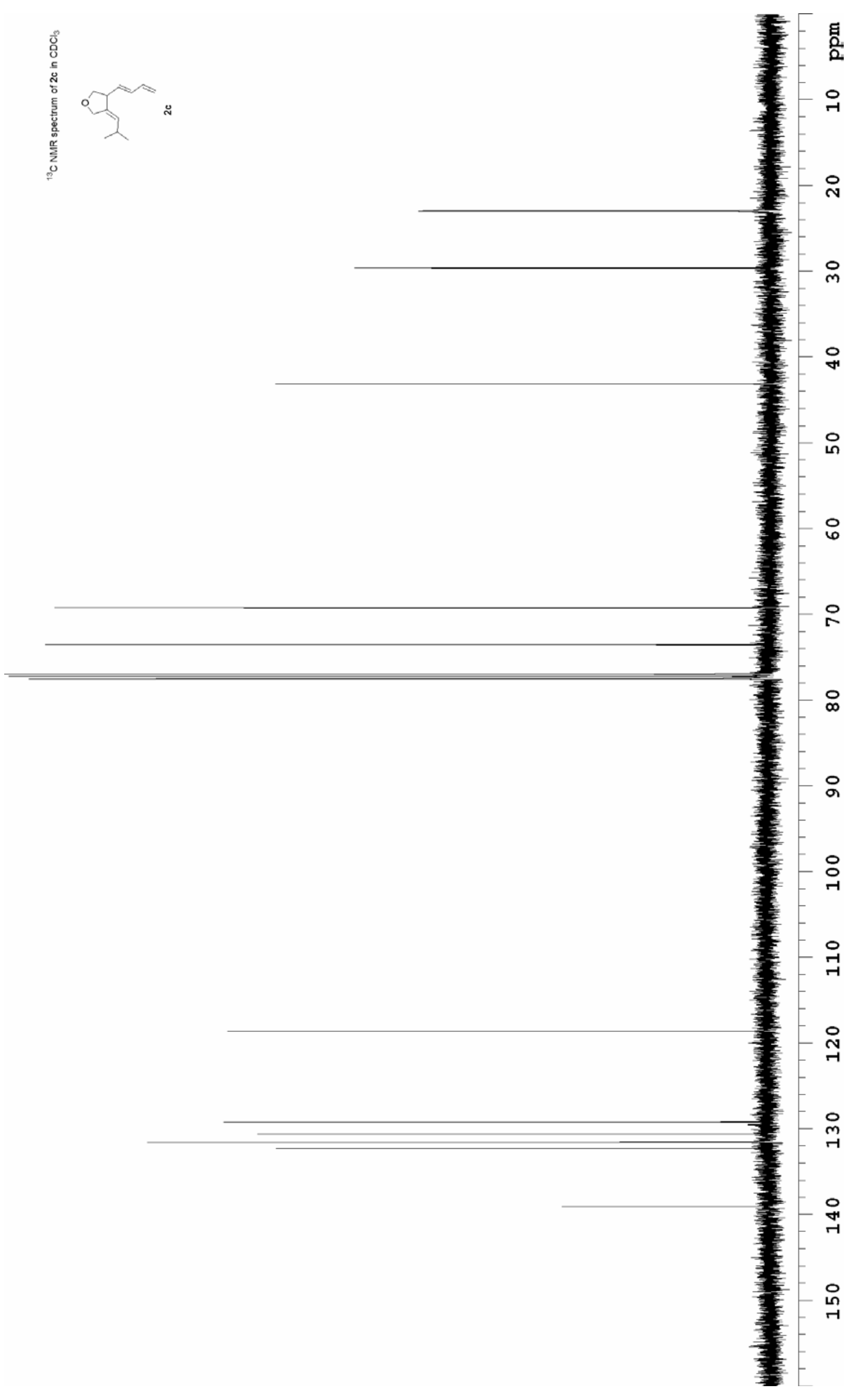

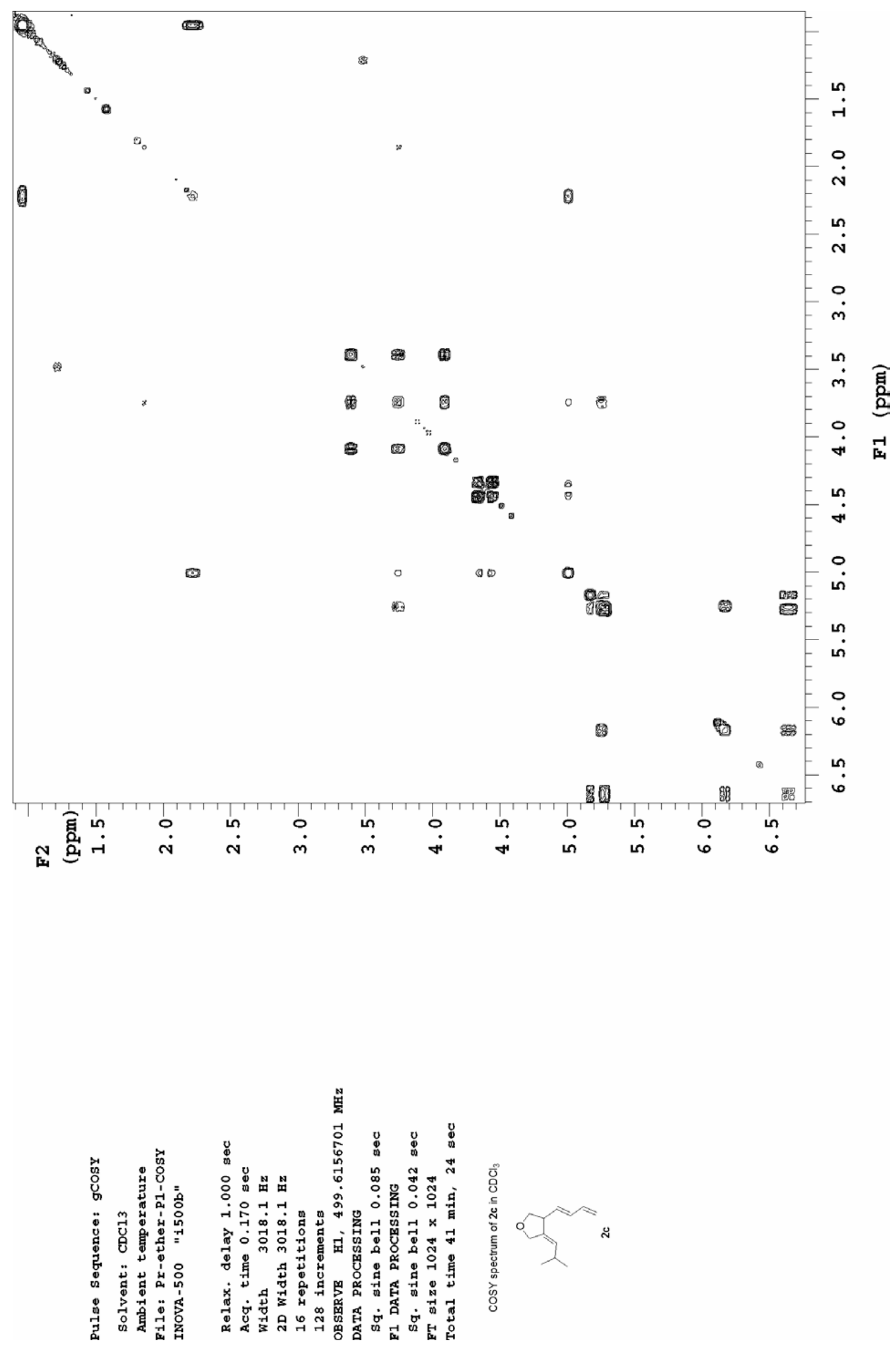

S25 

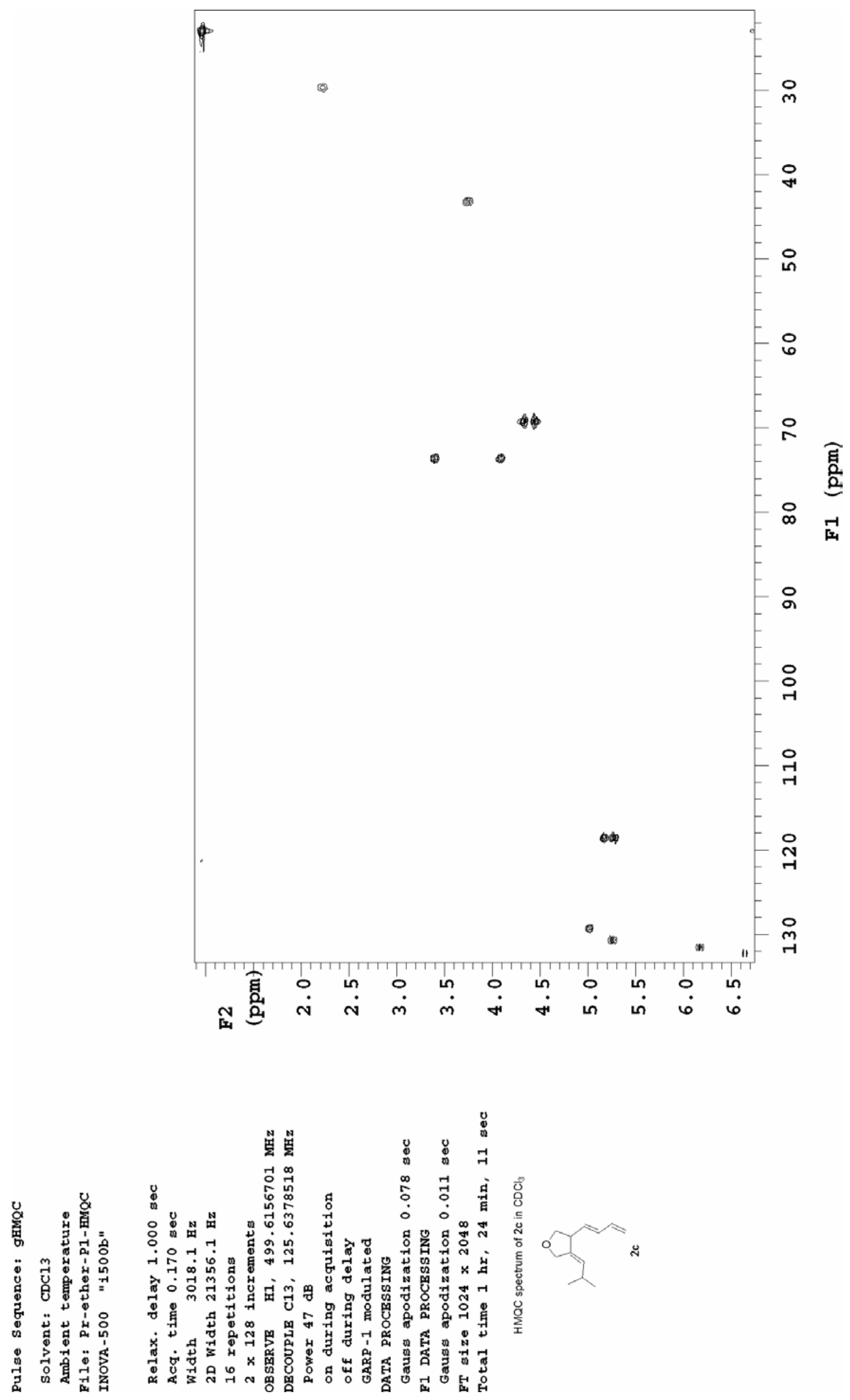

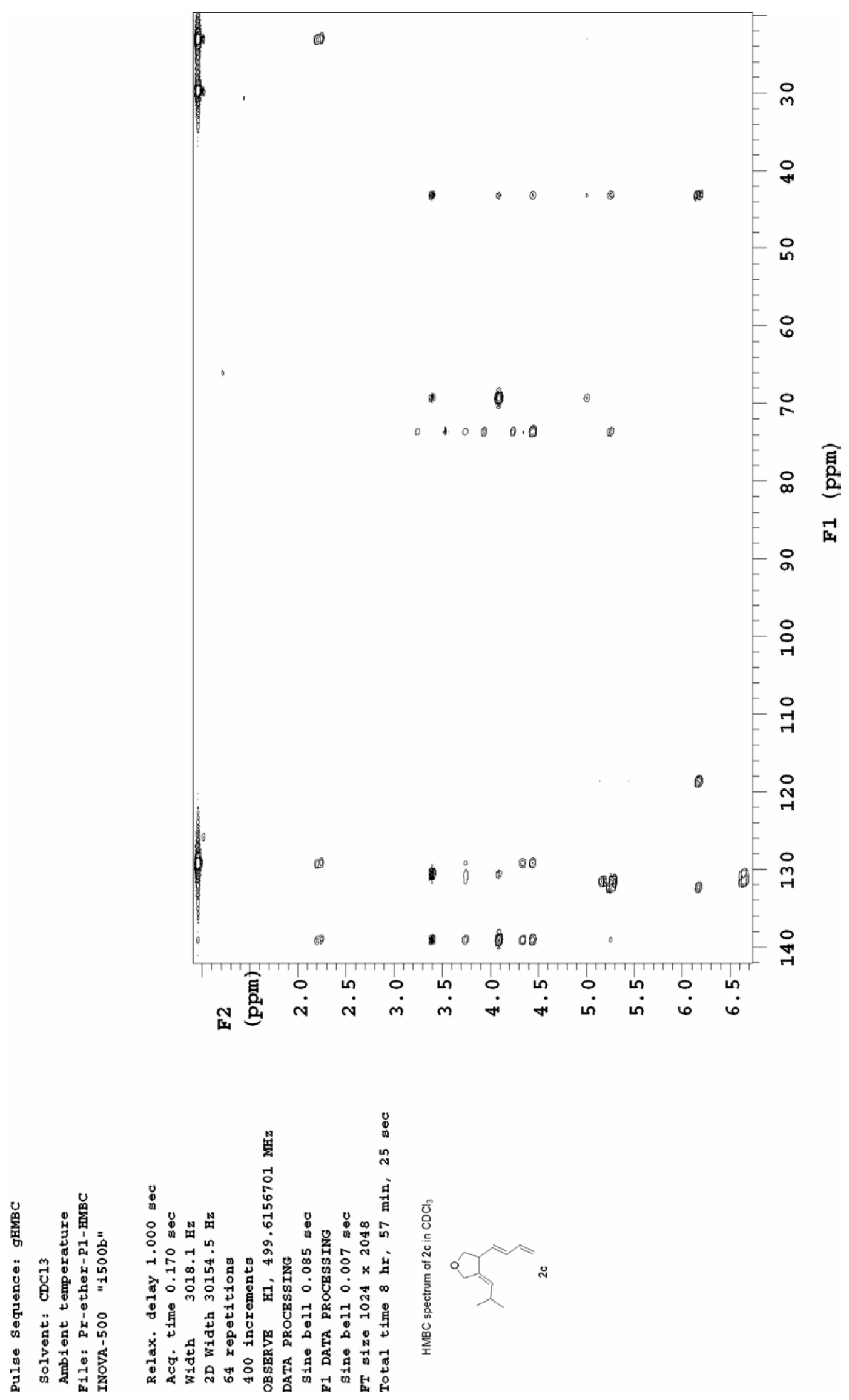


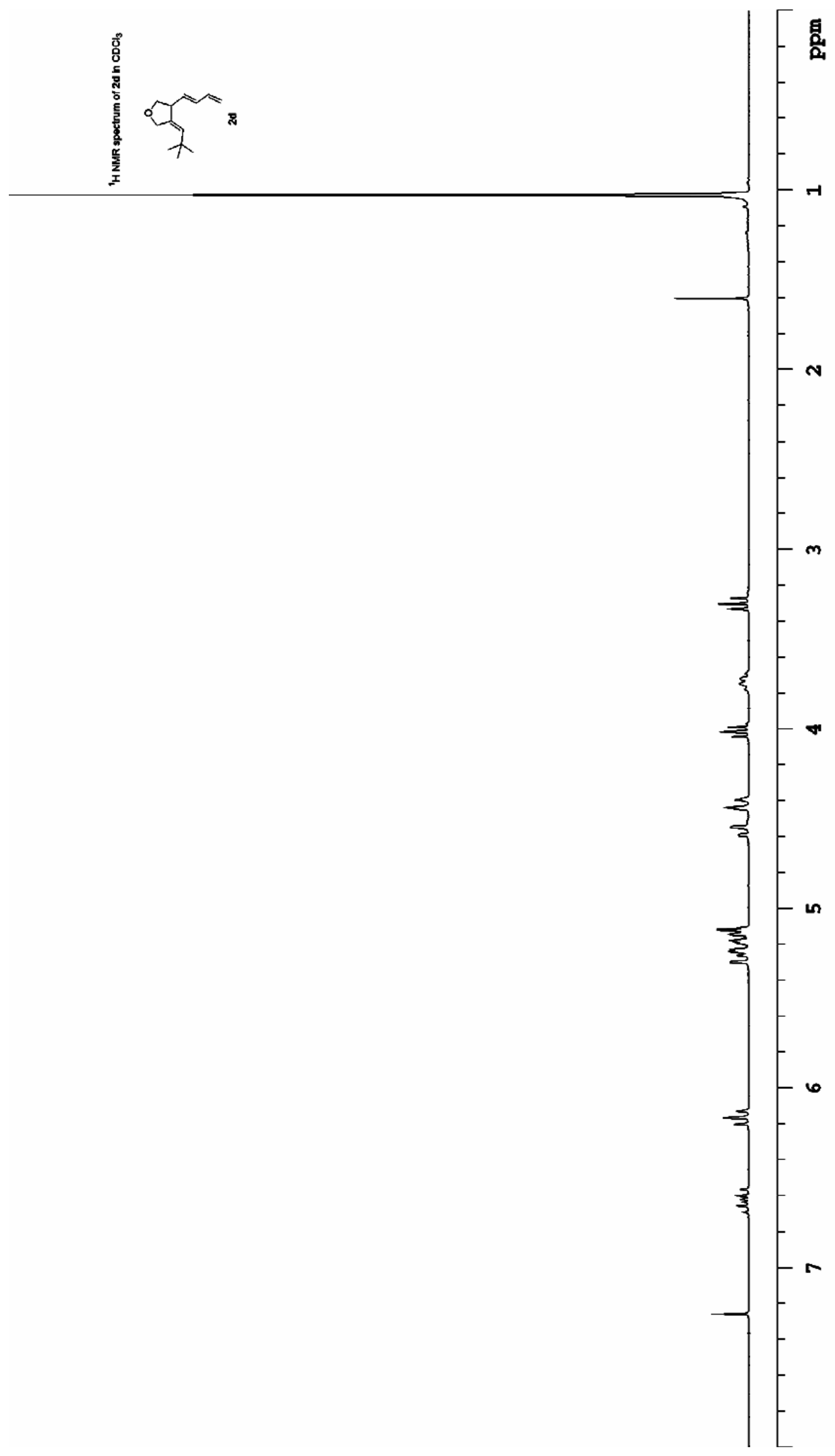

S28 

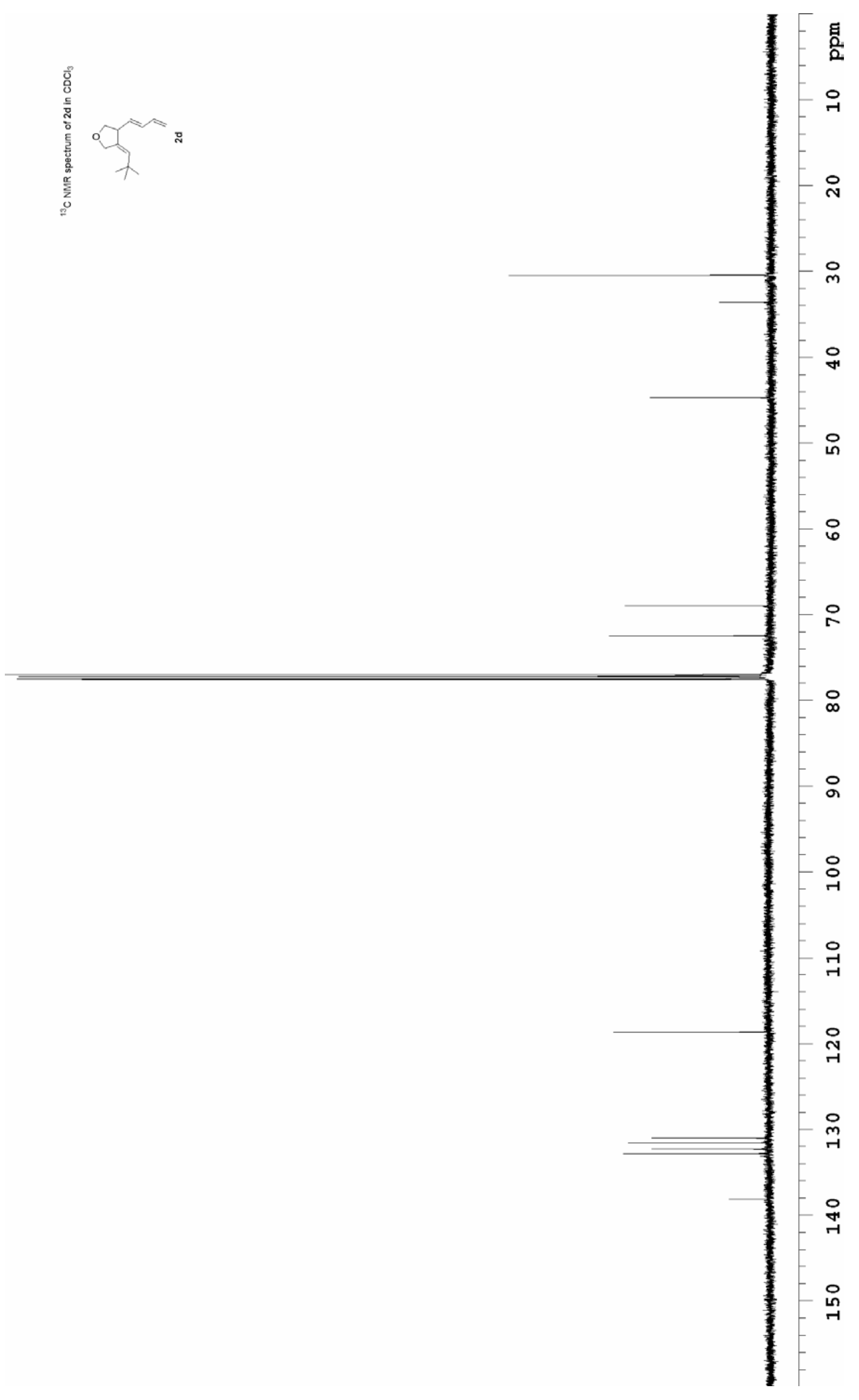

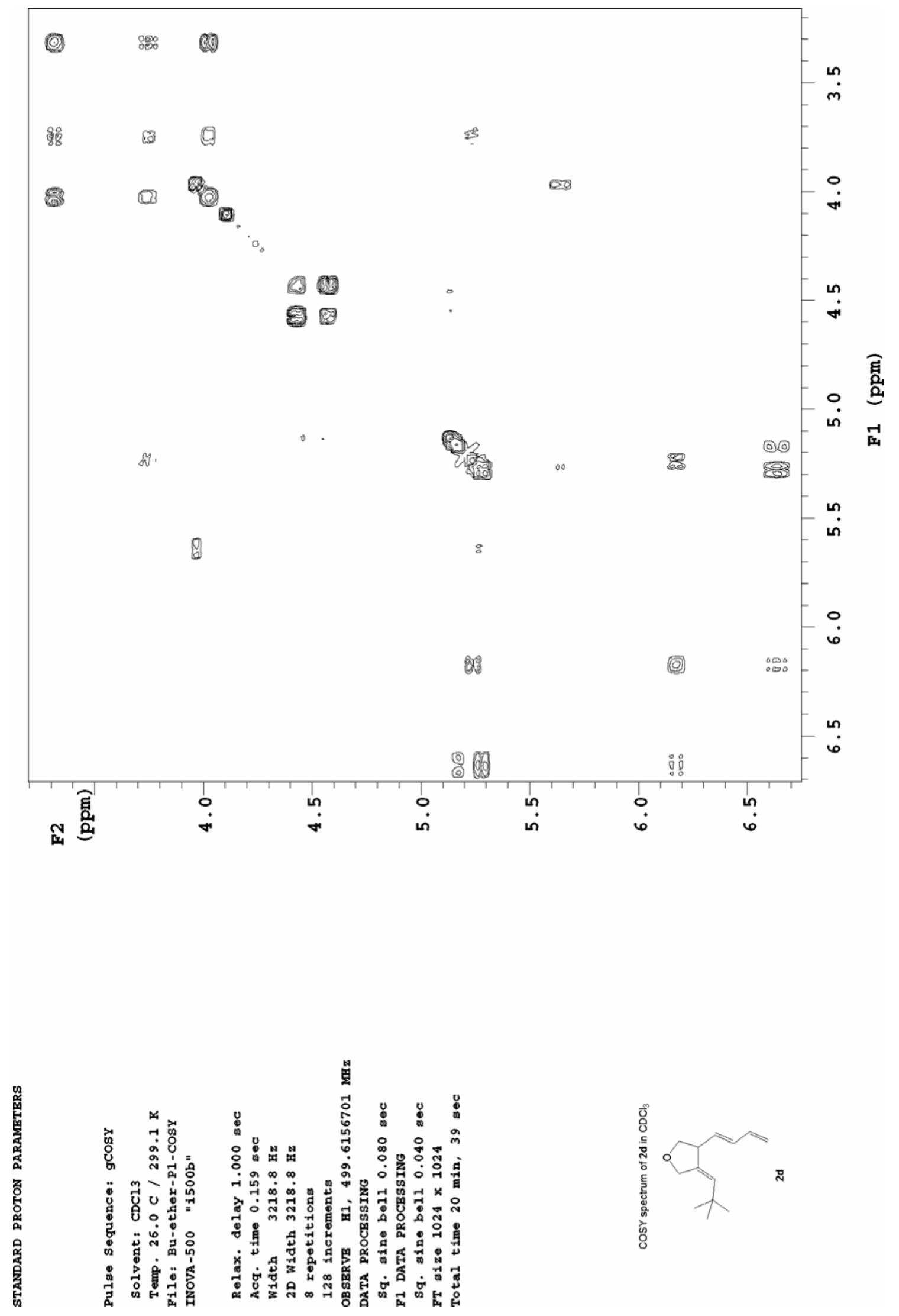

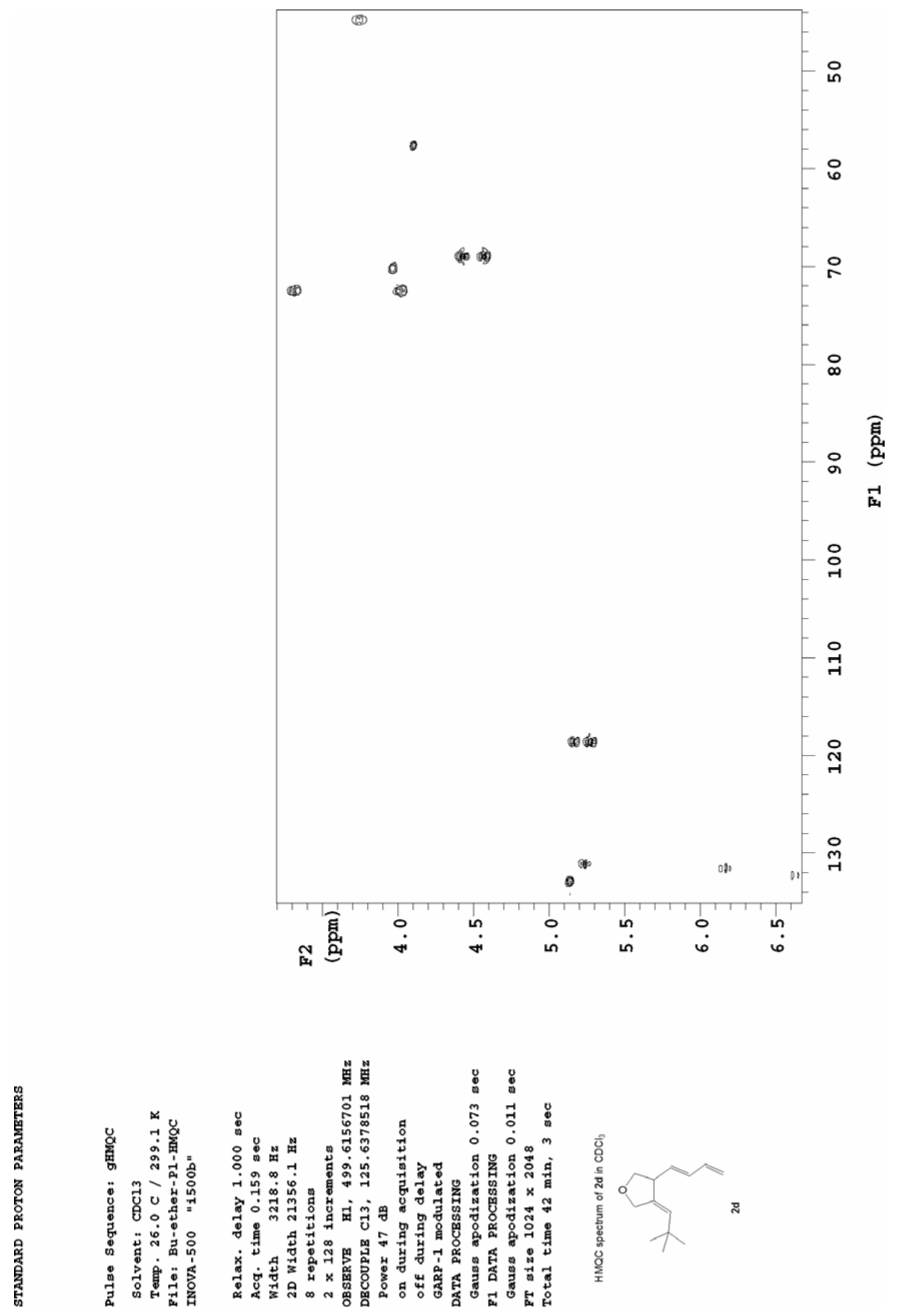

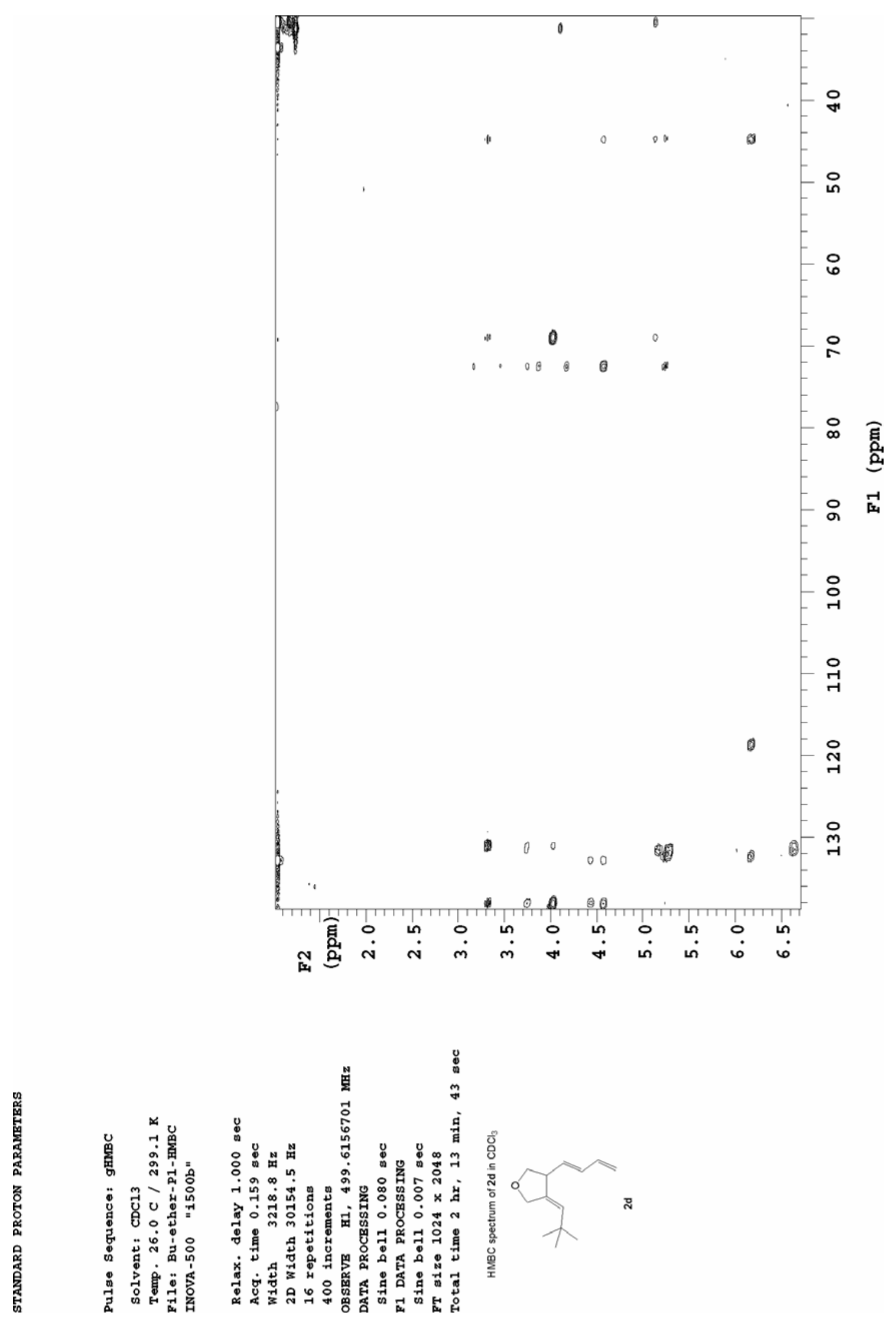


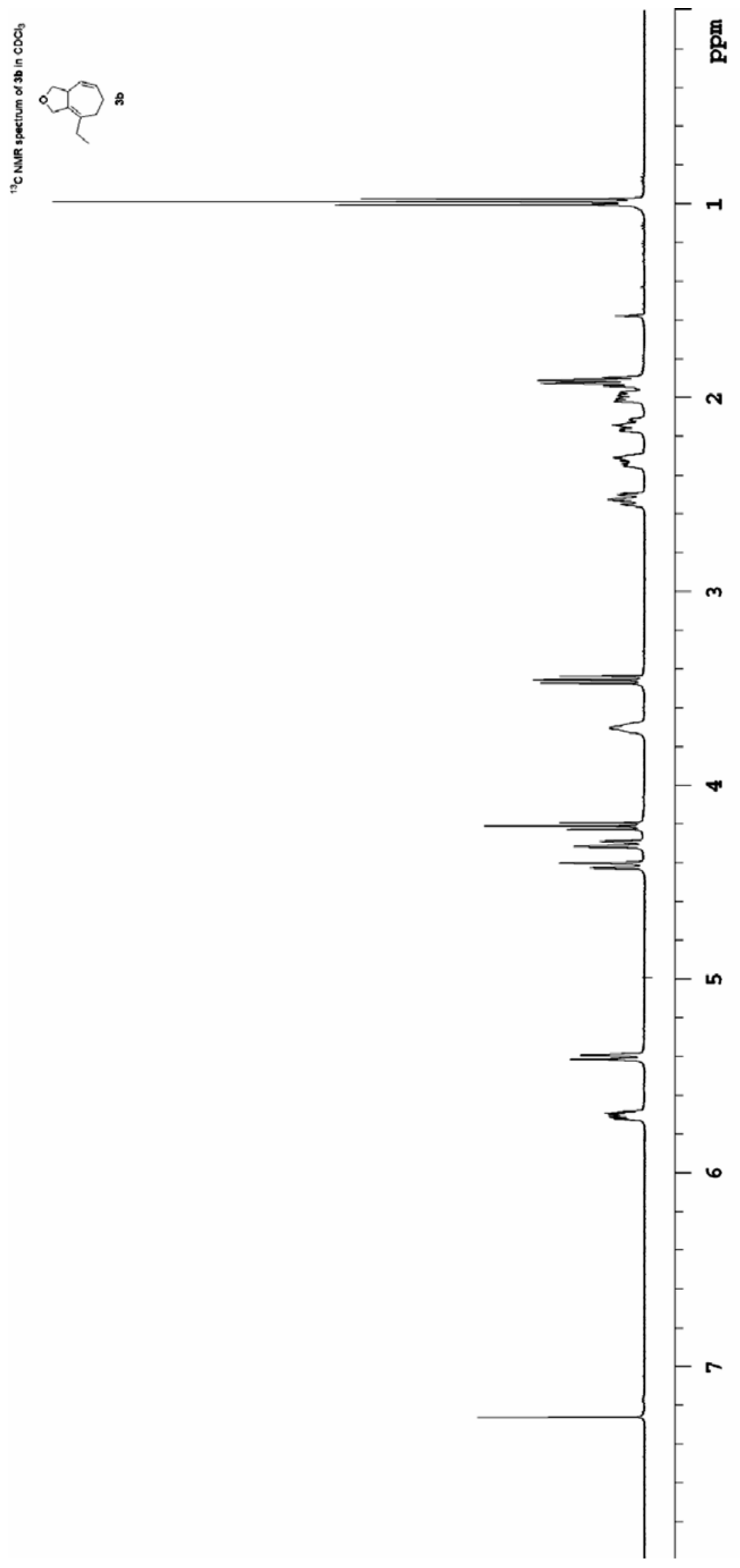




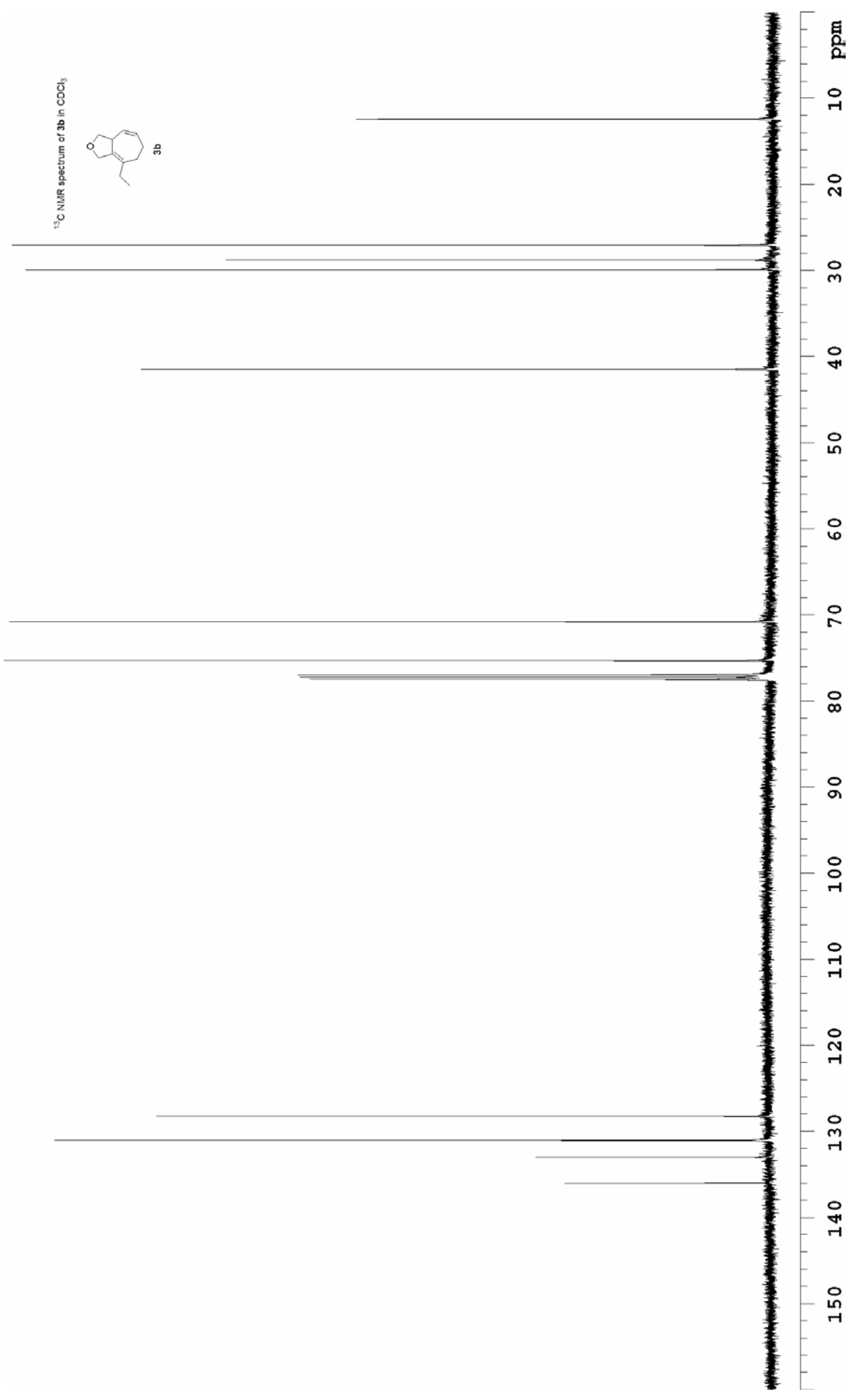



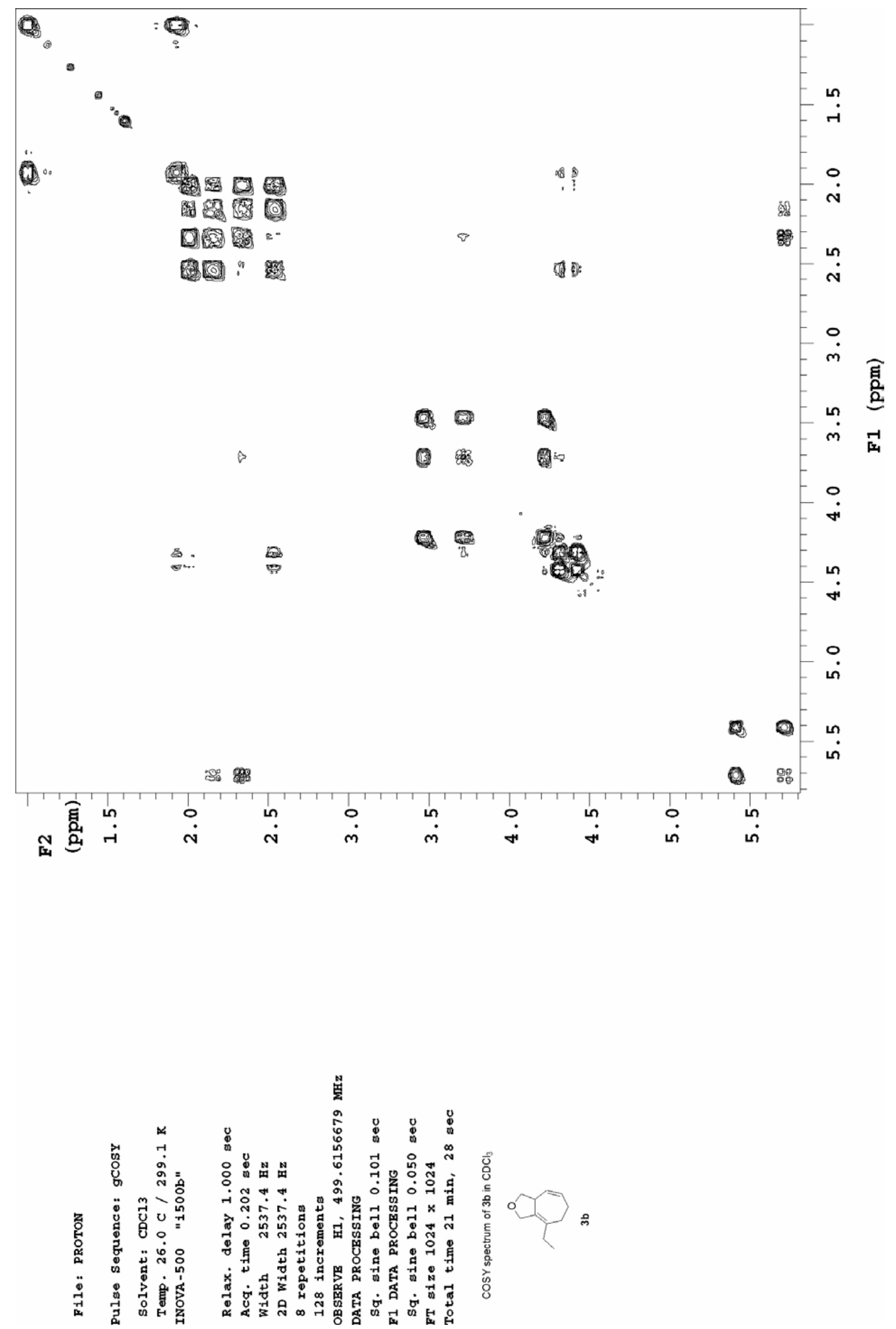

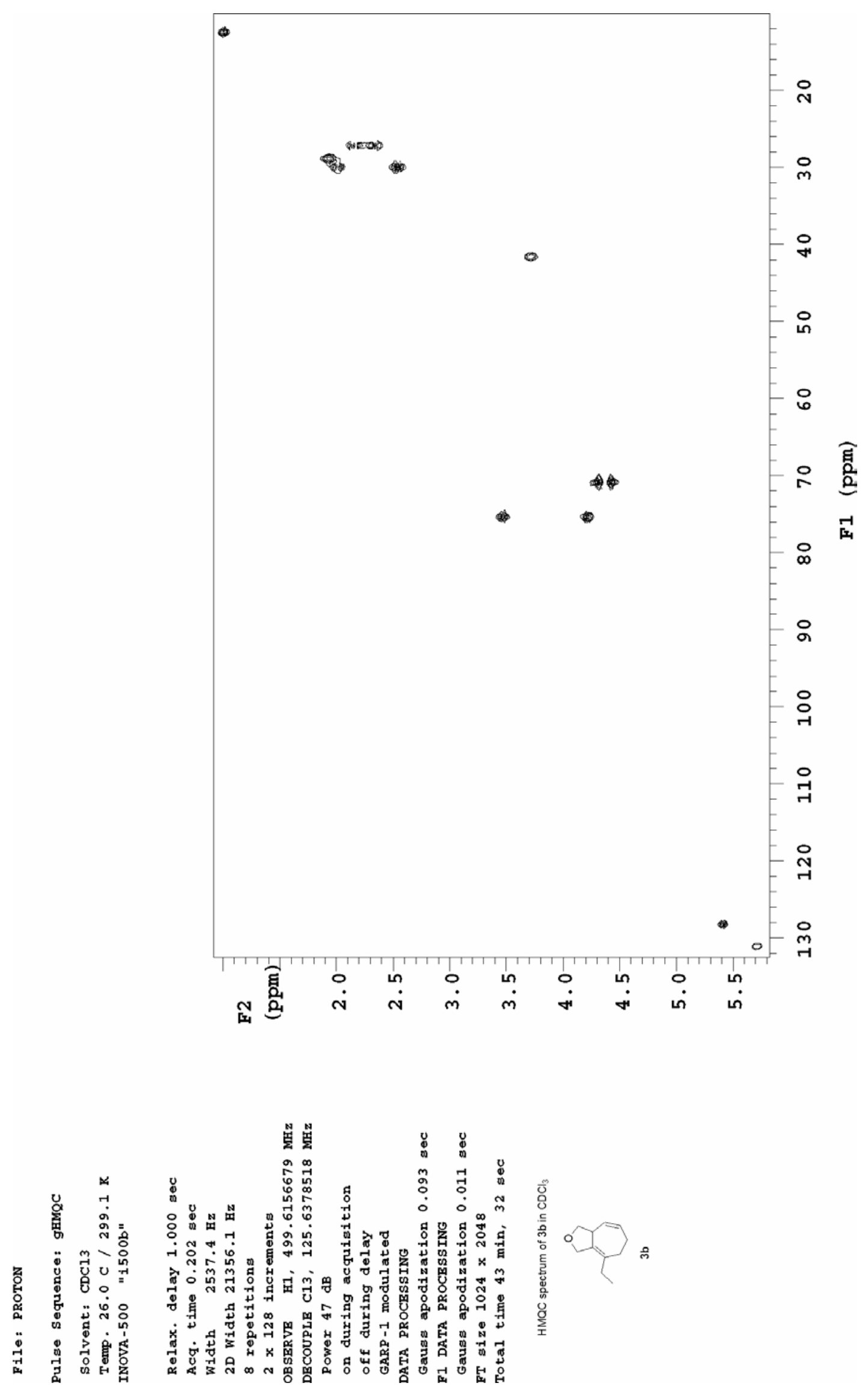

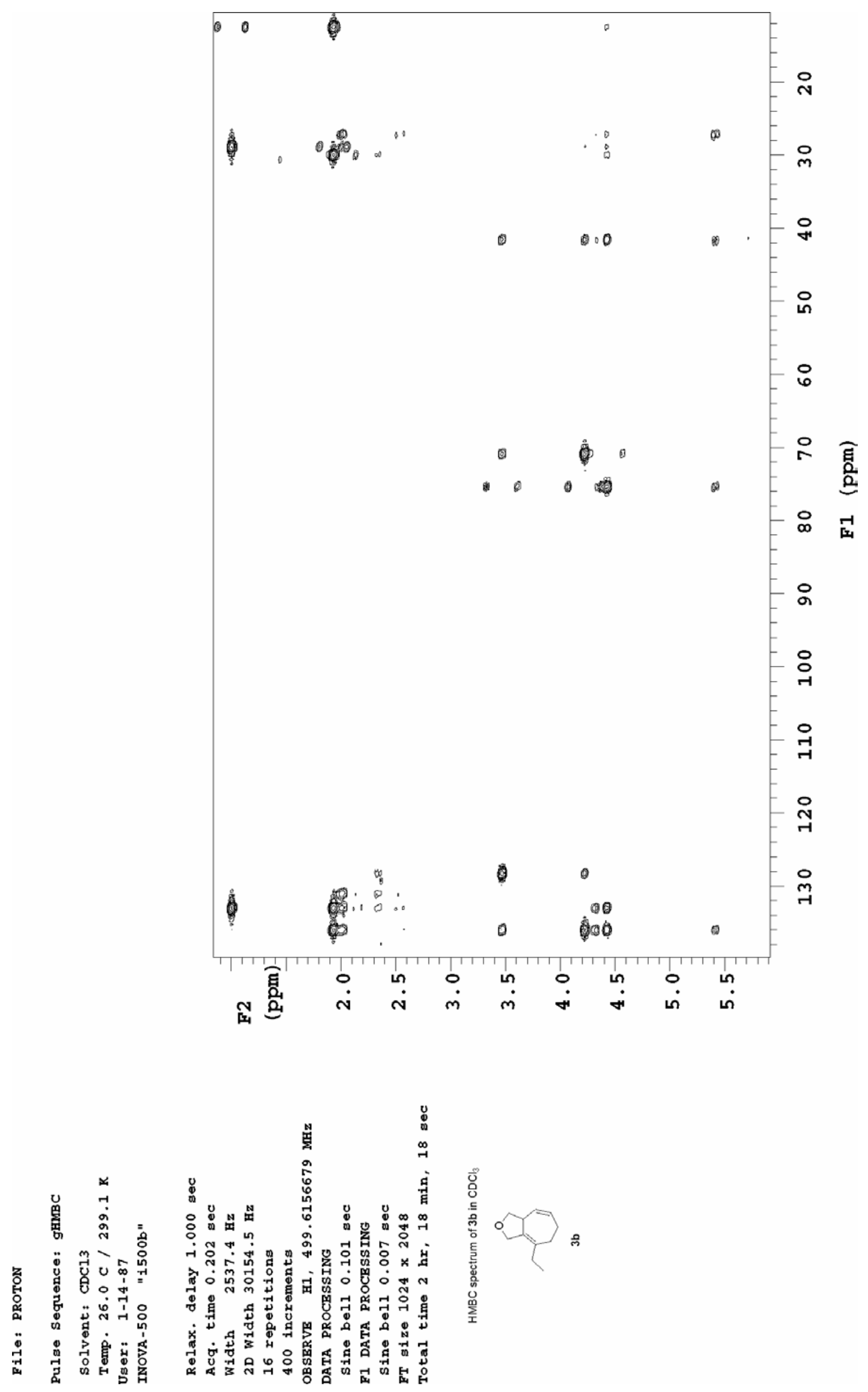


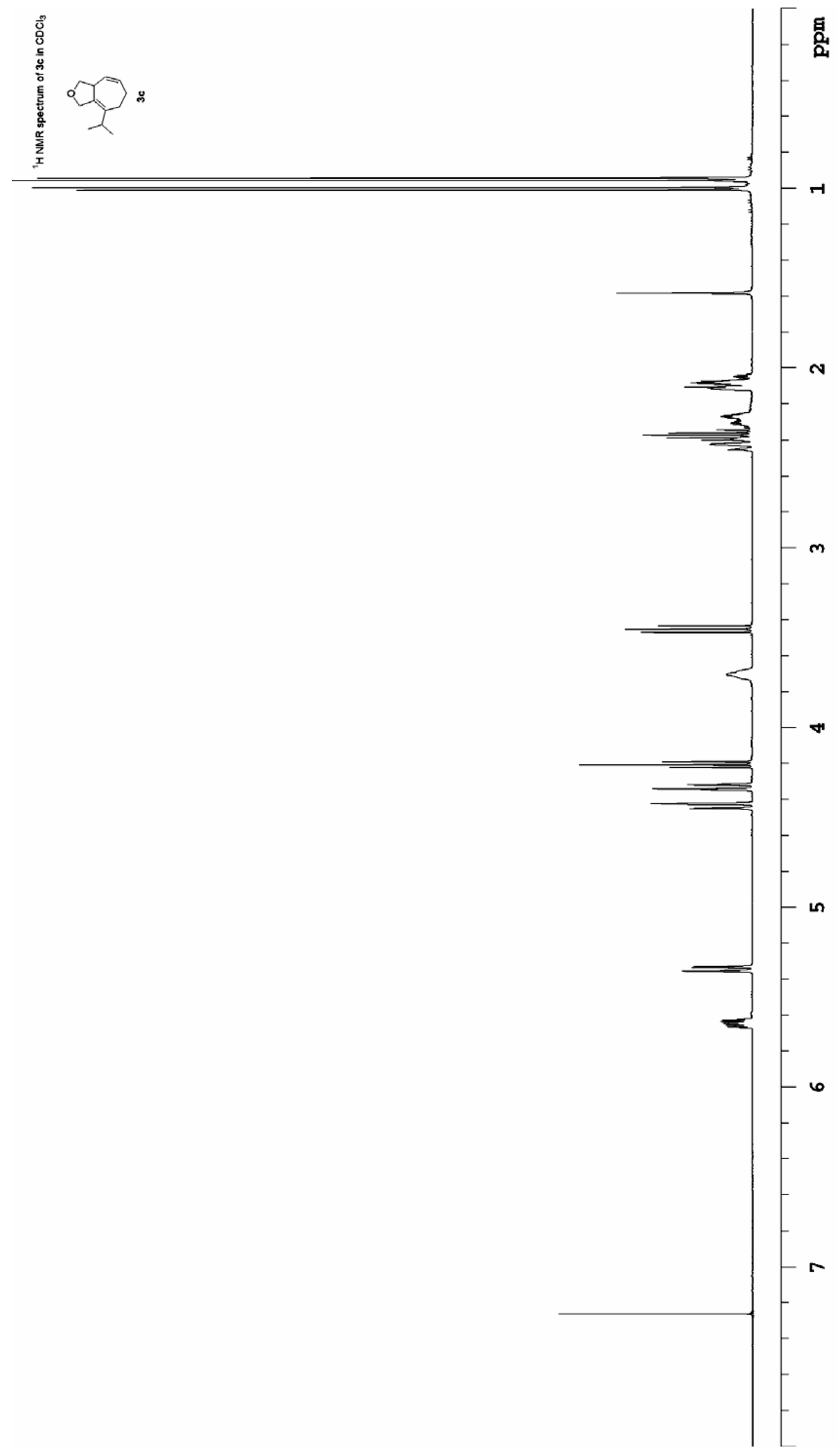




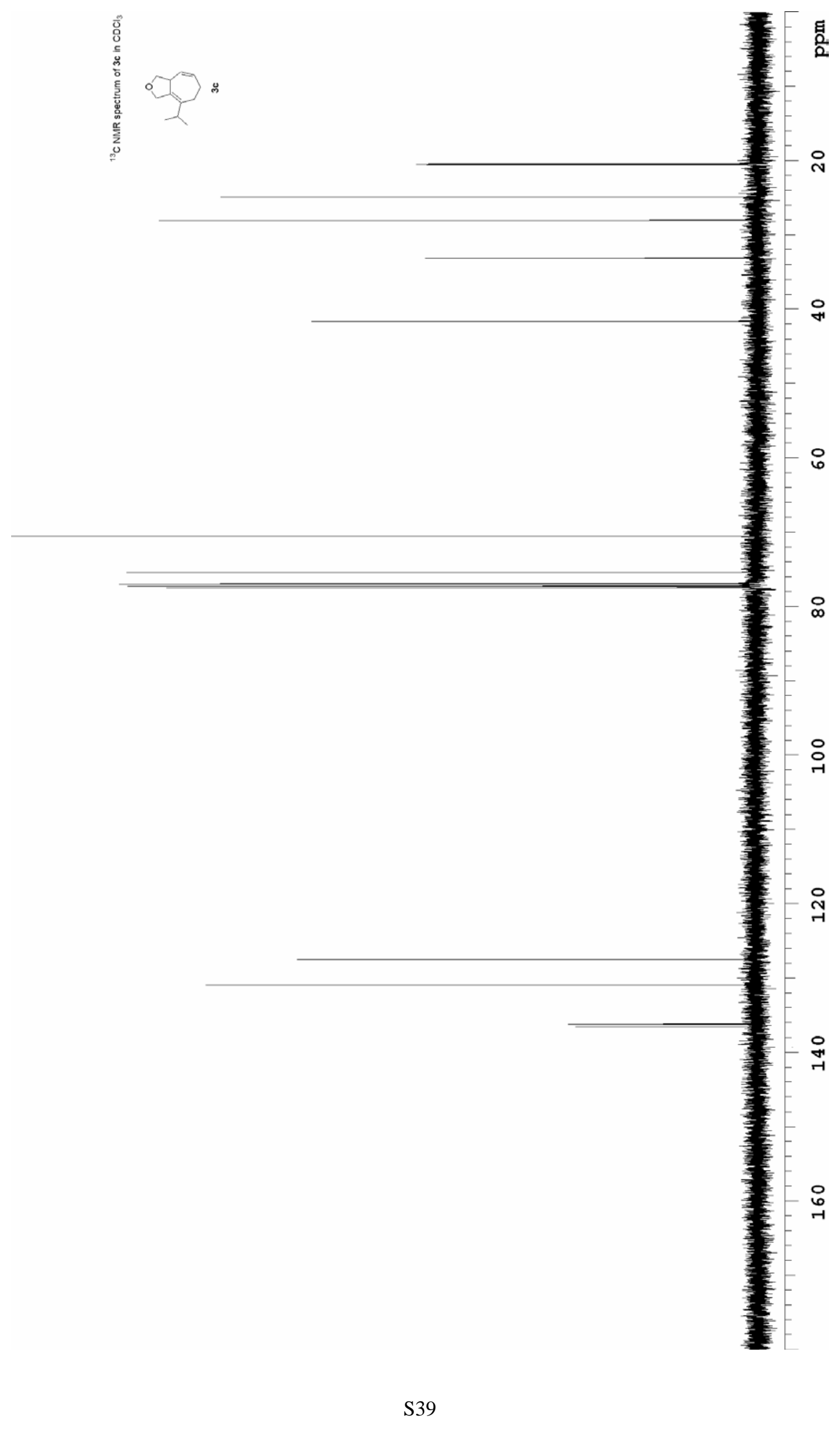



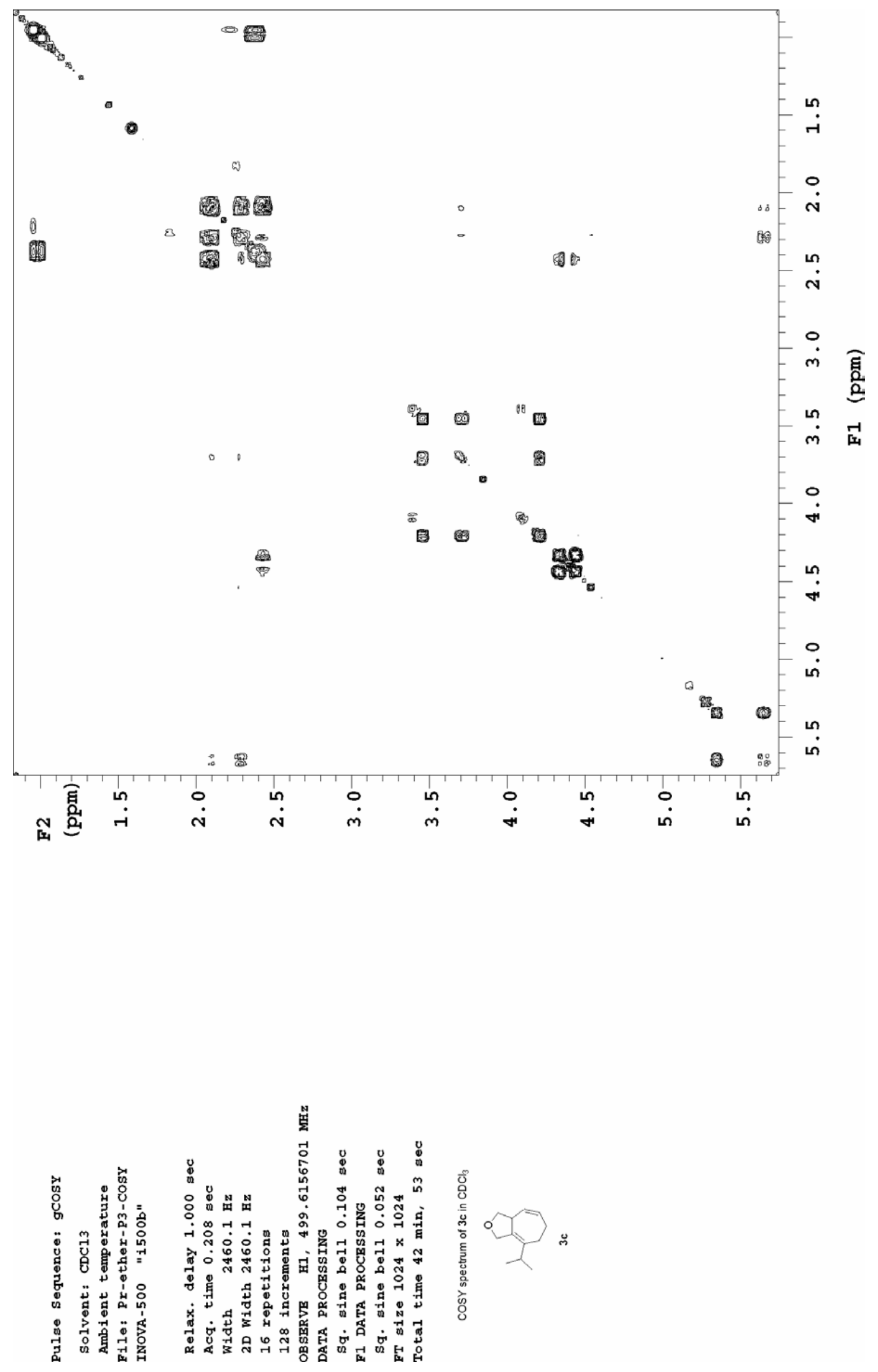

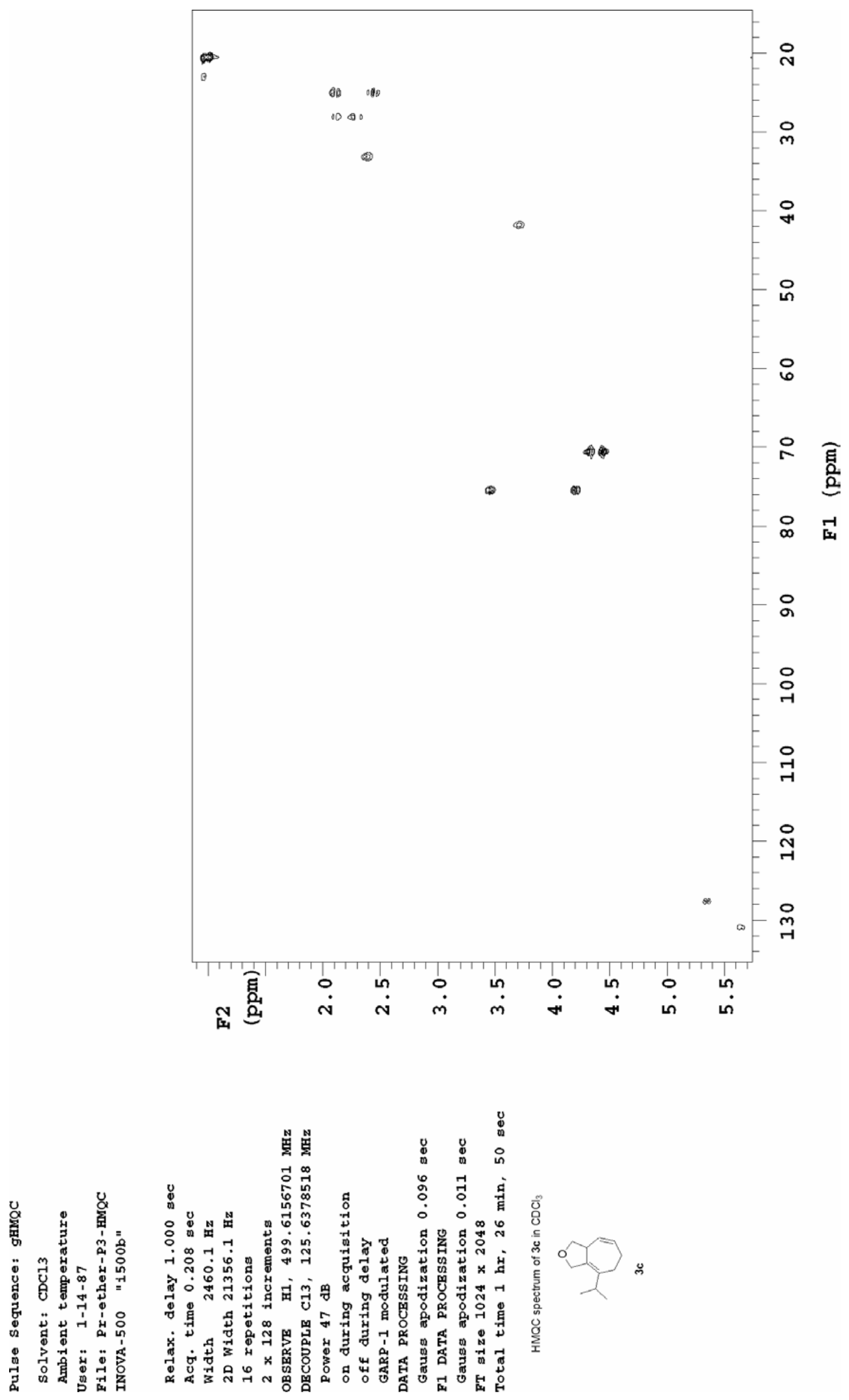

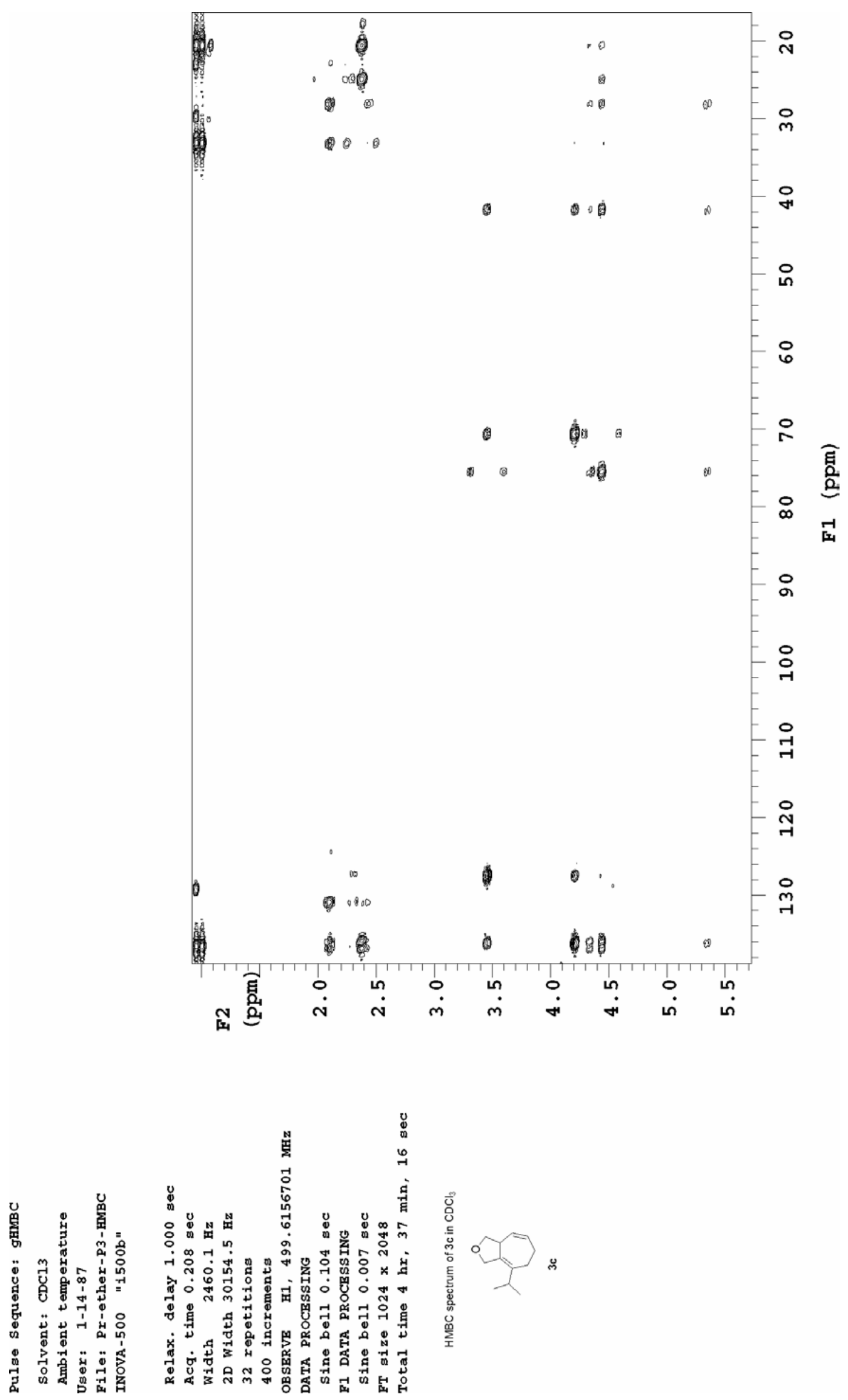


$$
1
$$



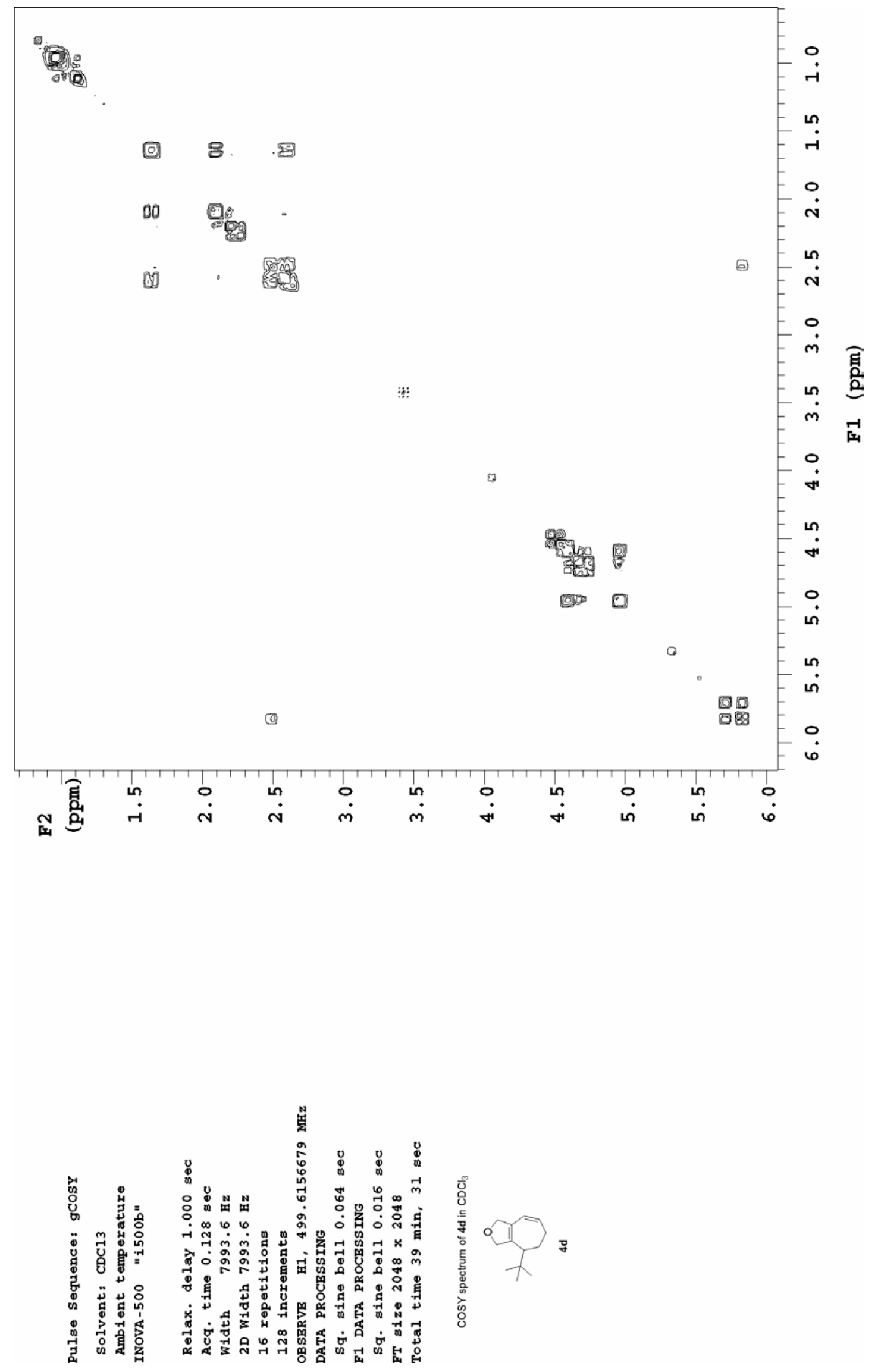

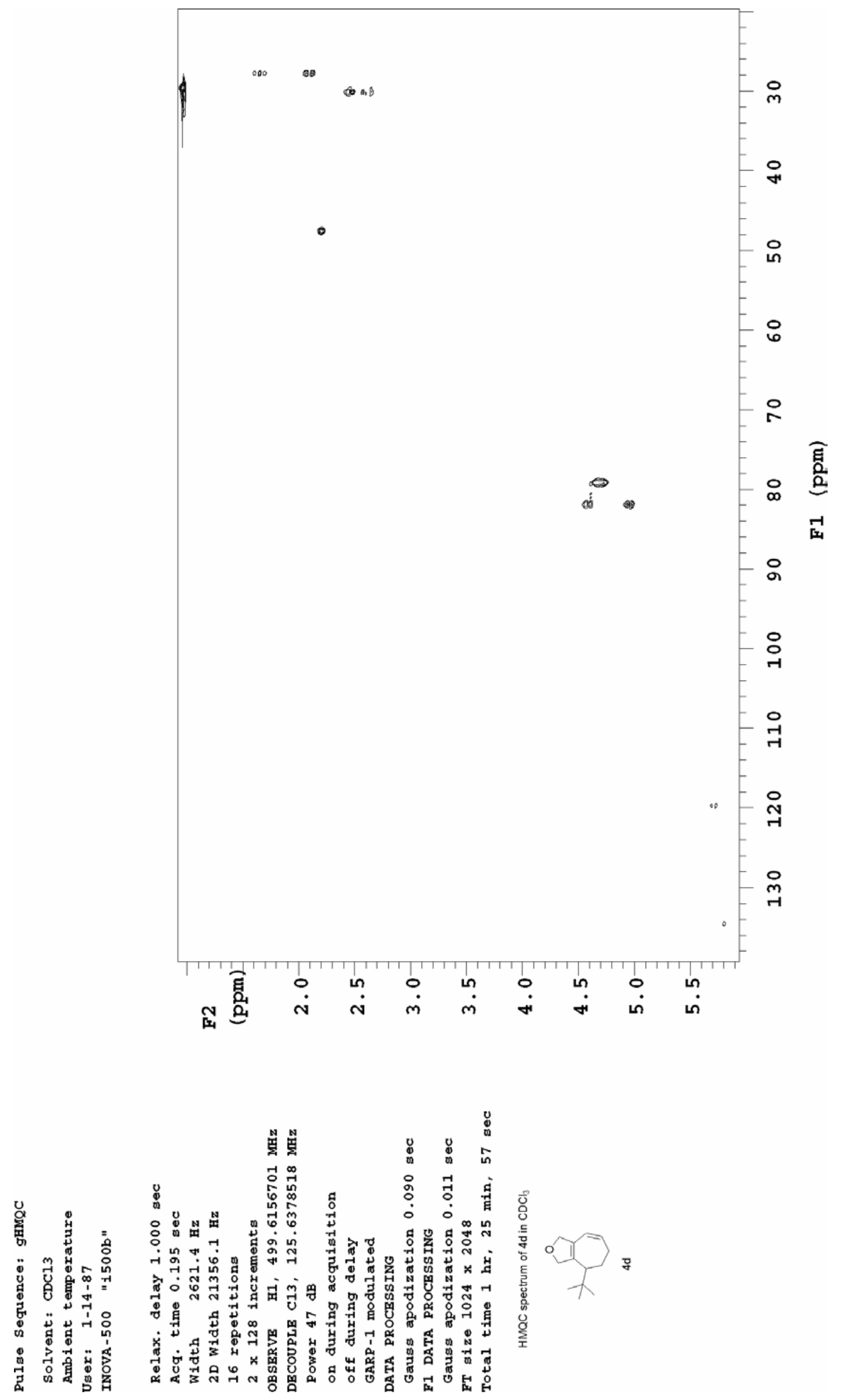

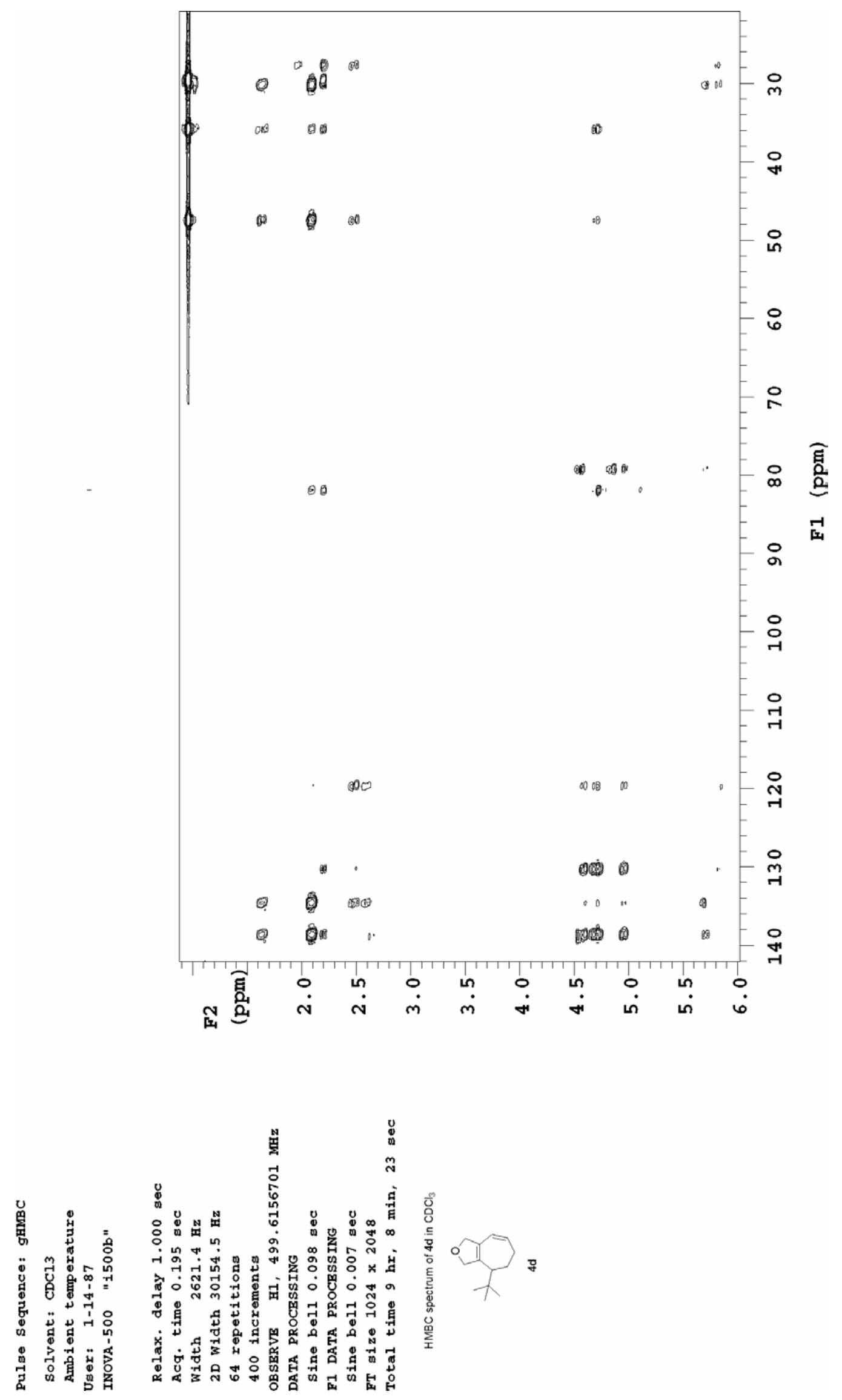


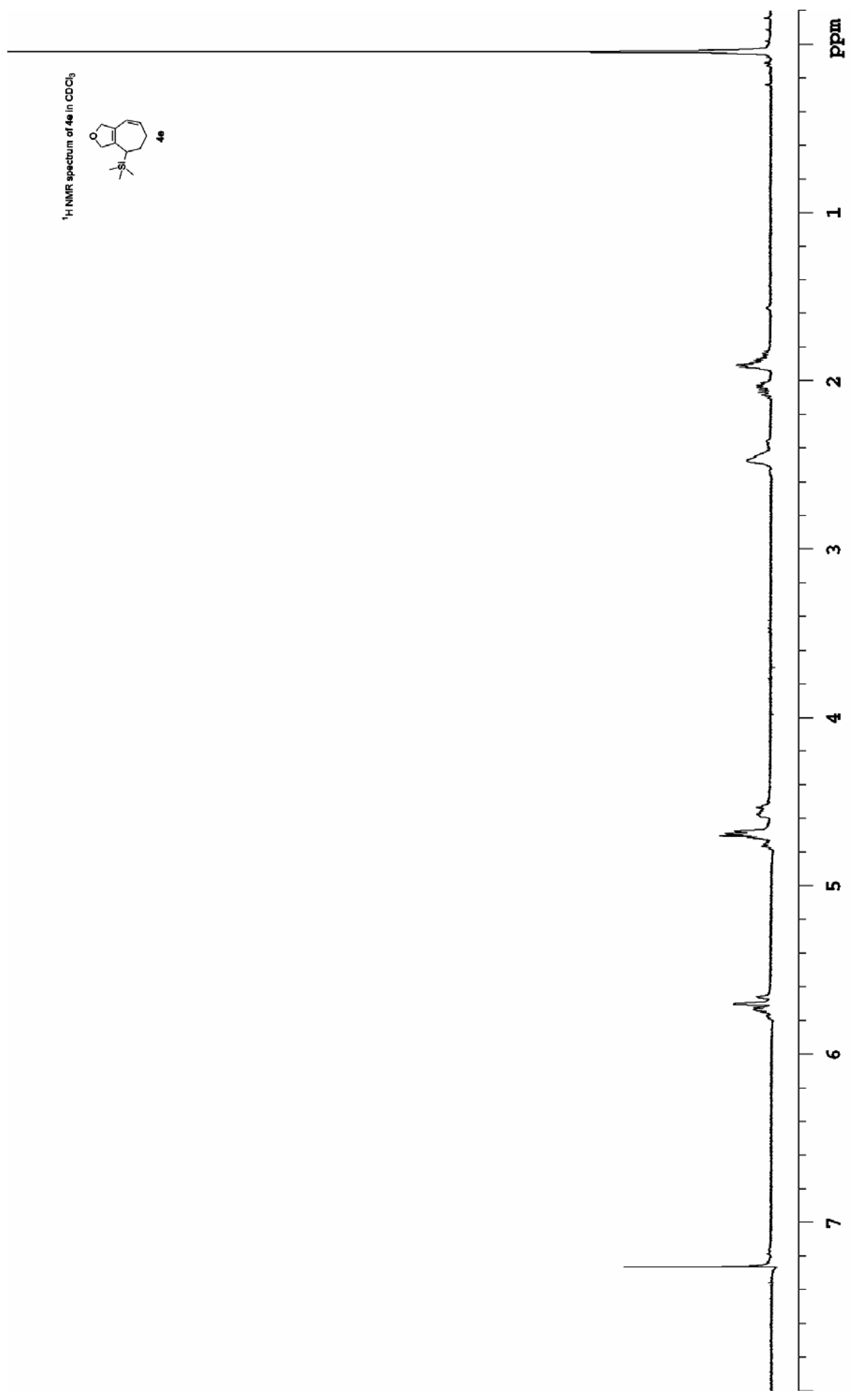



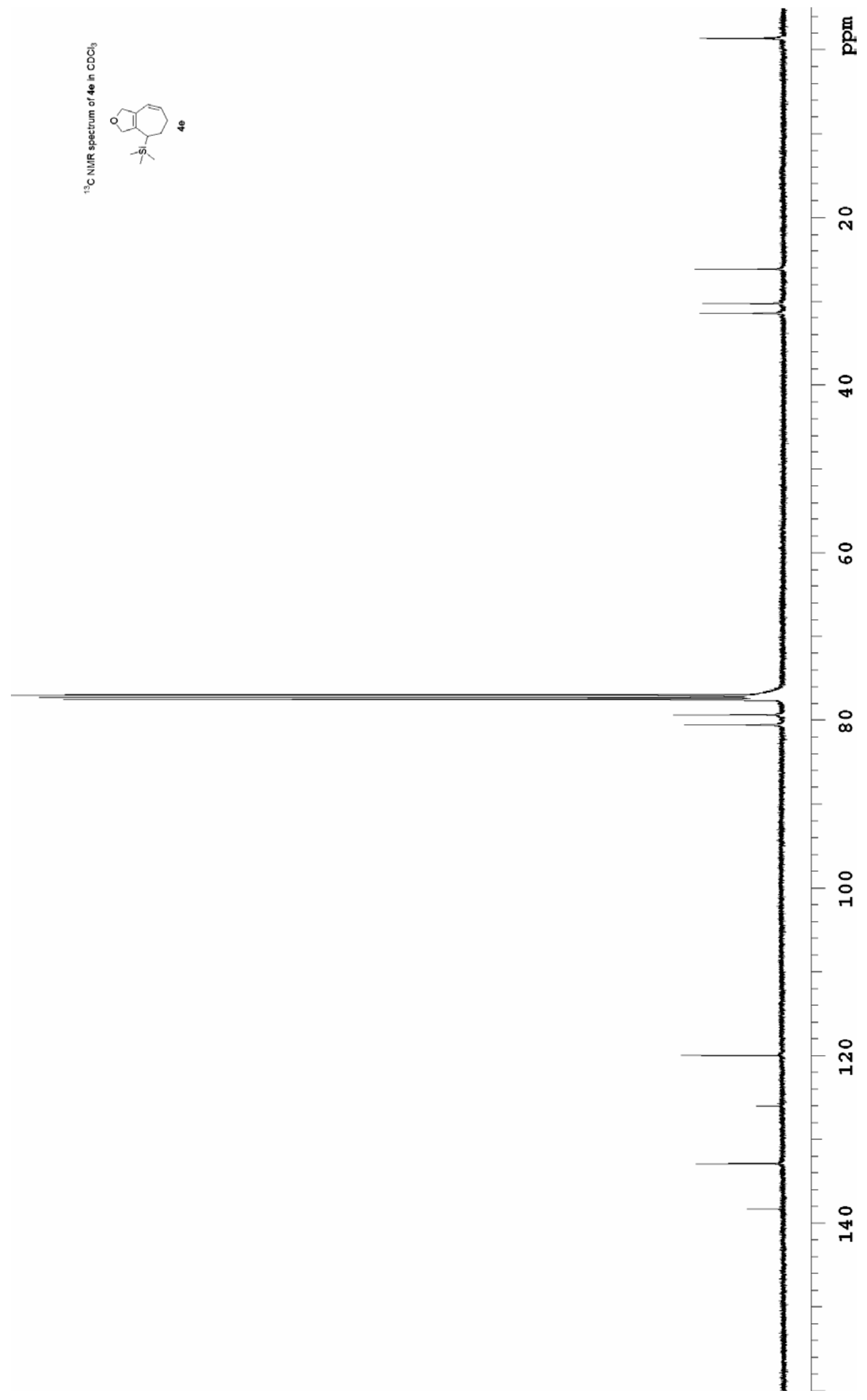

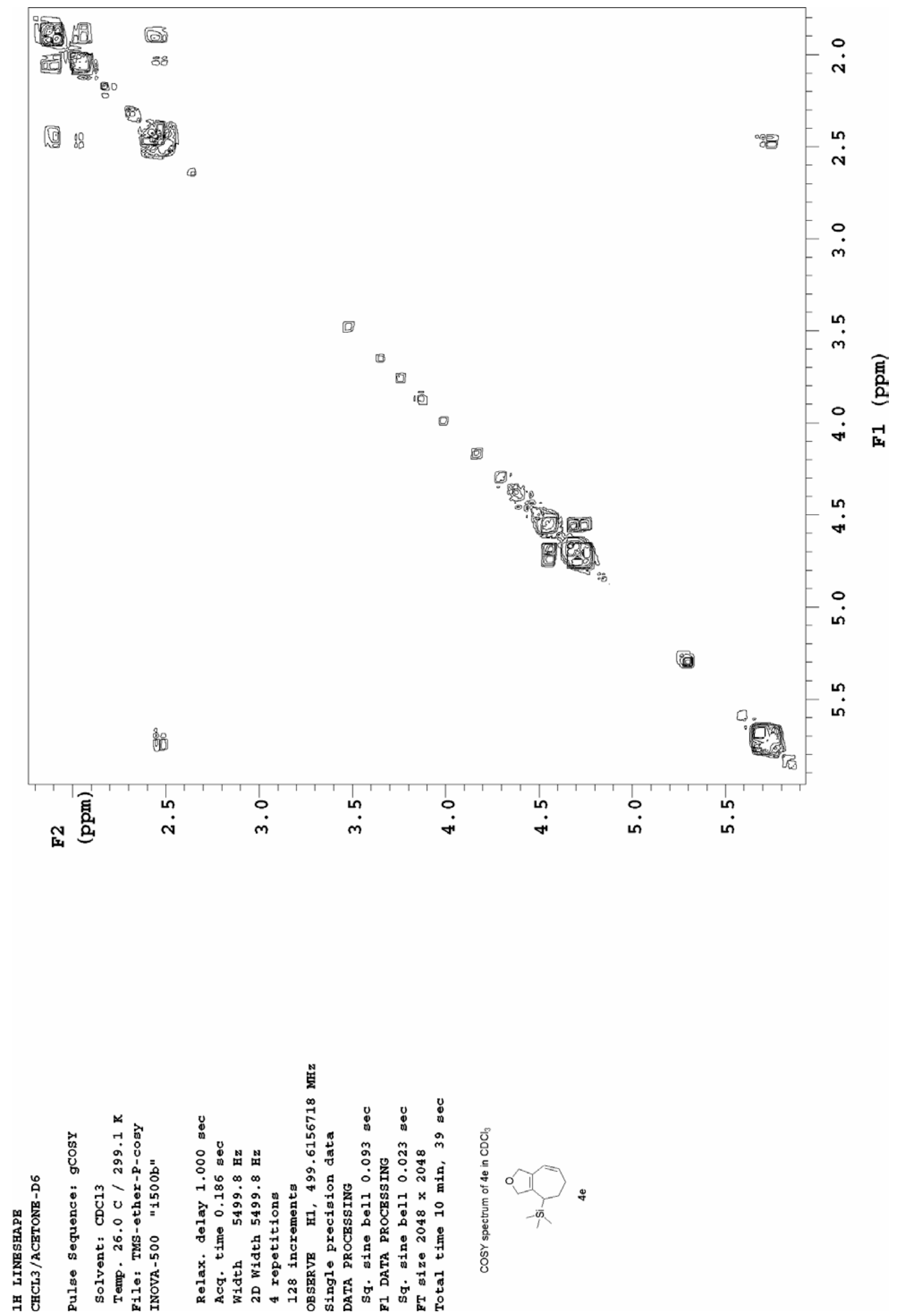

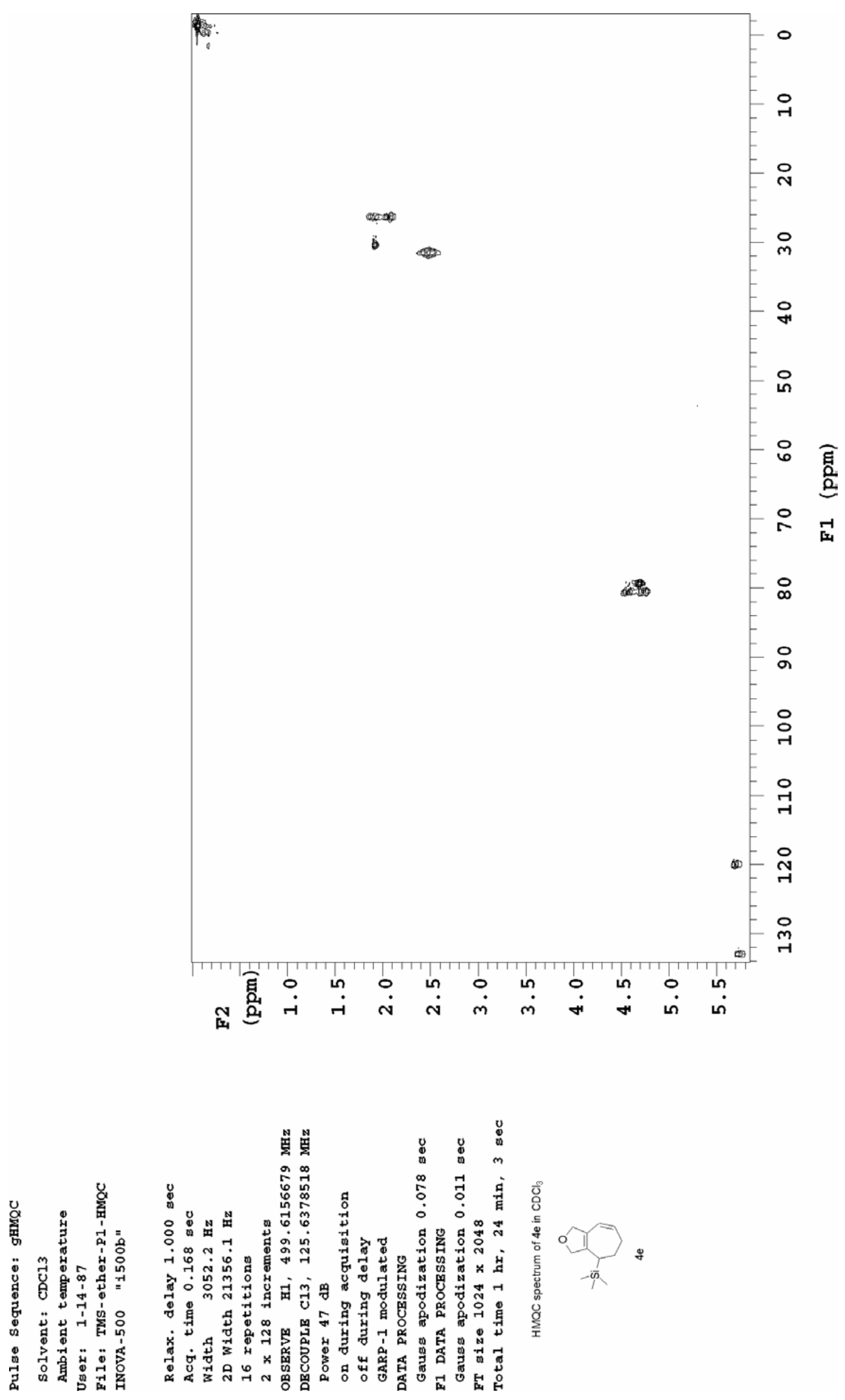

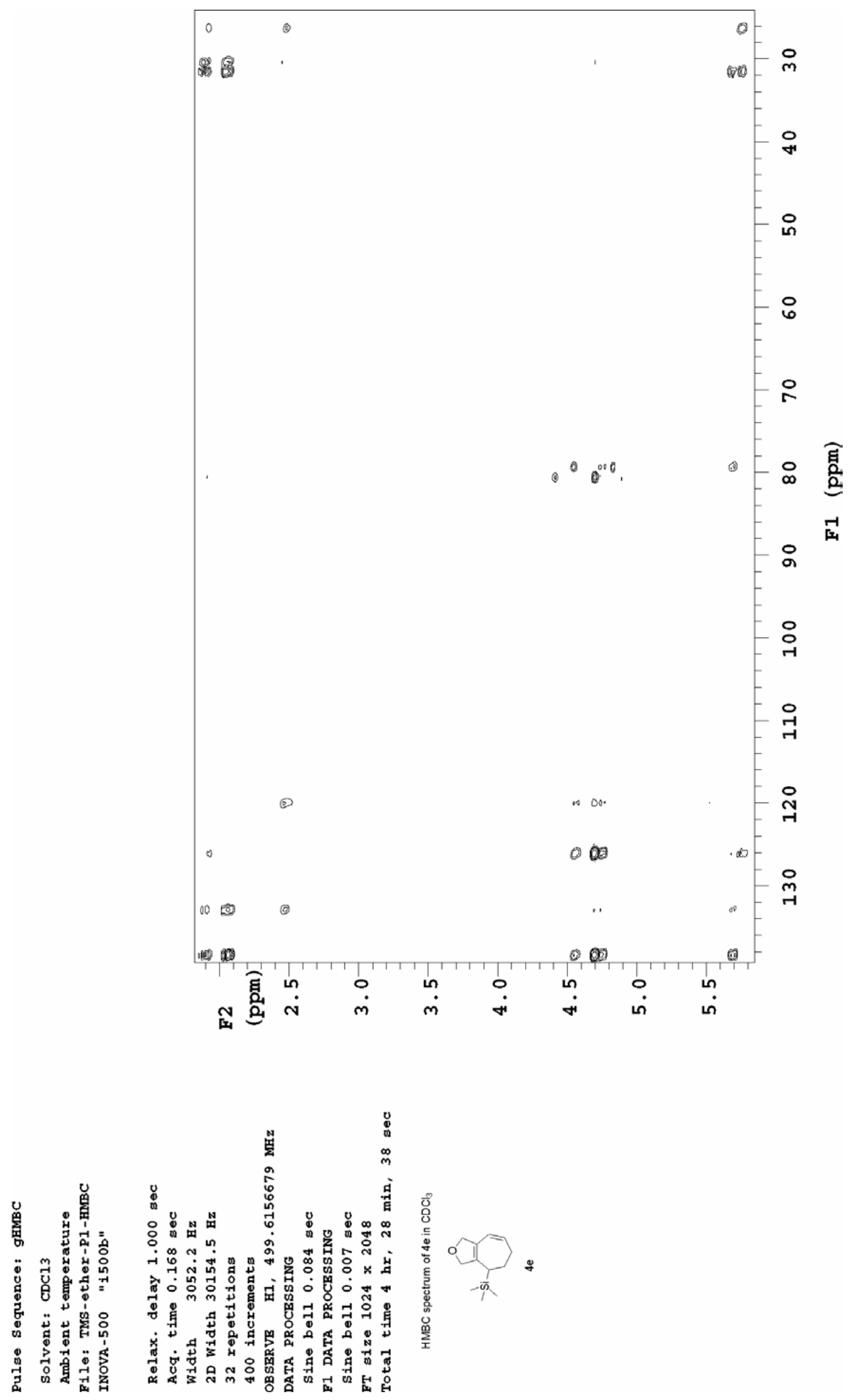

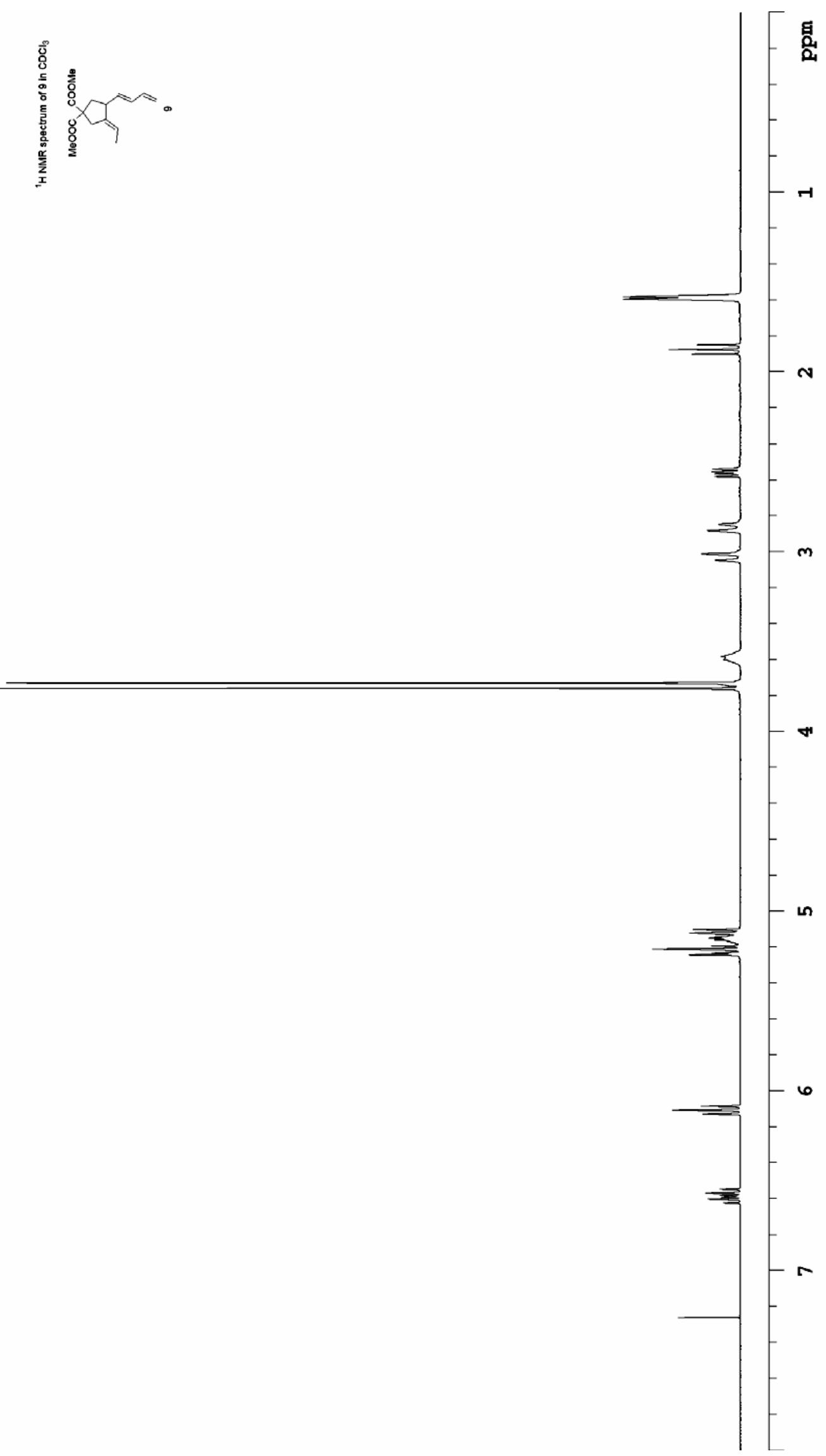


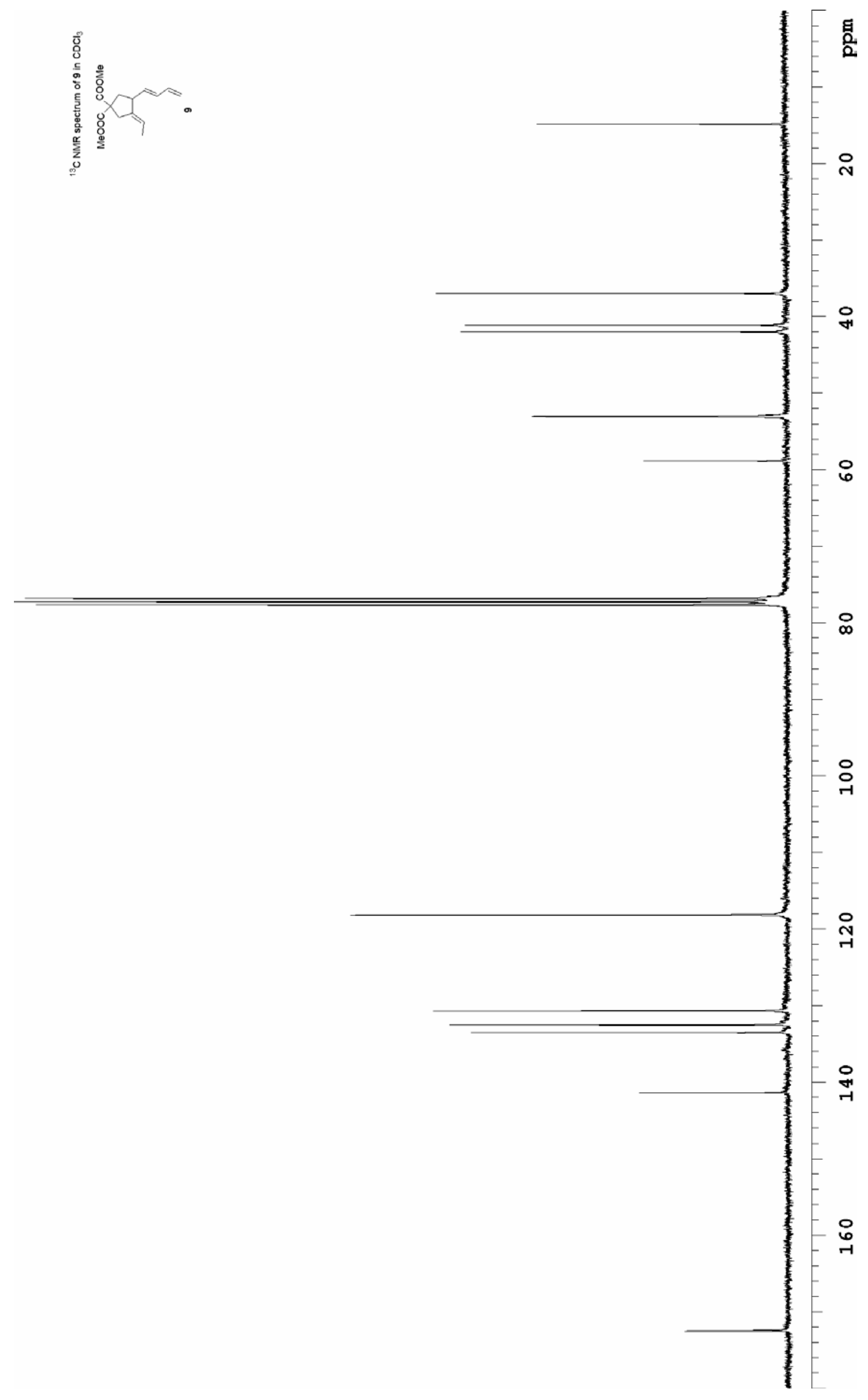



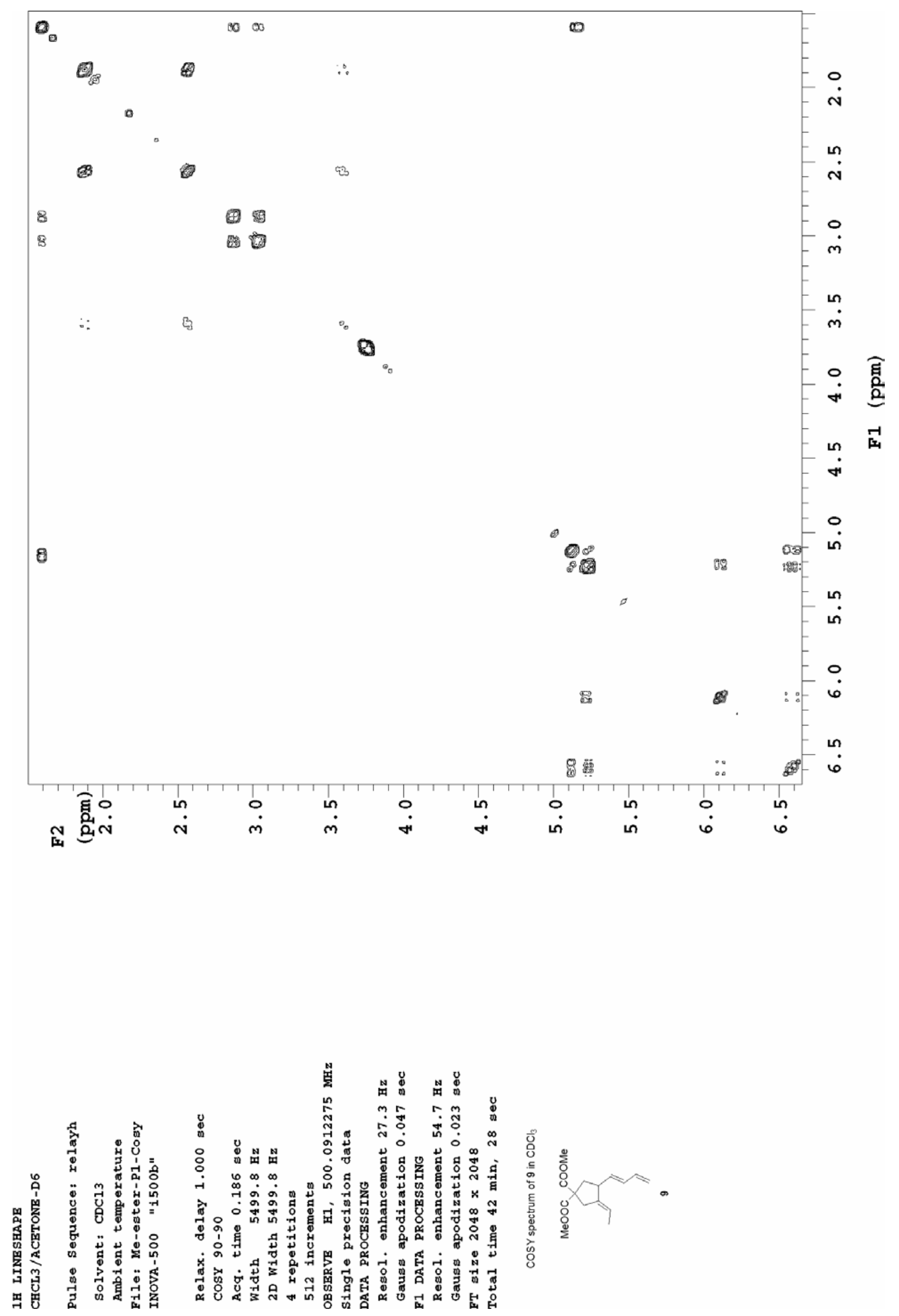

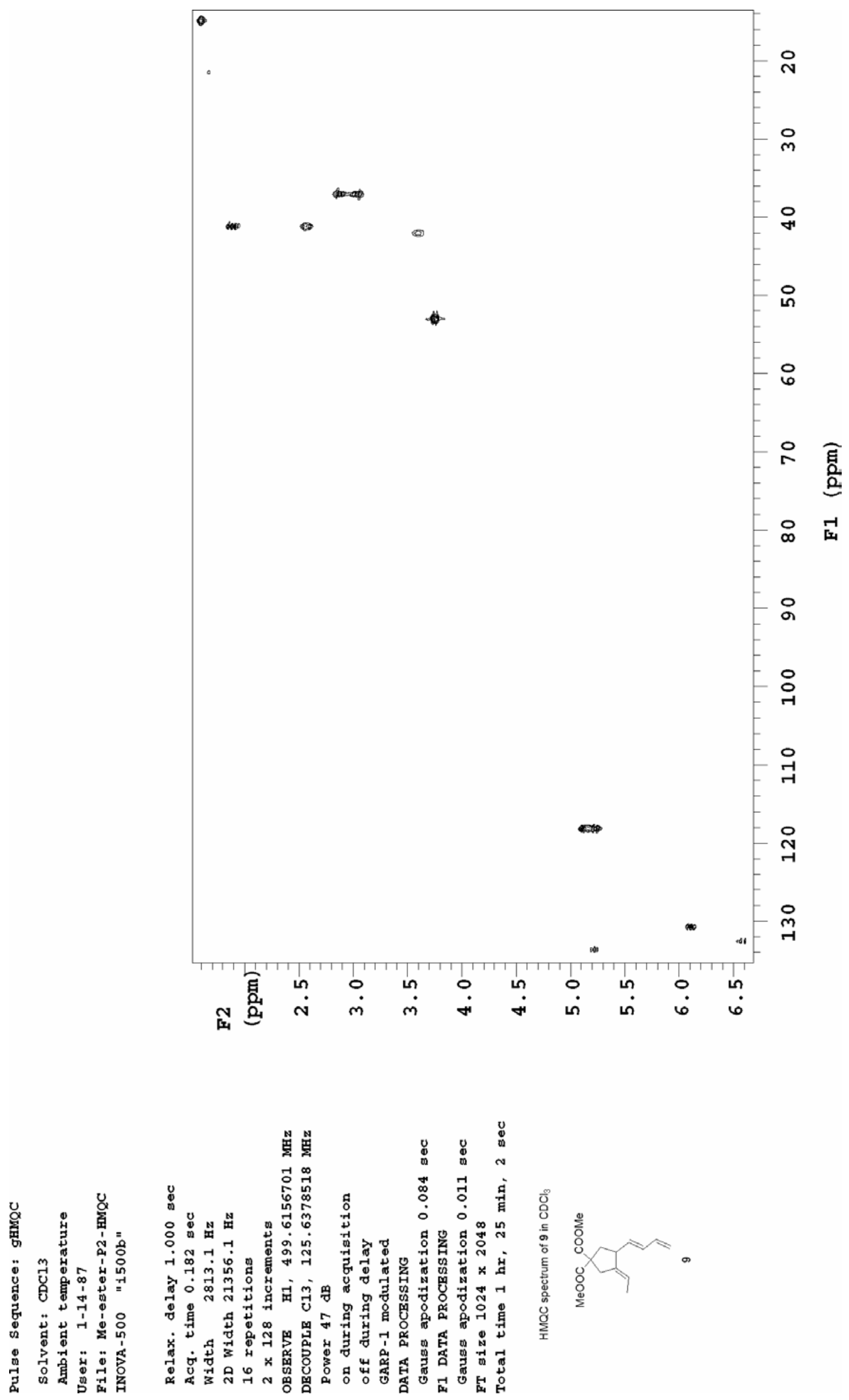

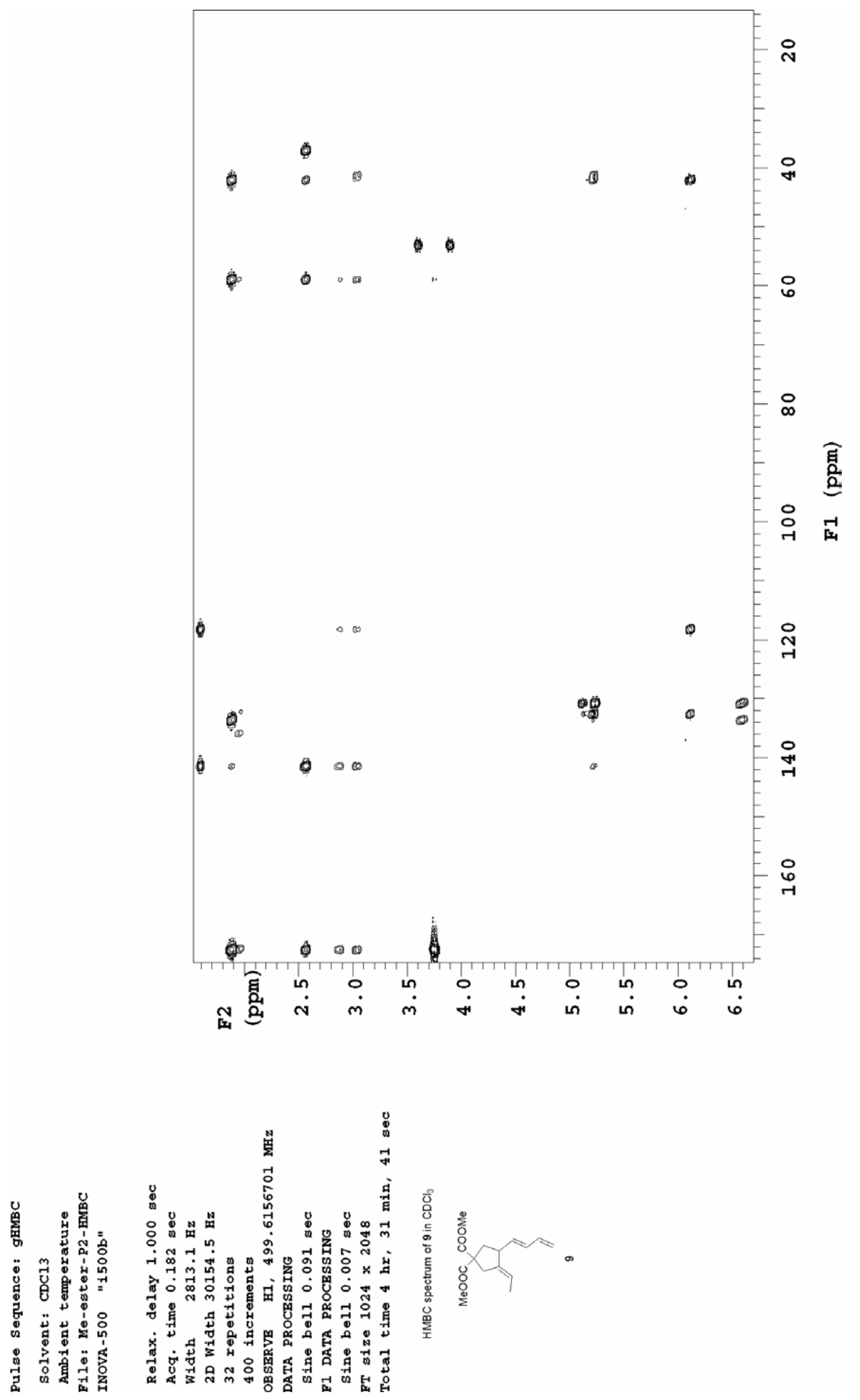


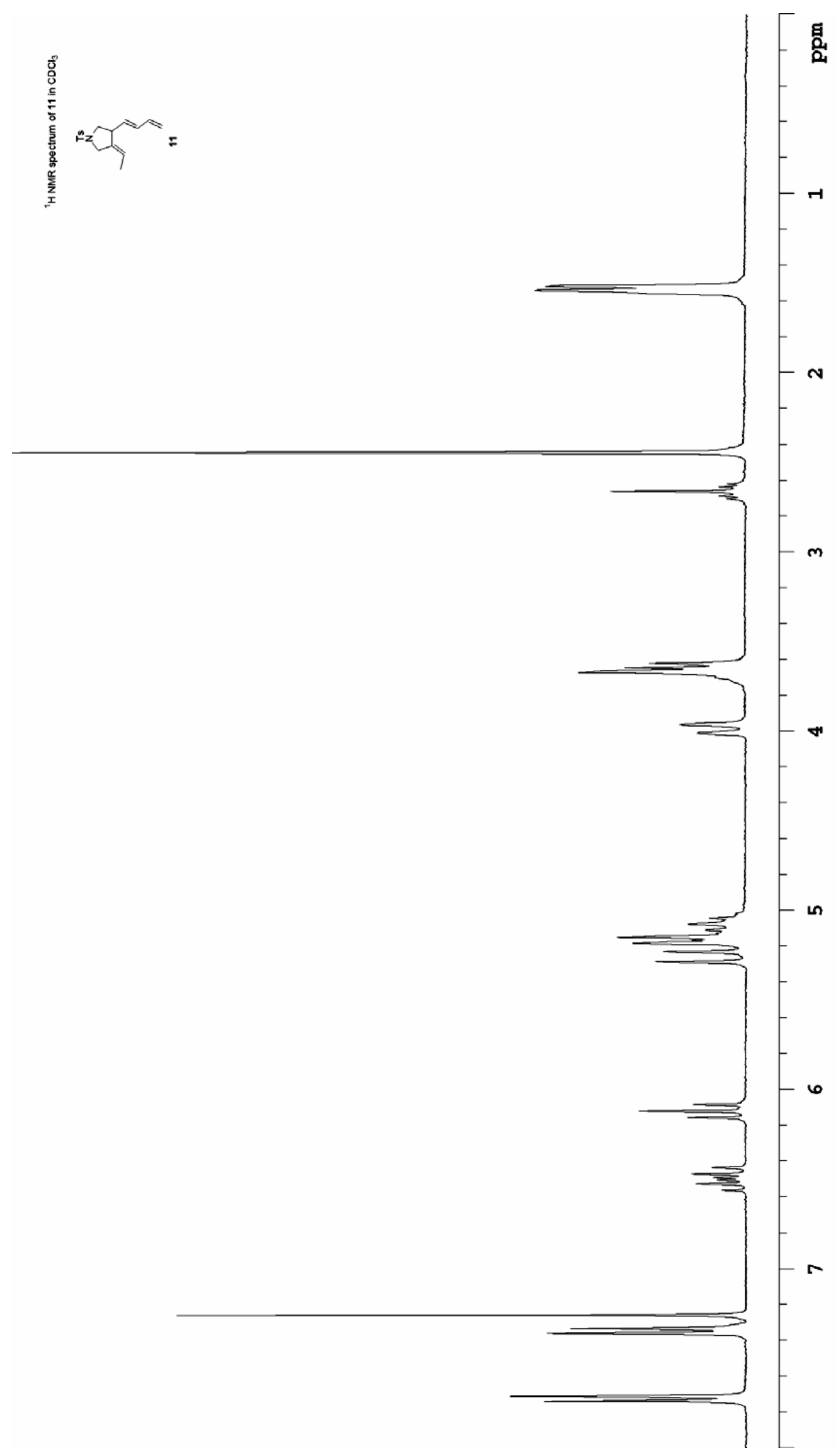



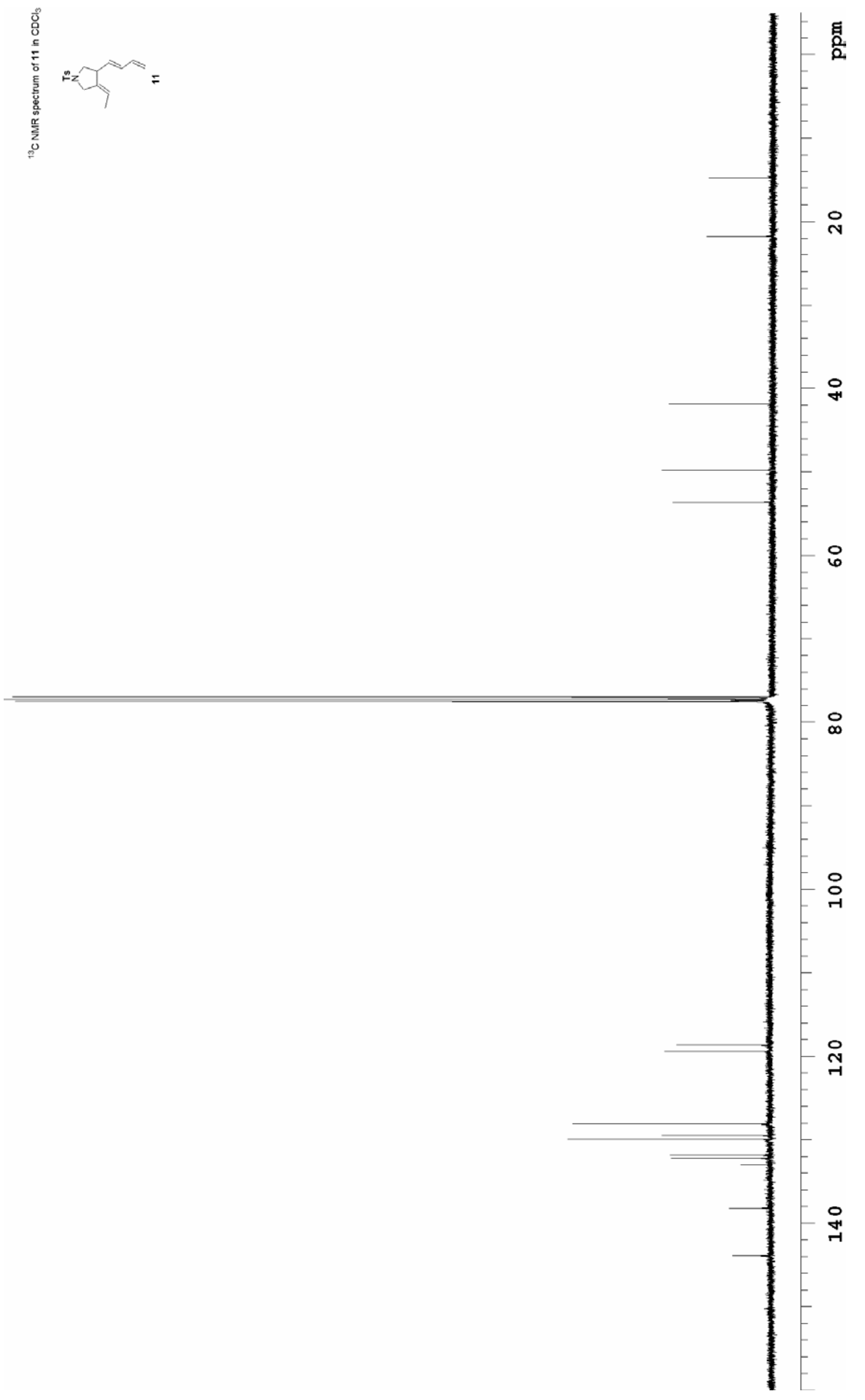

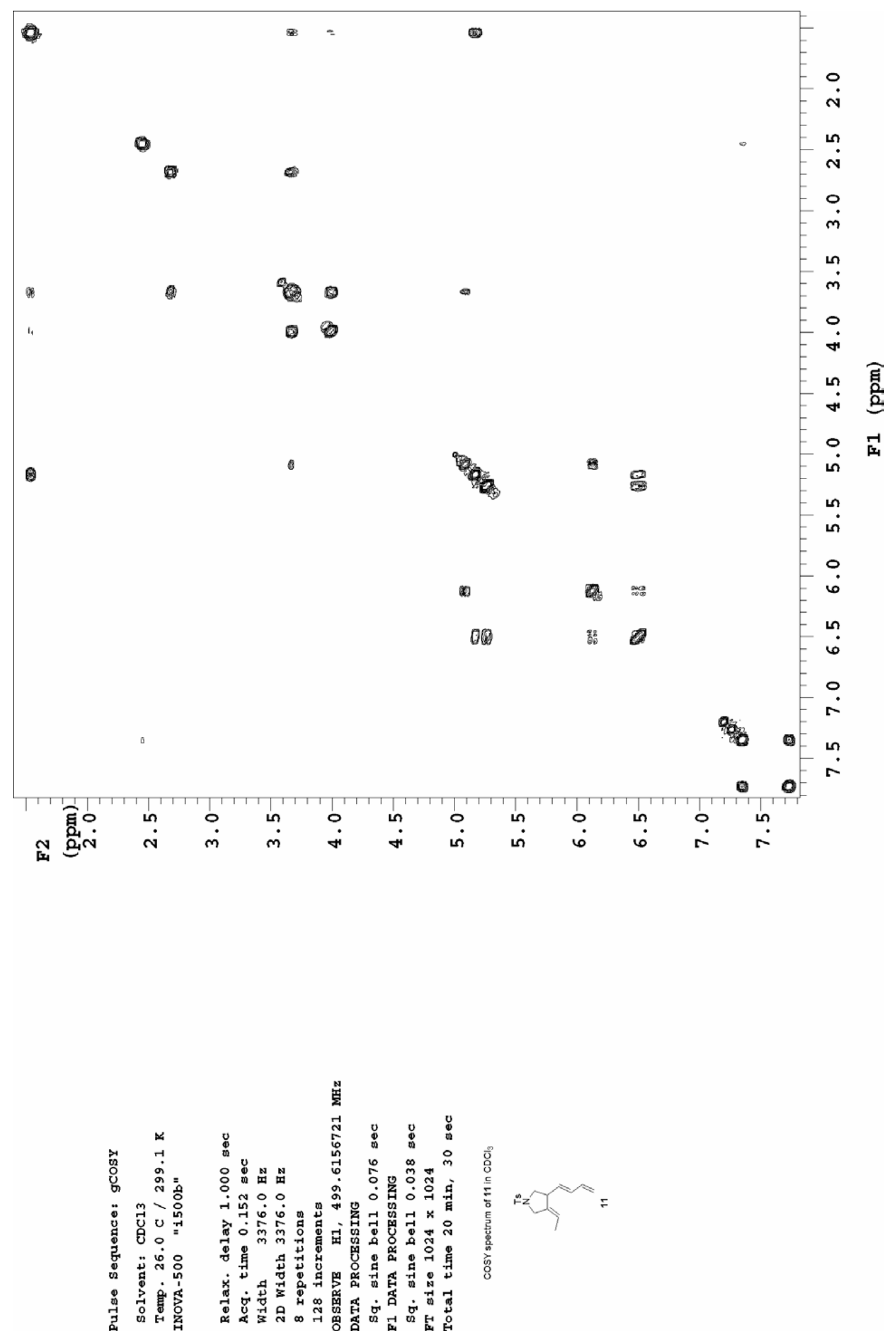

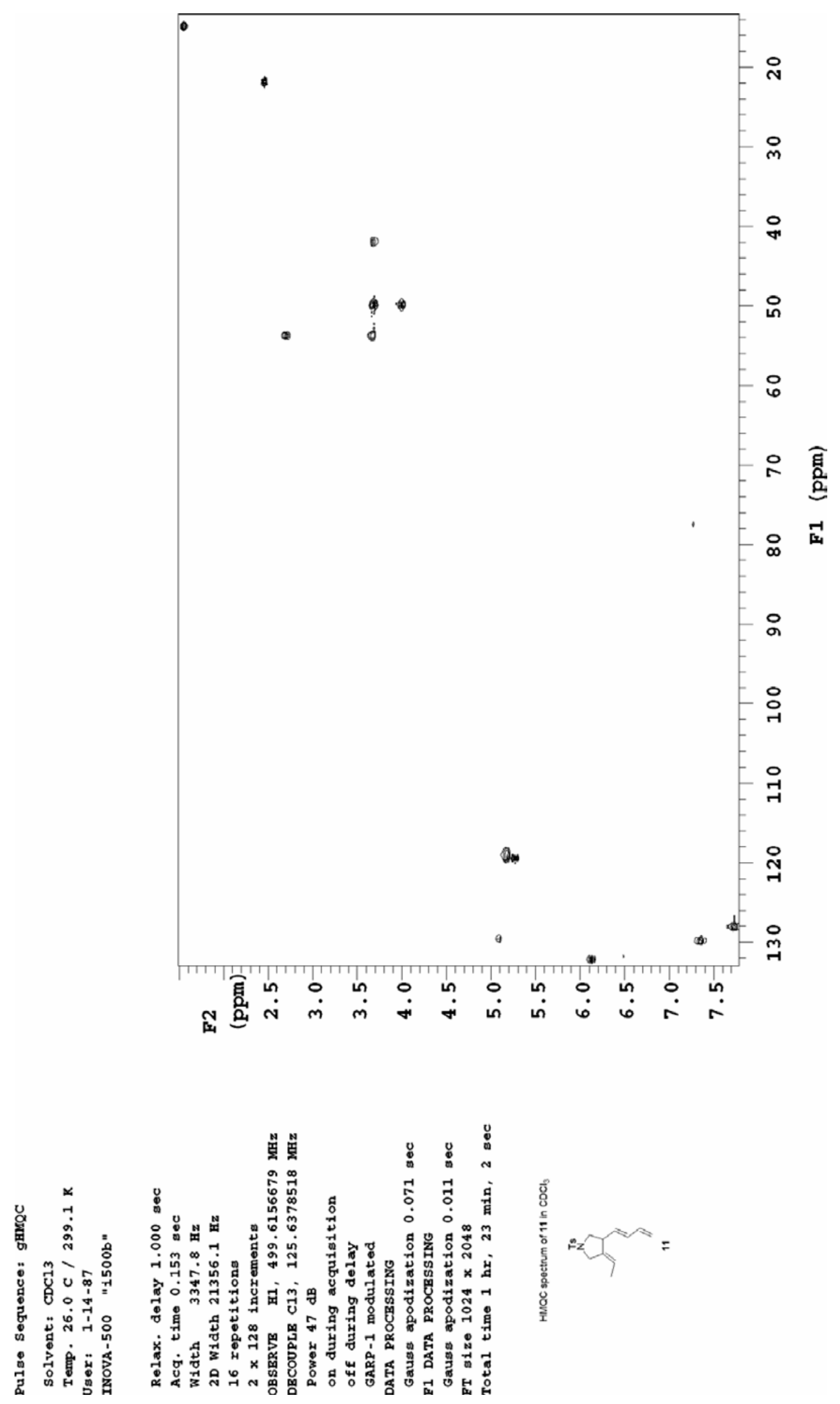

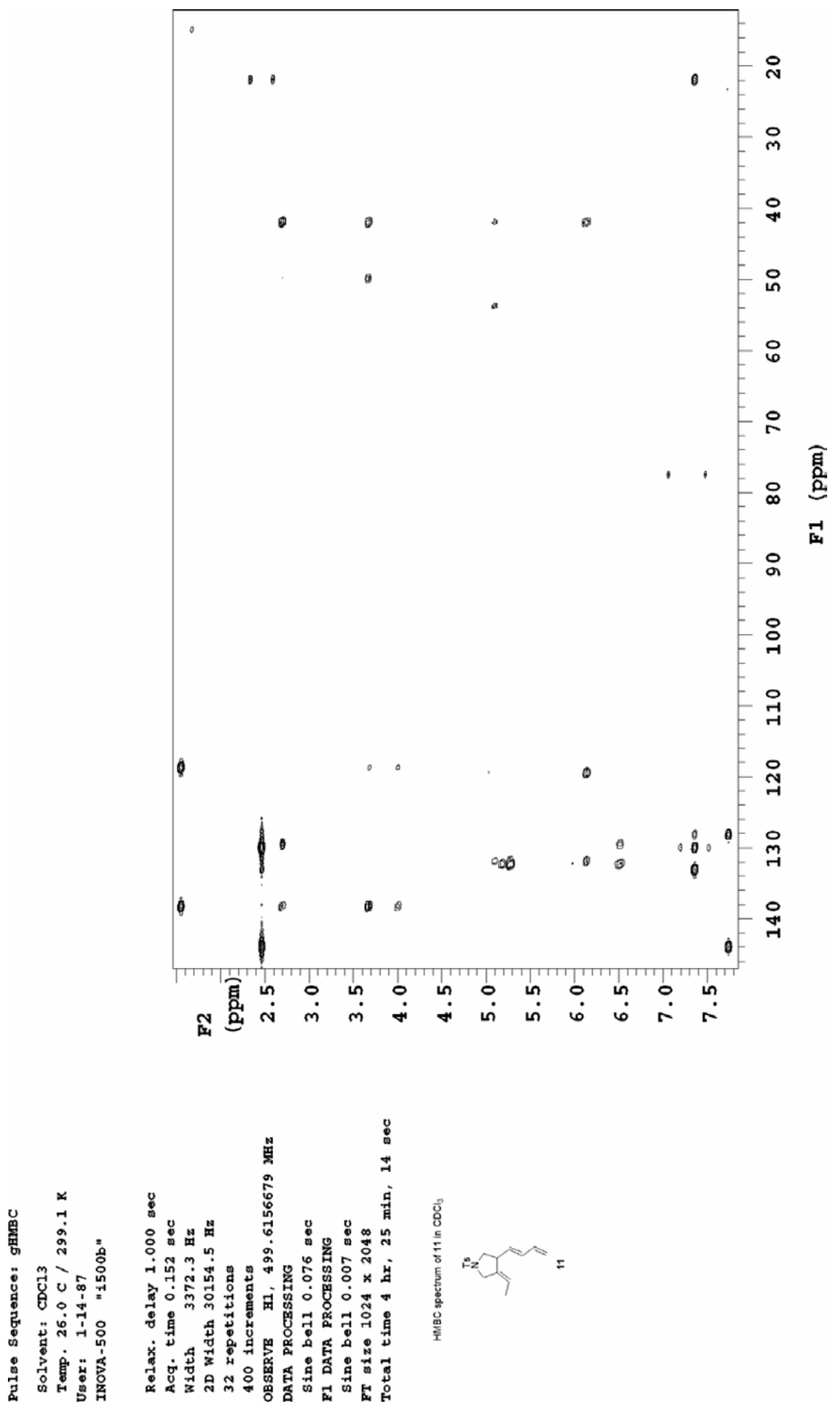

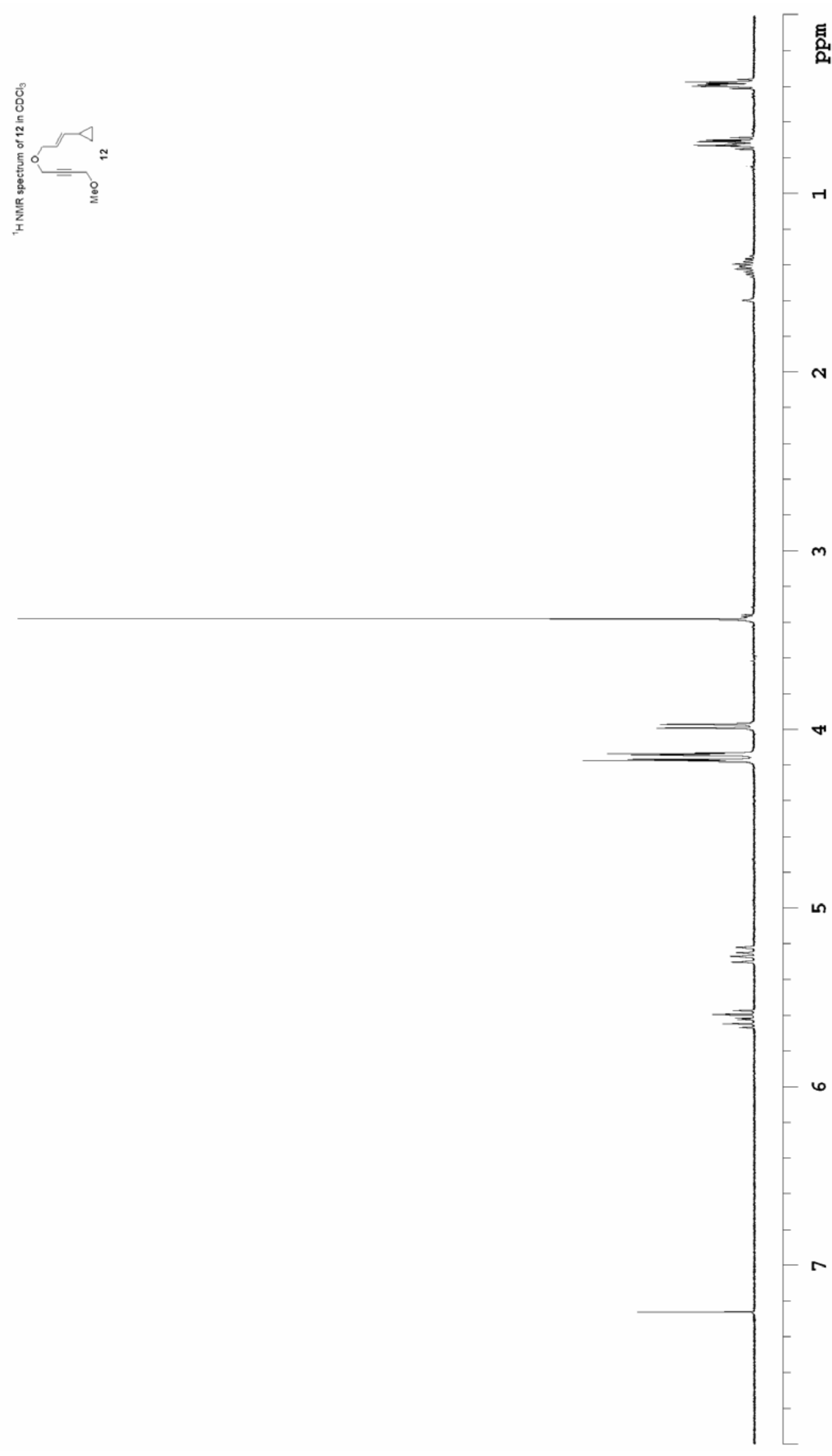

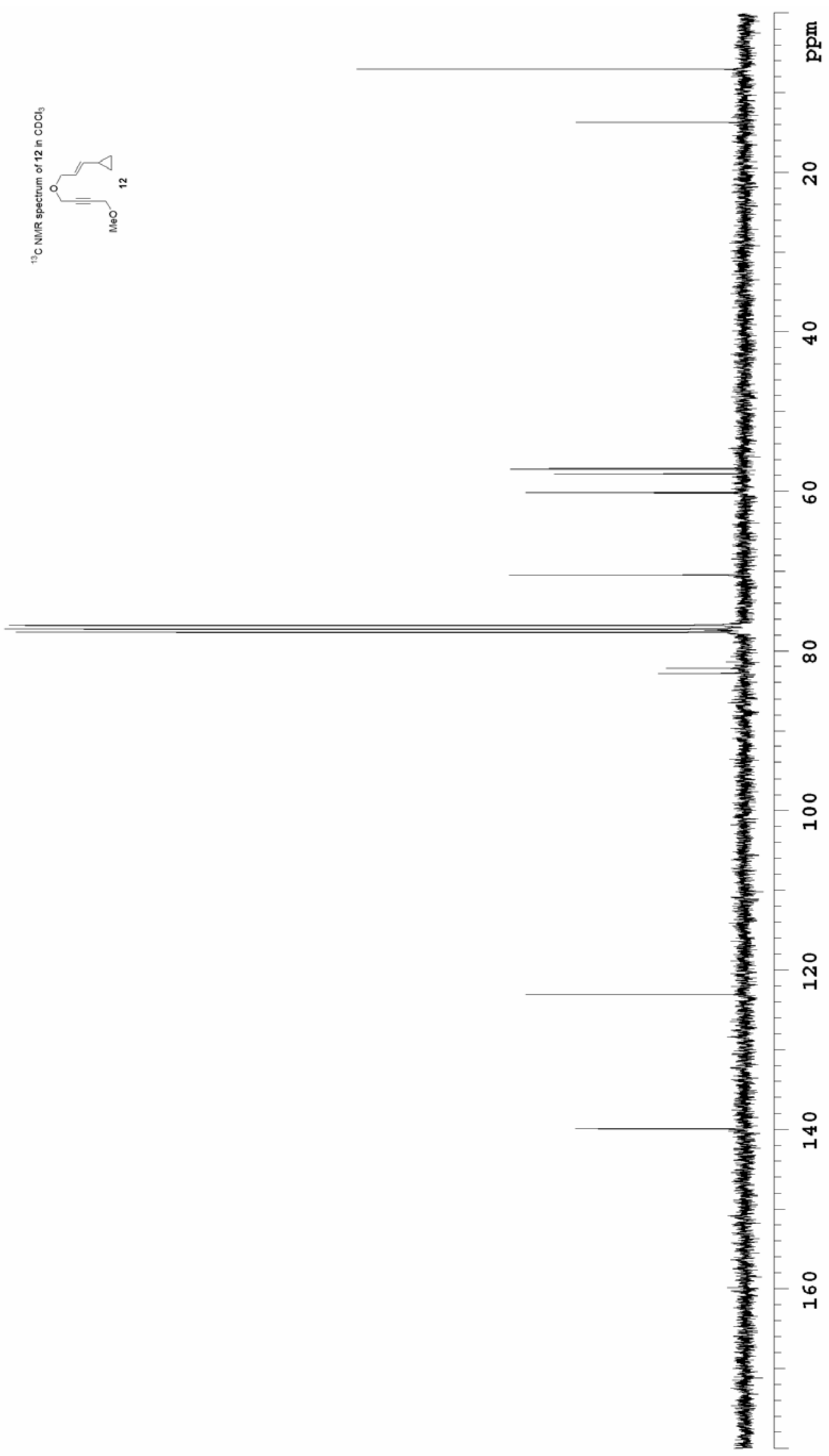


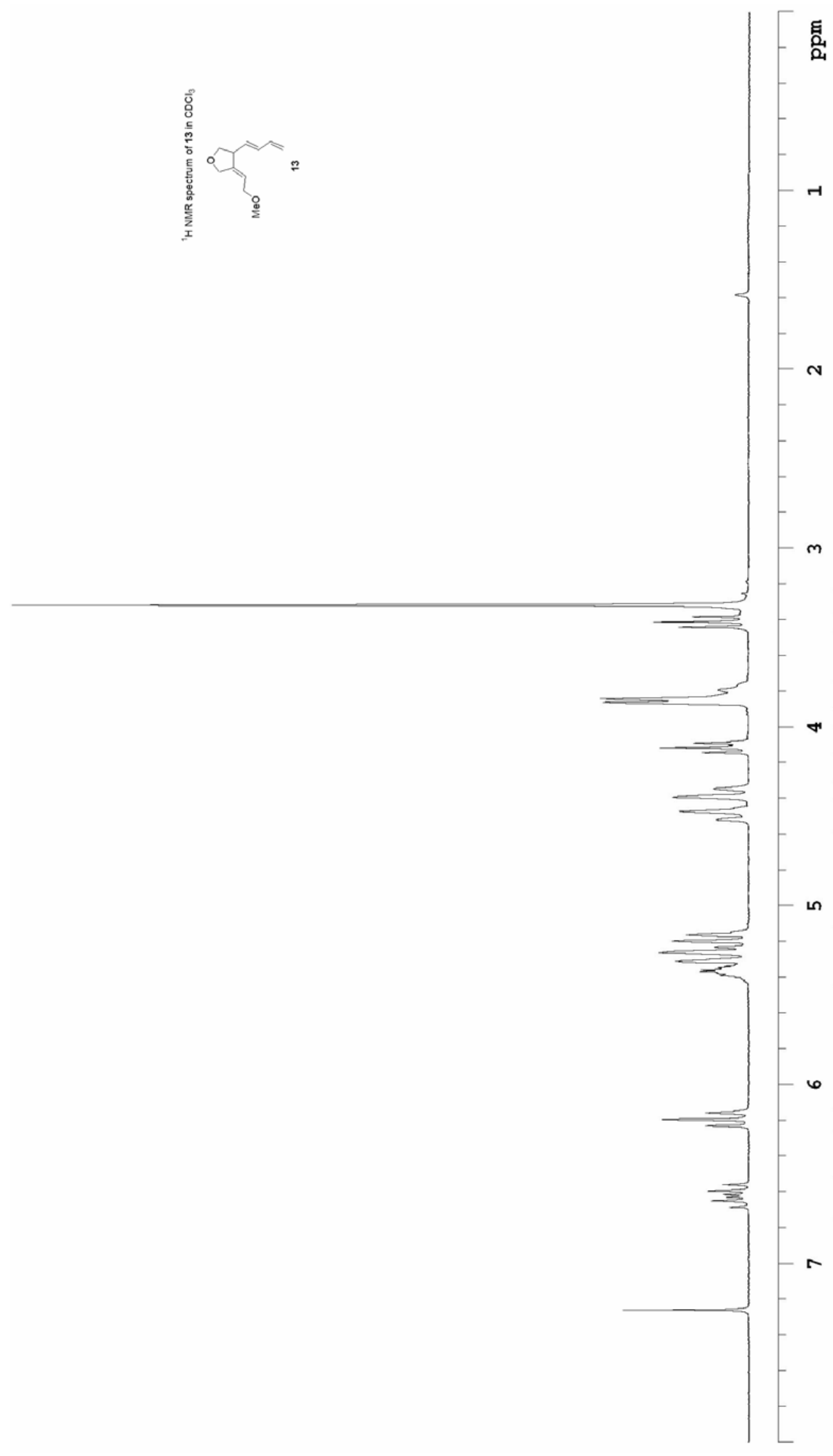




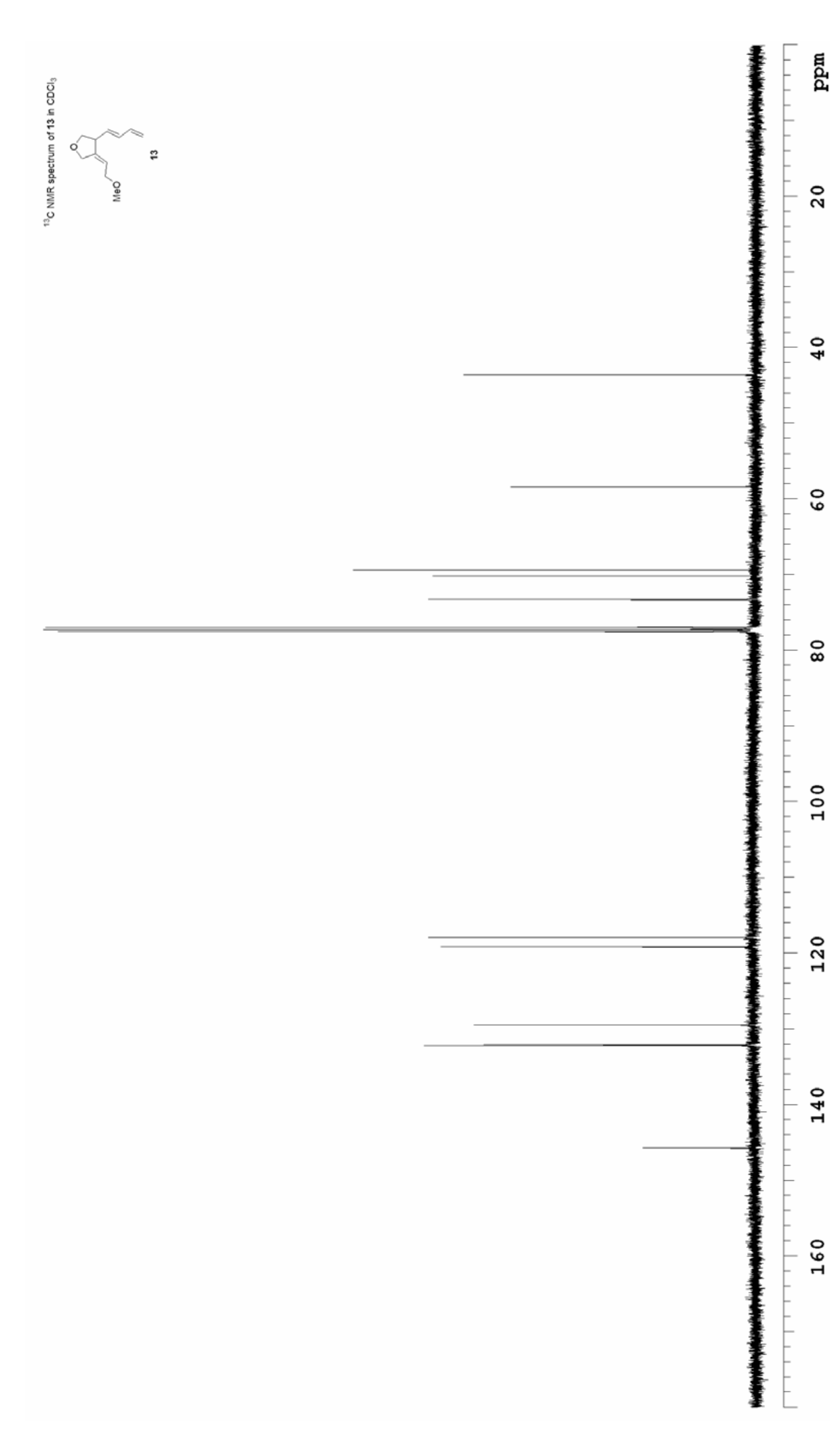



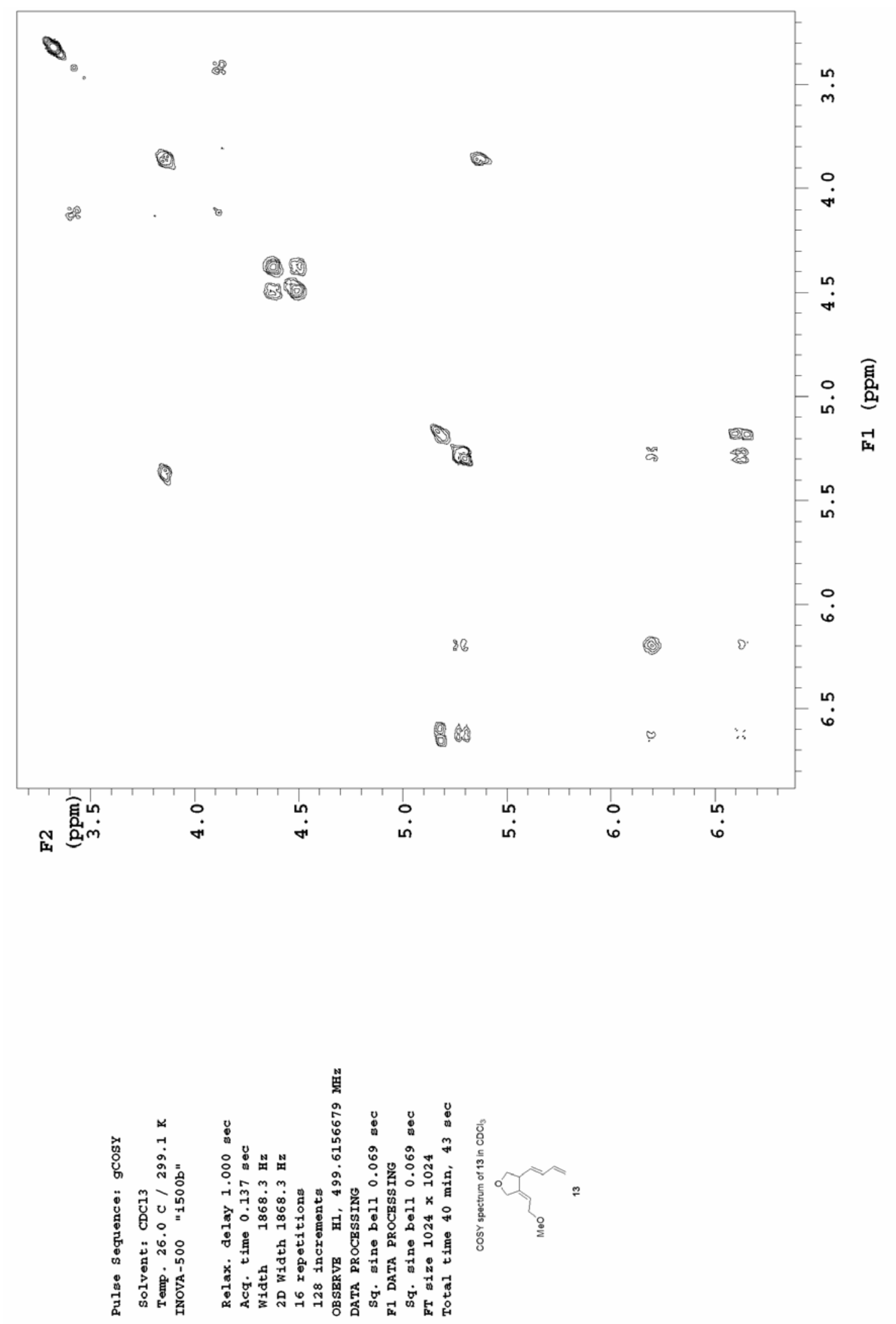

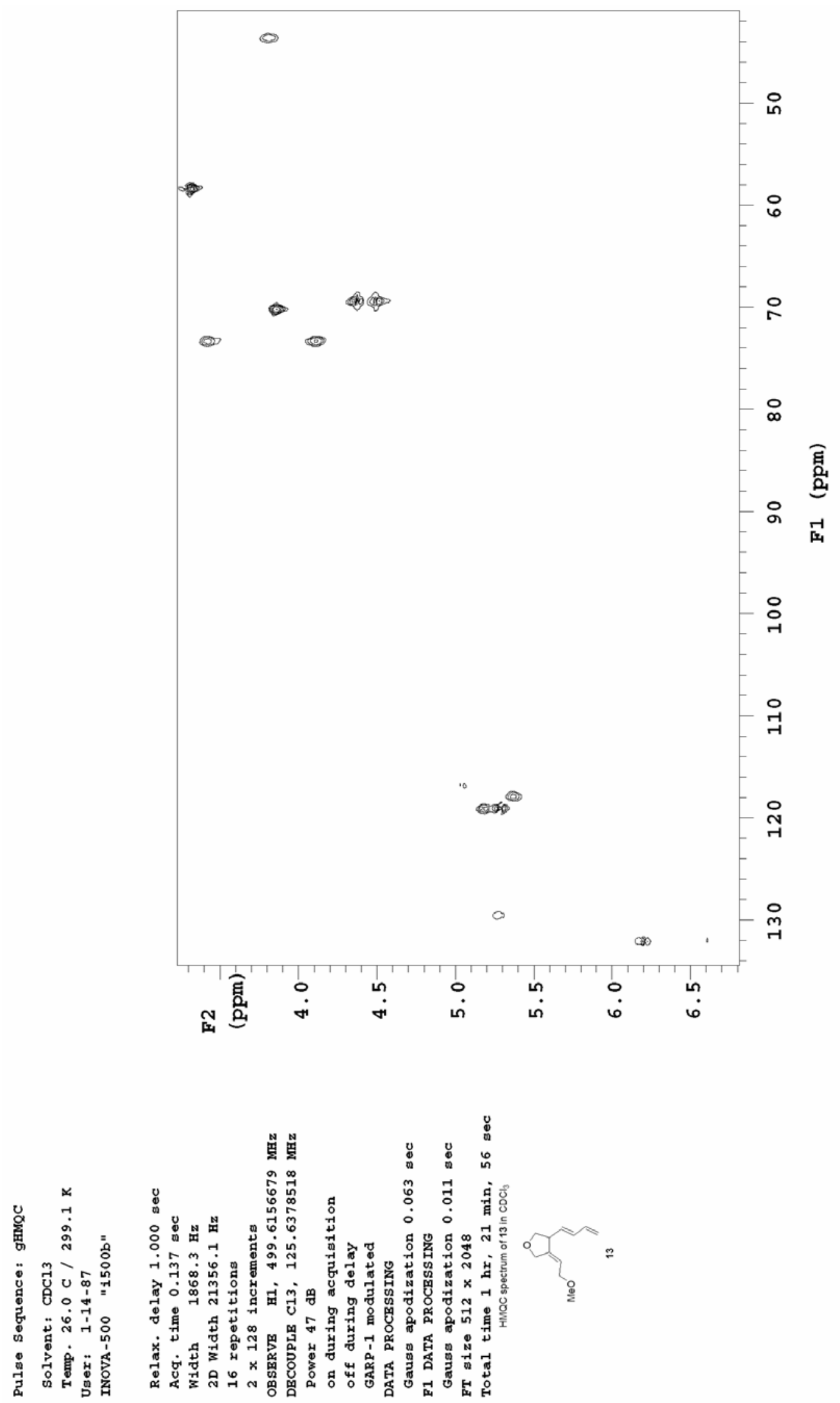

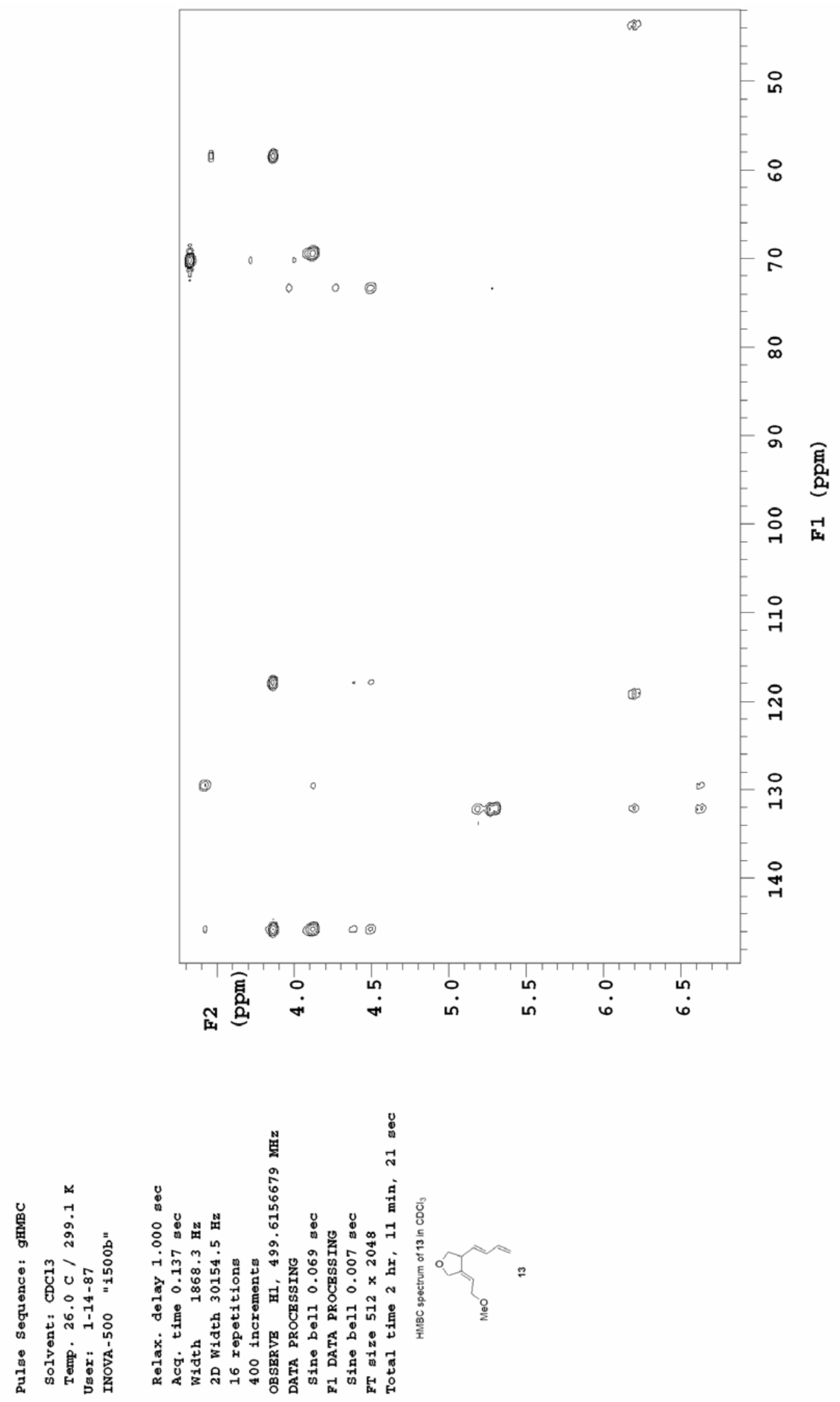


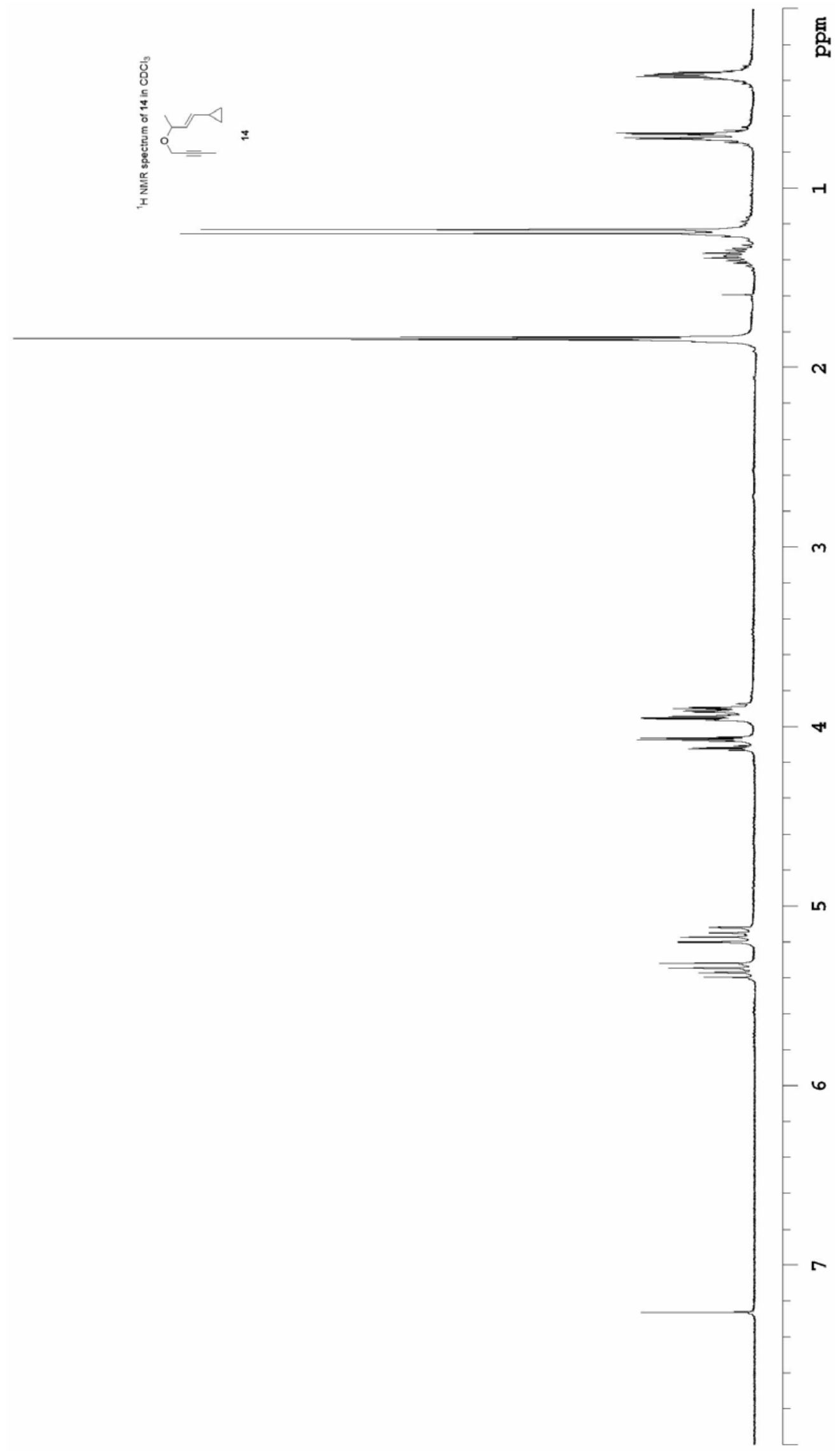



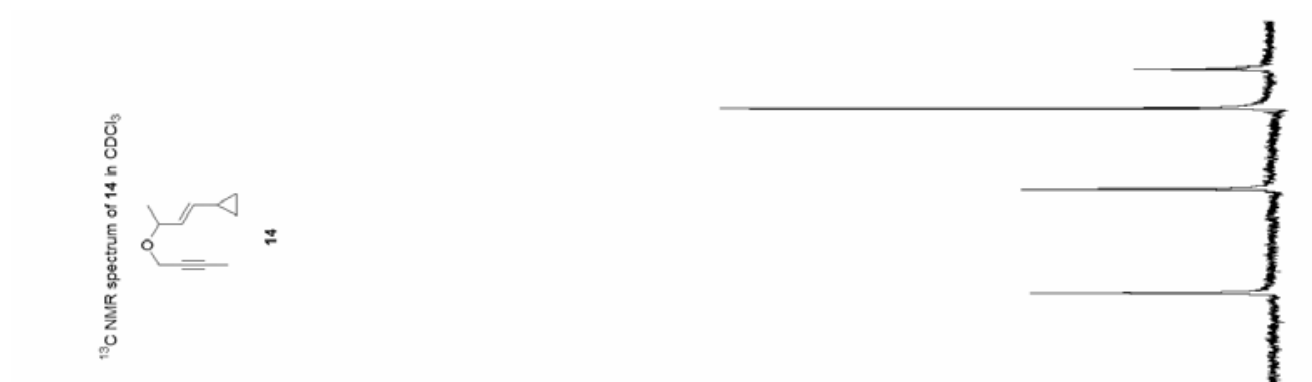

명
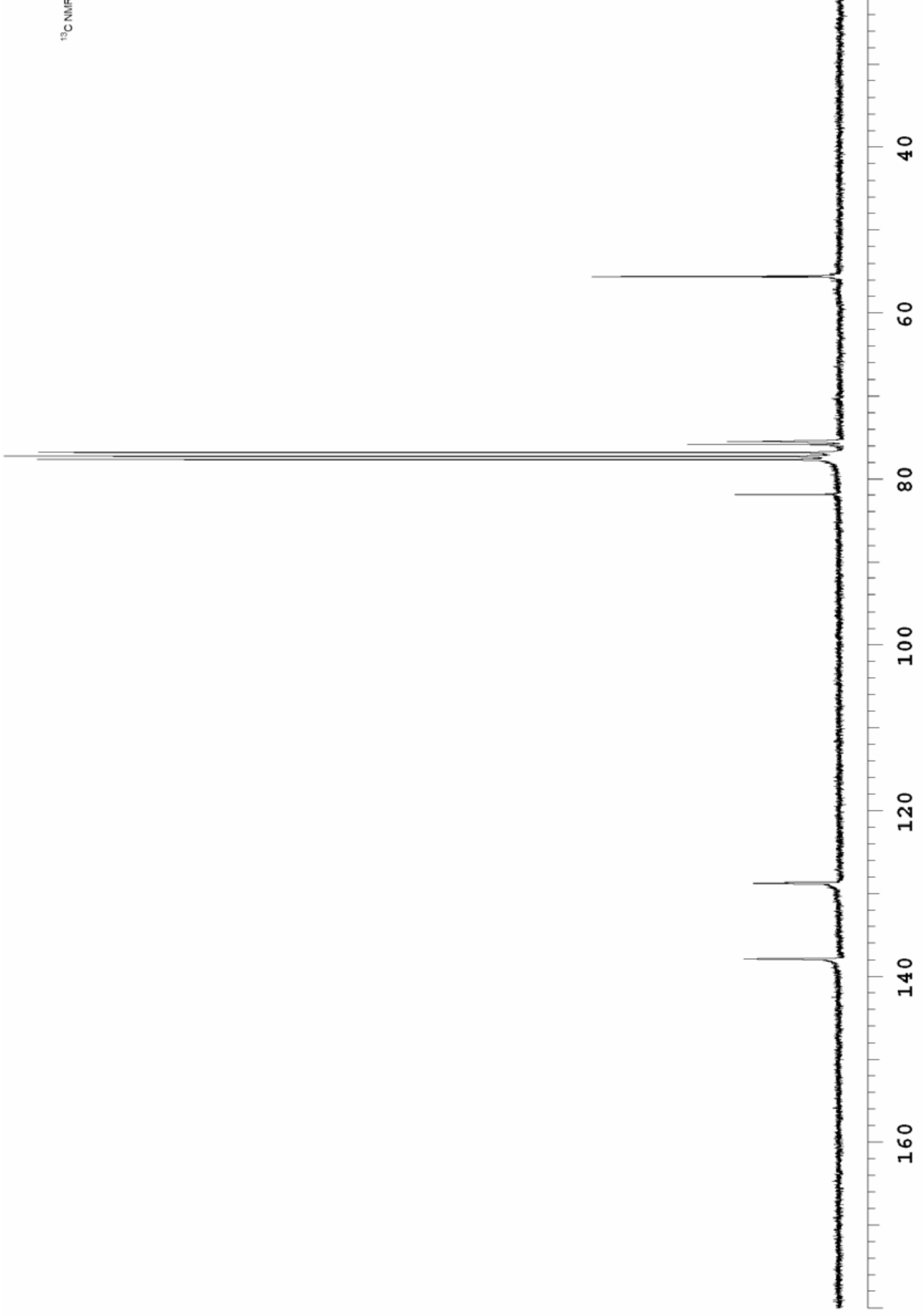


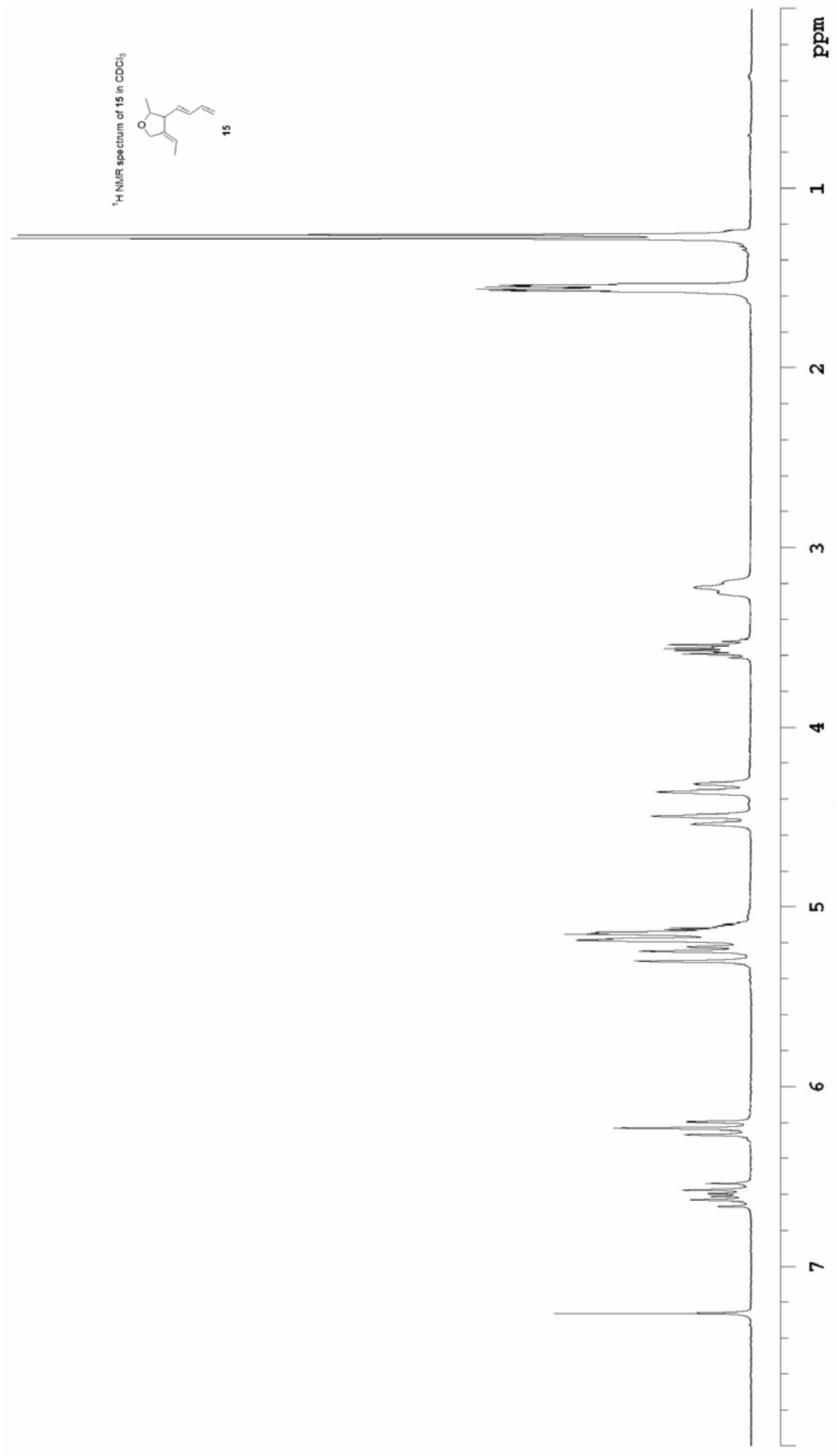



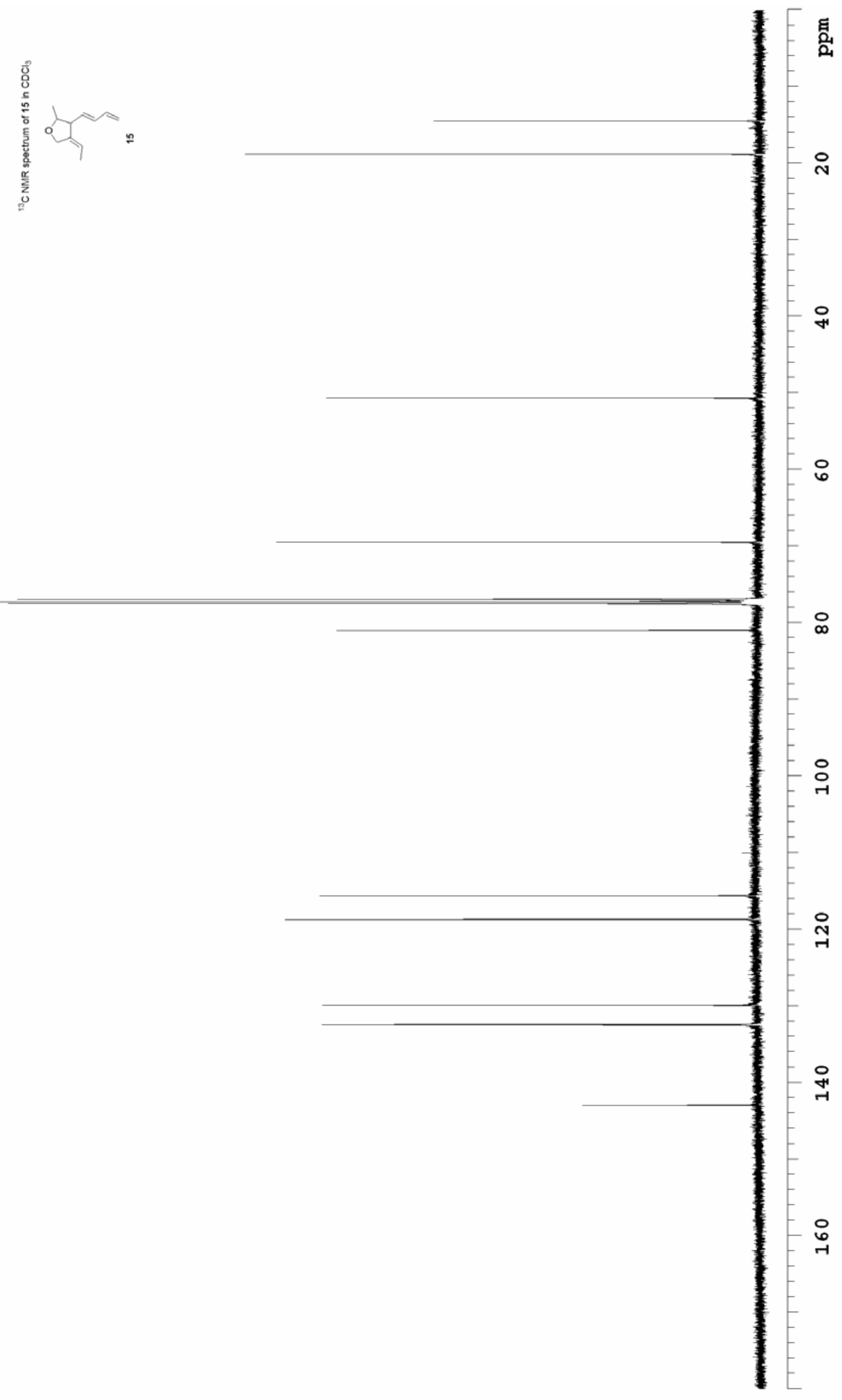

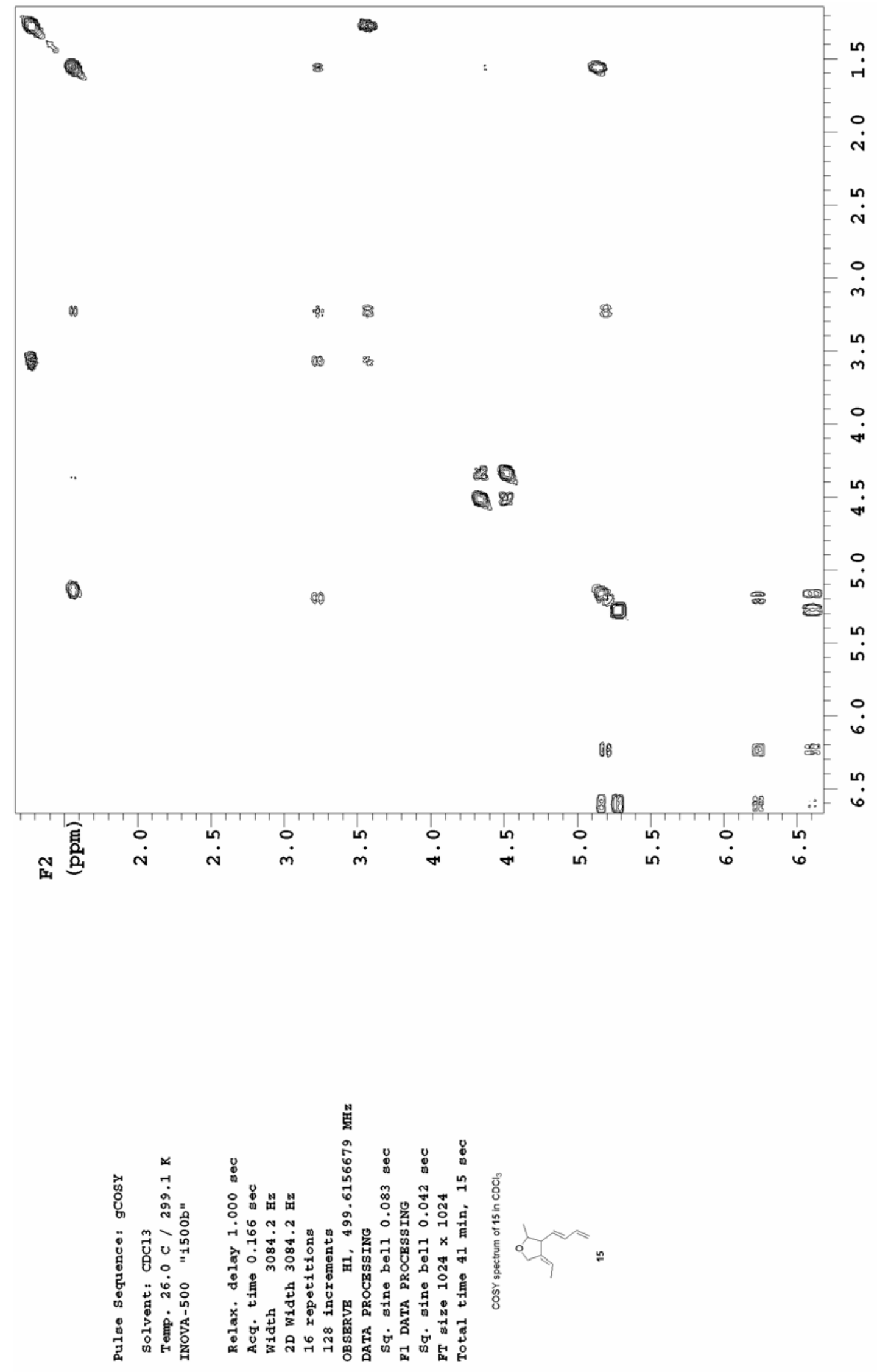

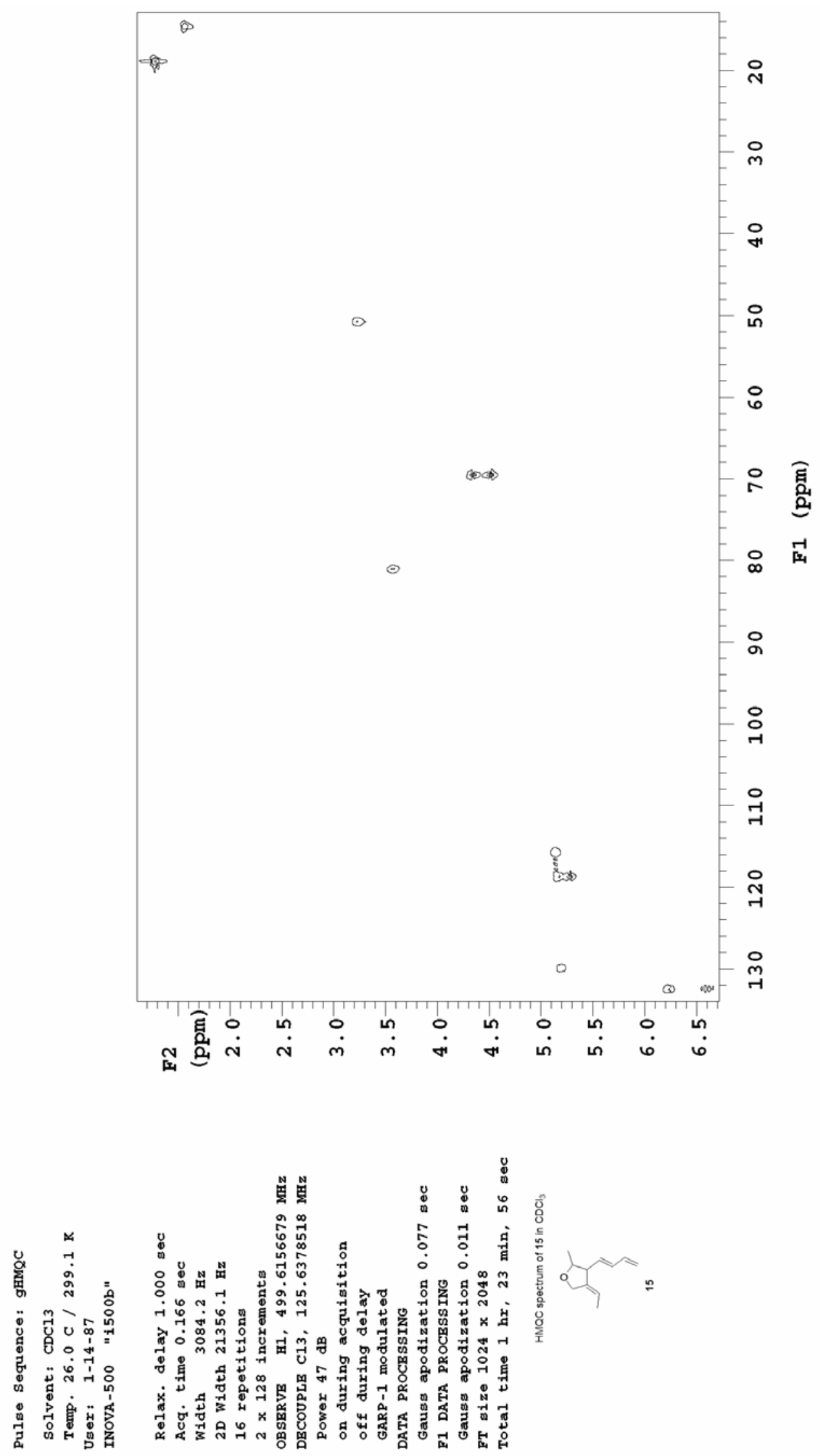

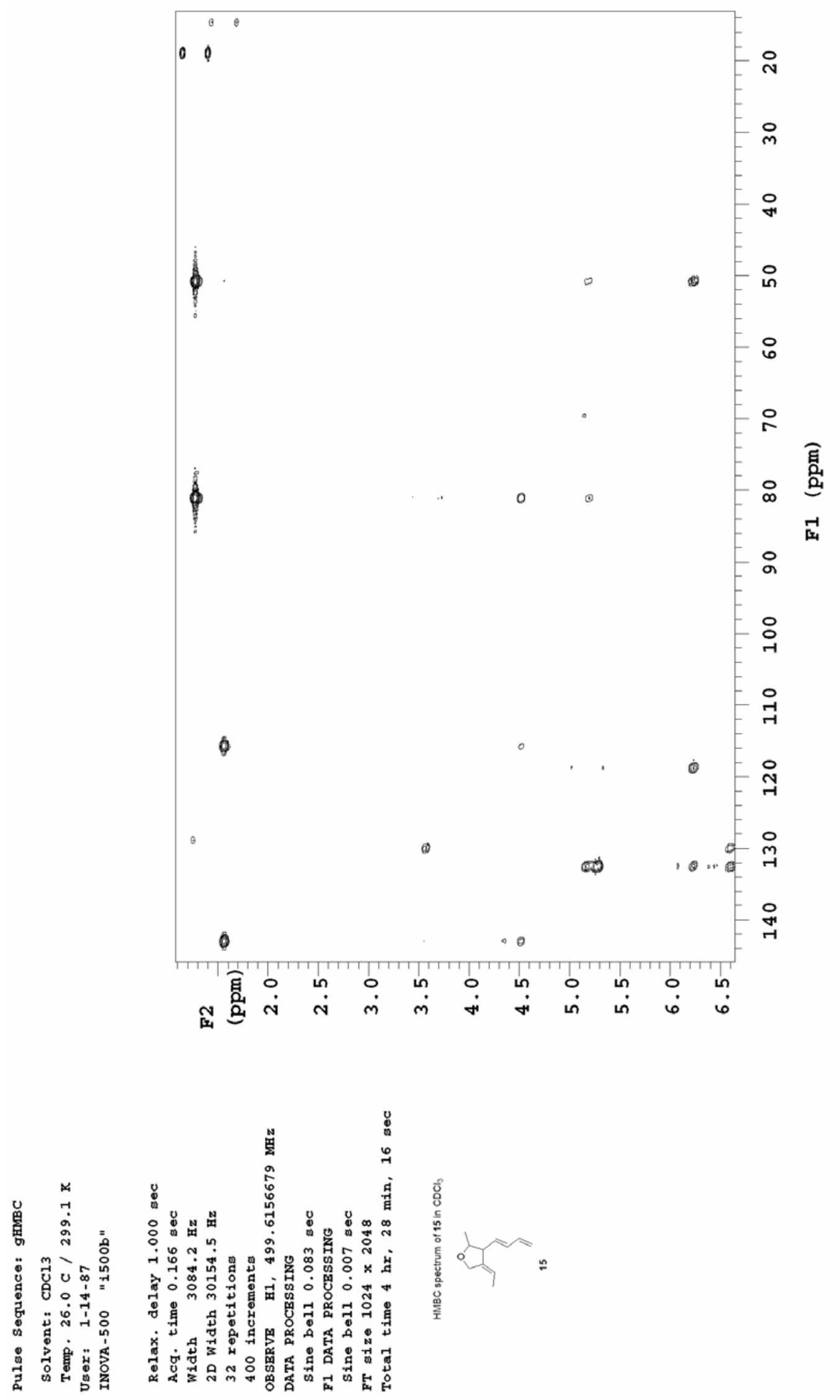

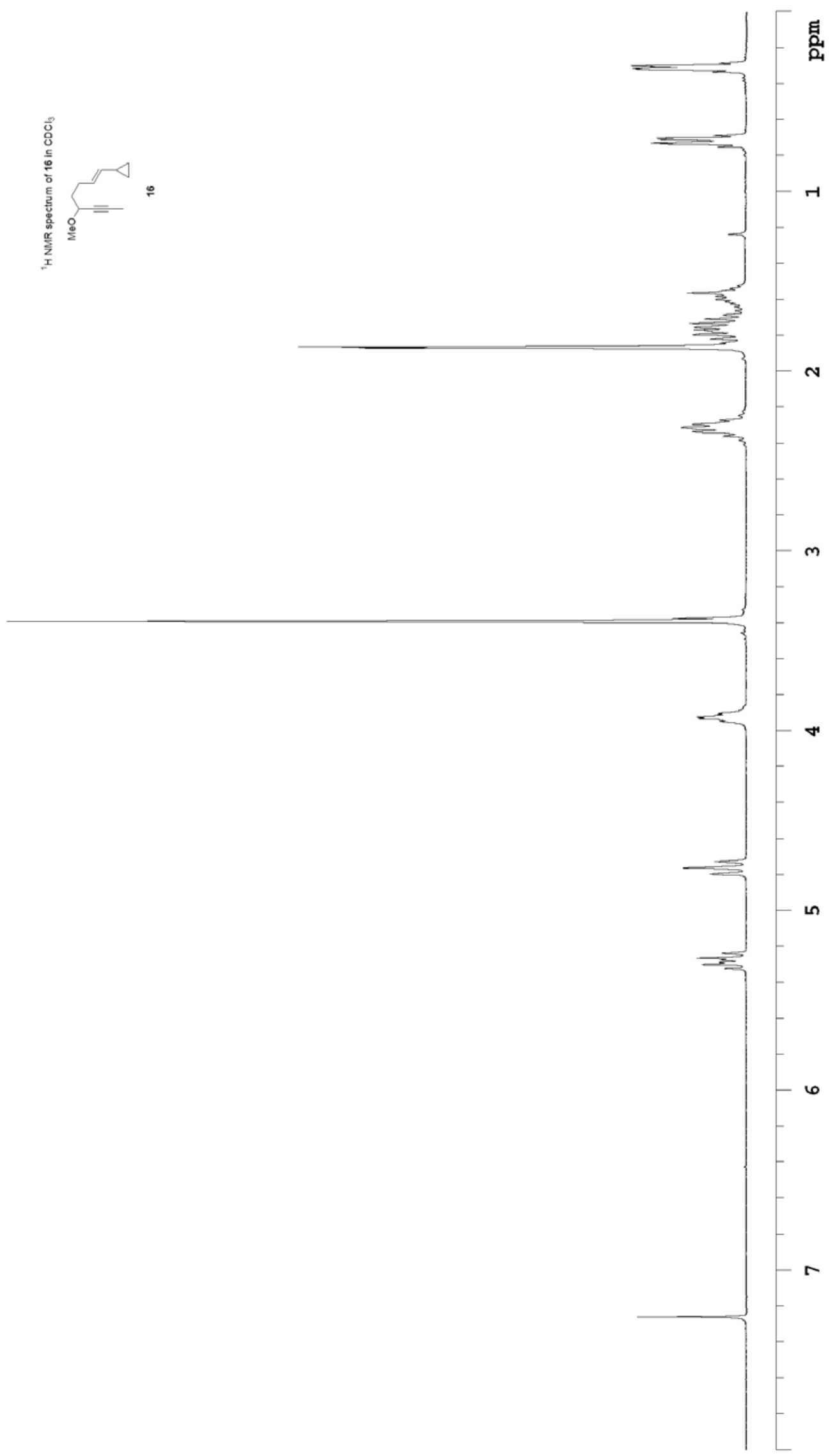

S76 


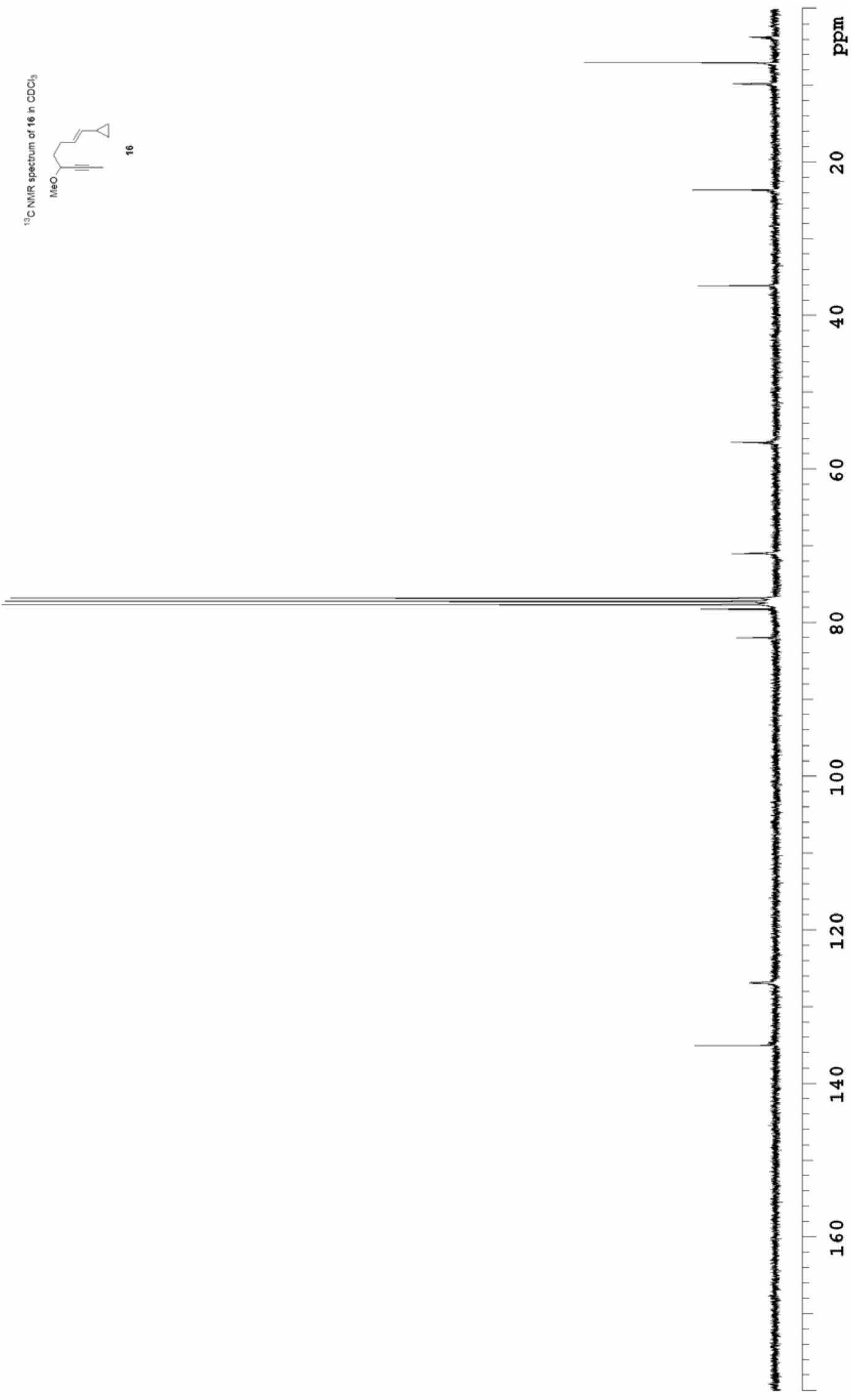




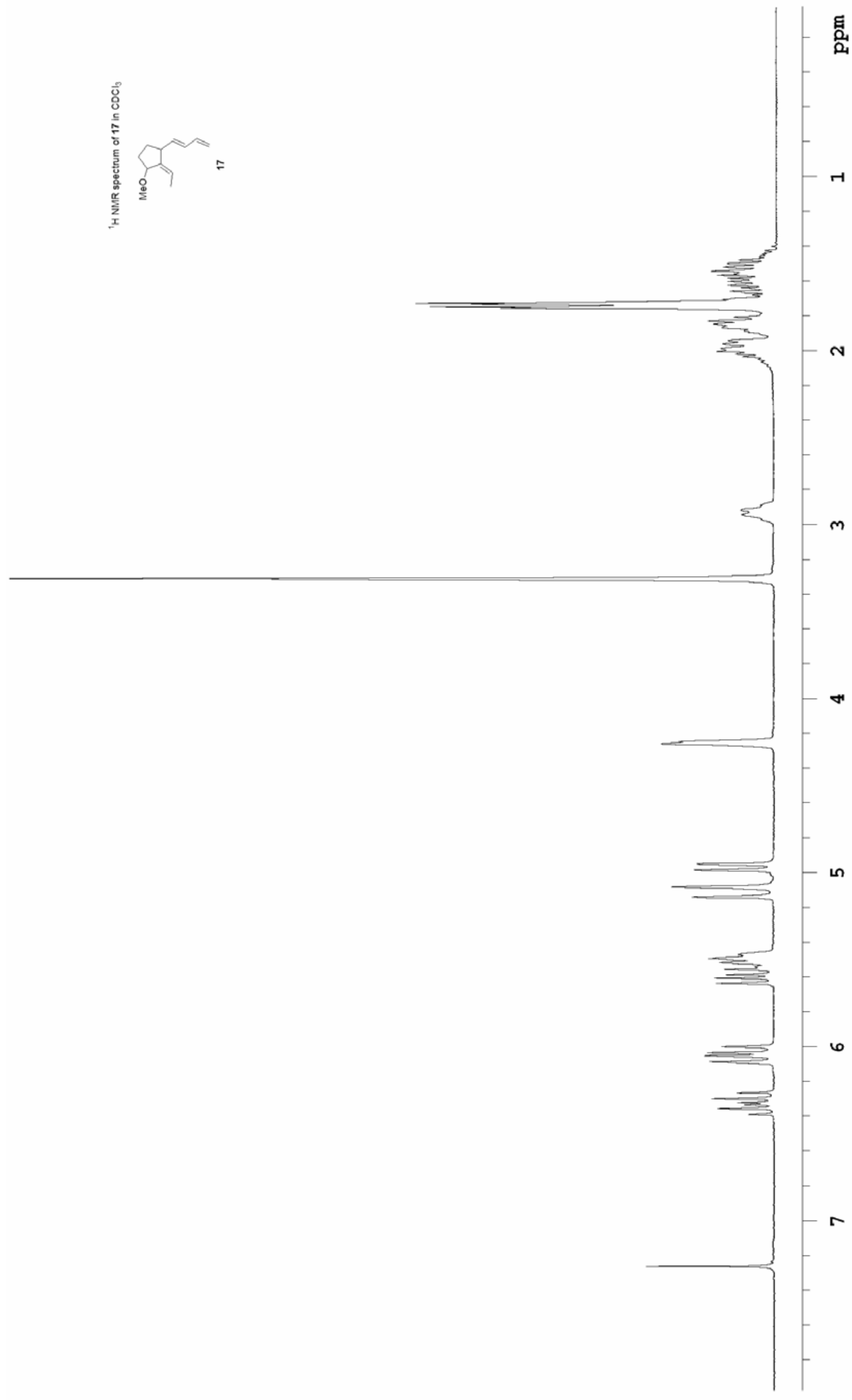




$$
4
$$




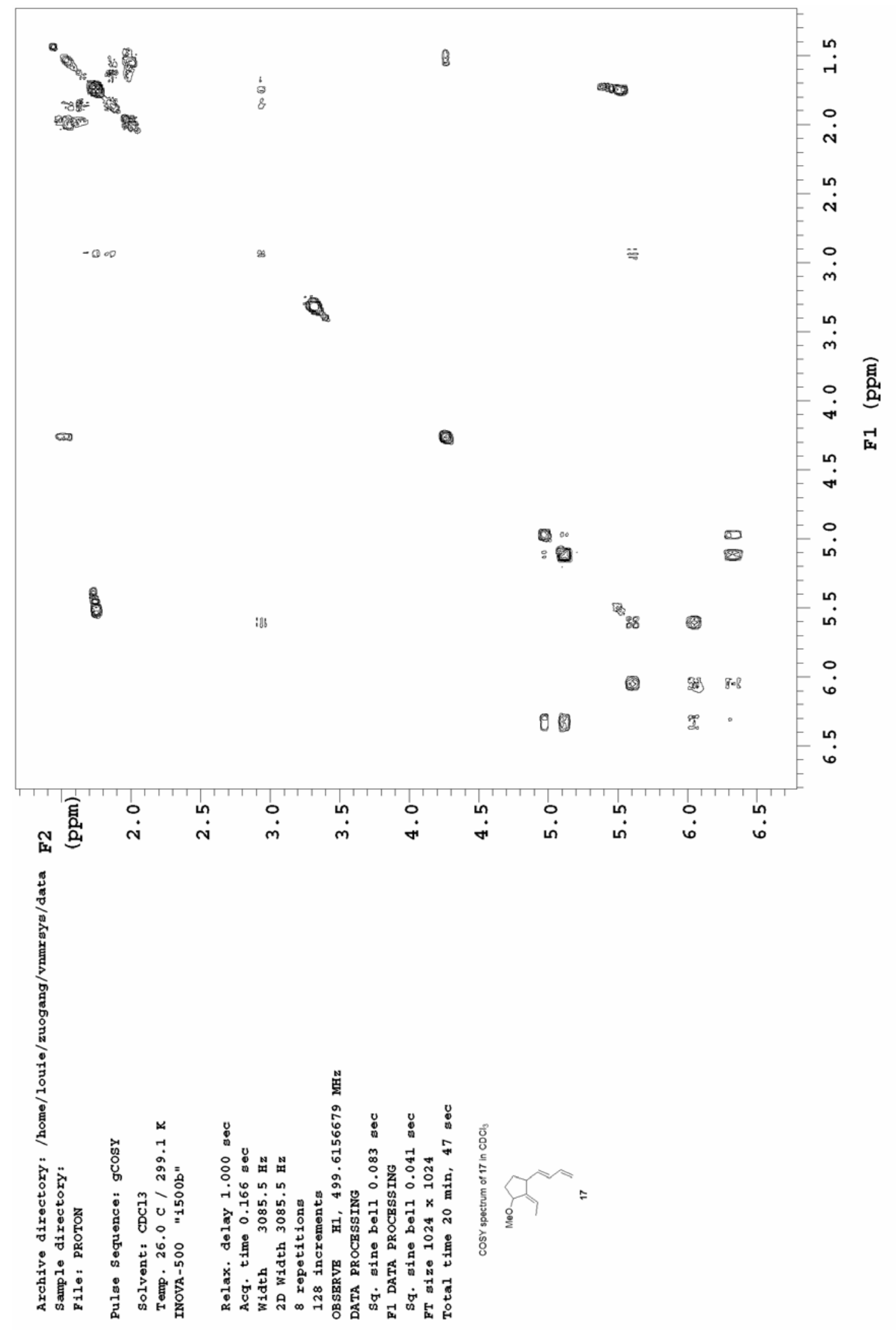



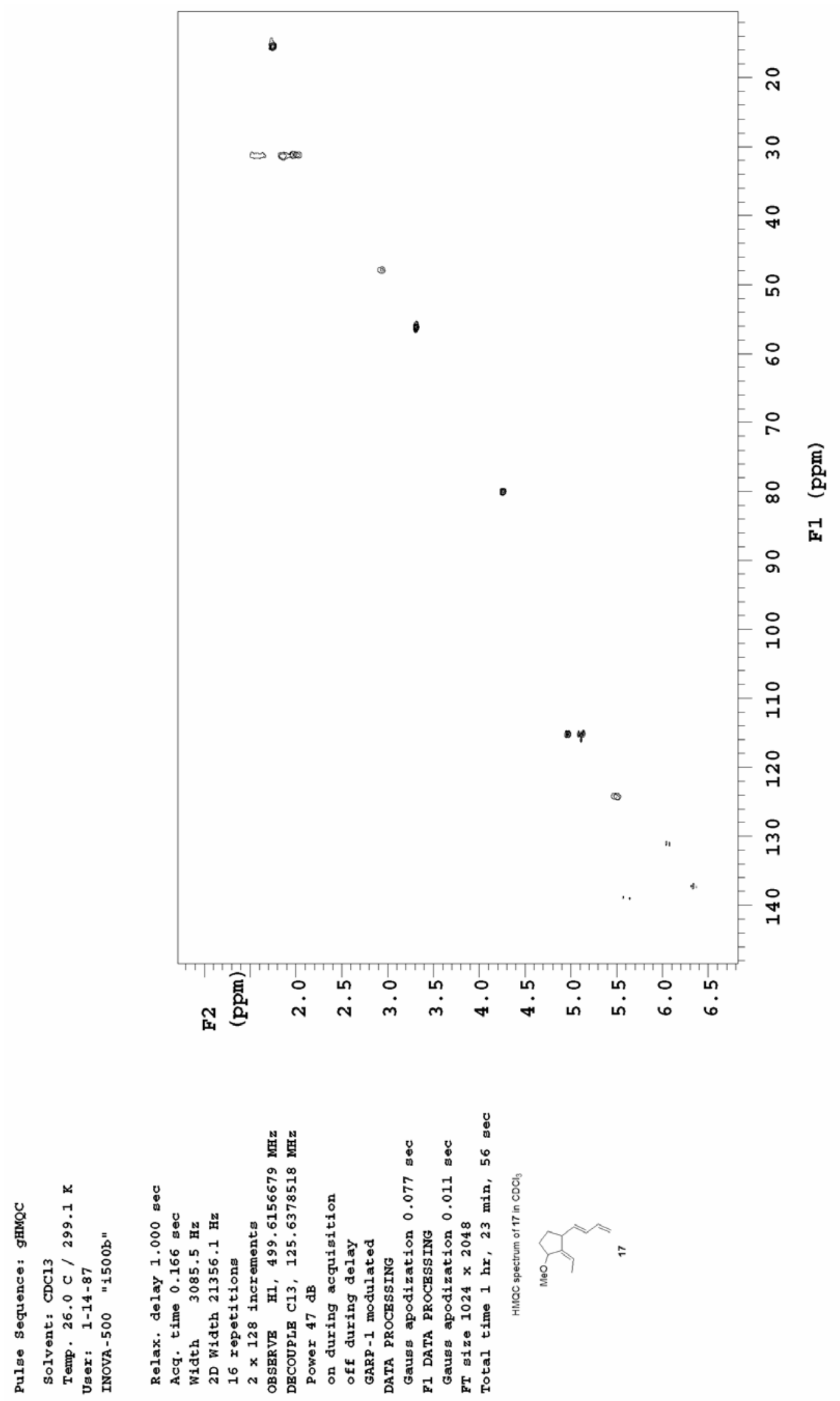

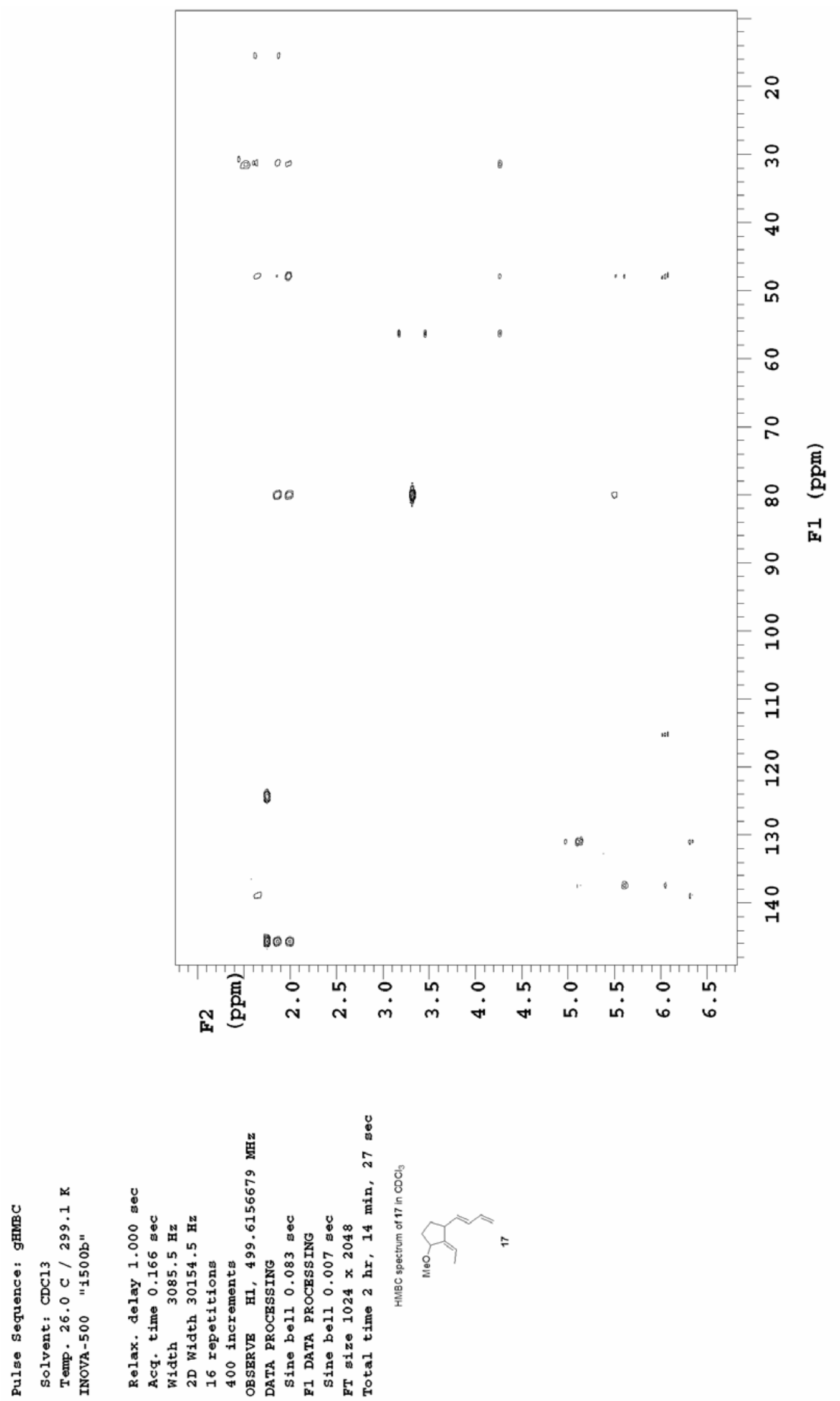

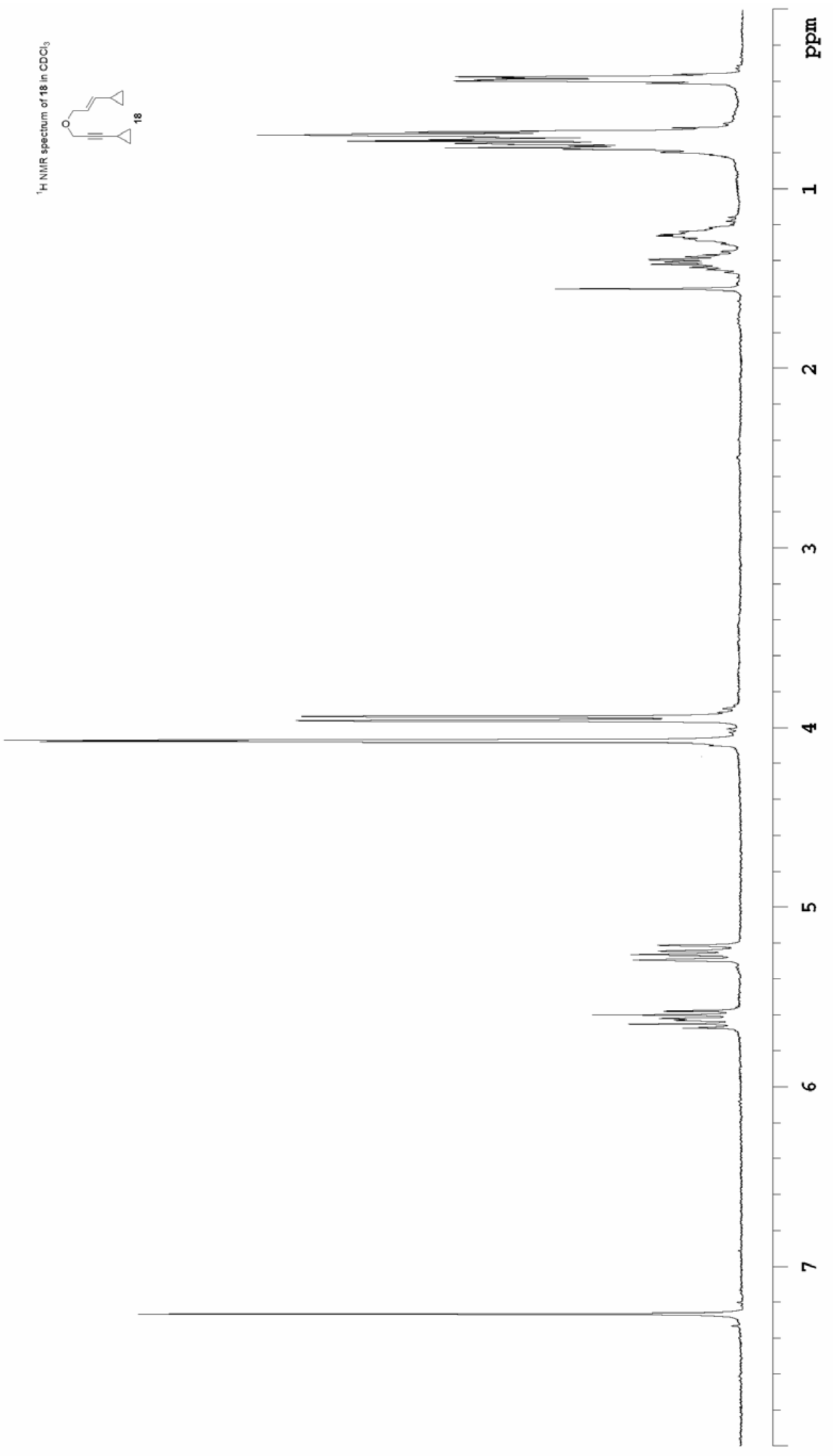


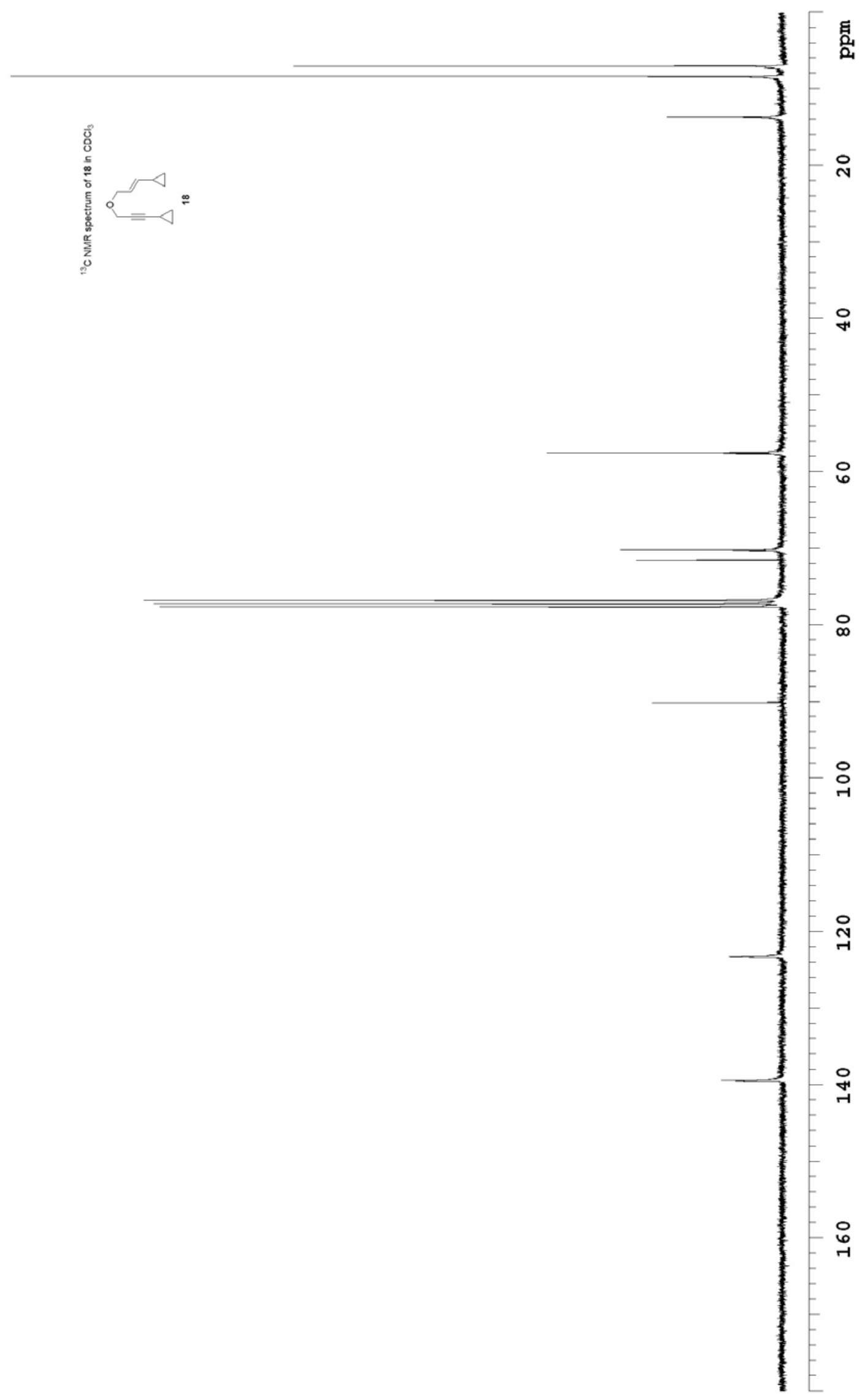




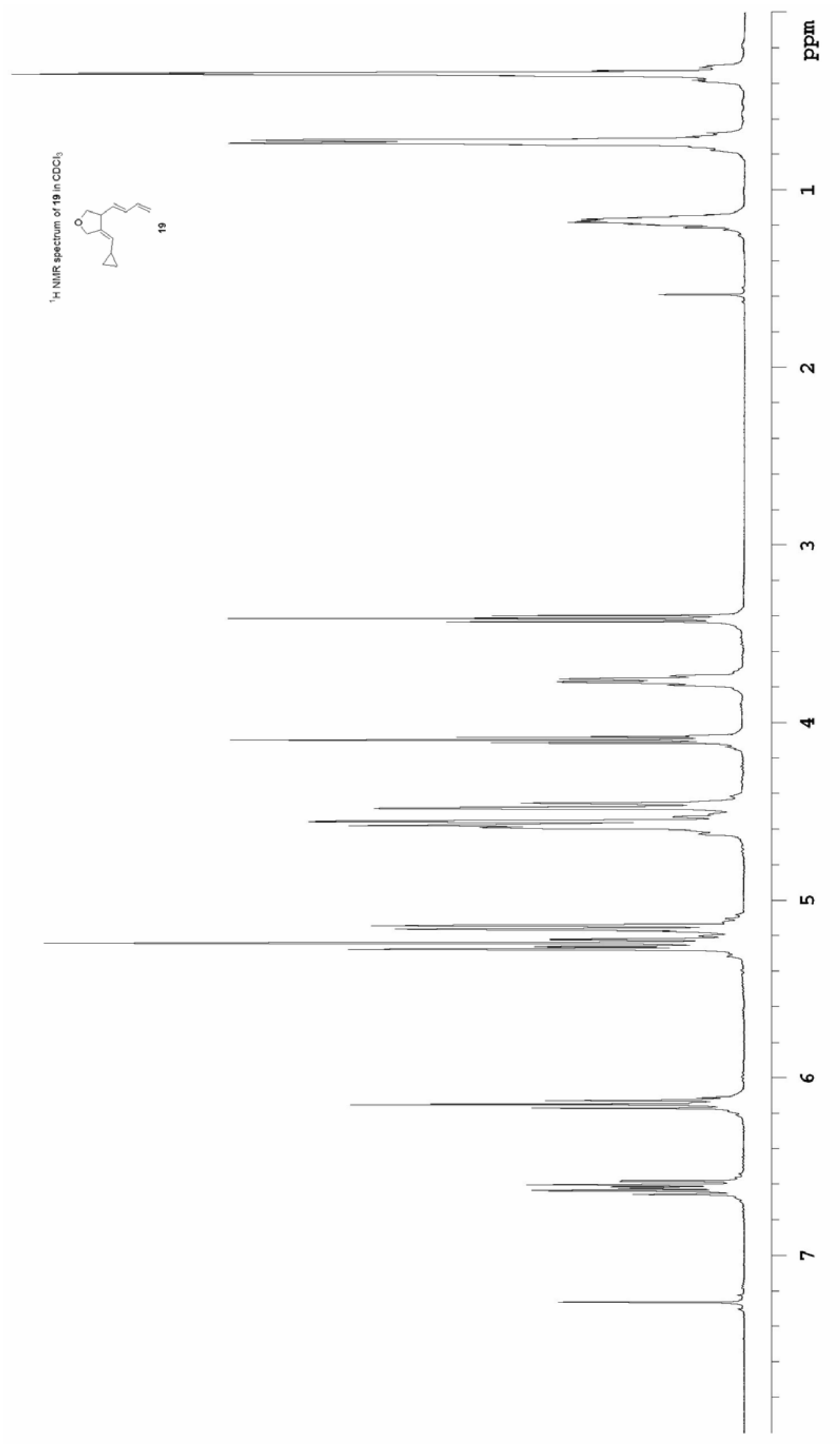




$$
7
$$



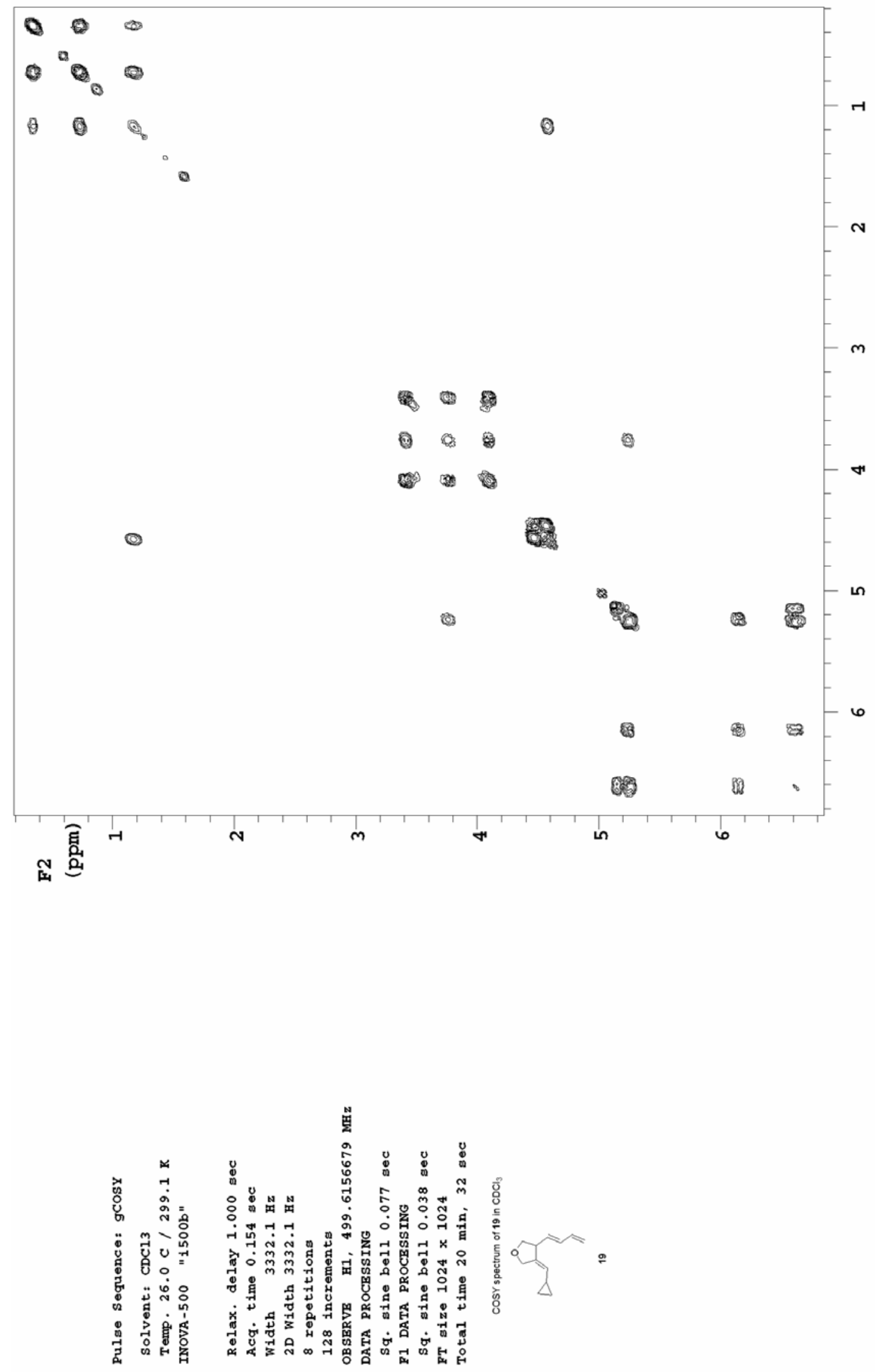

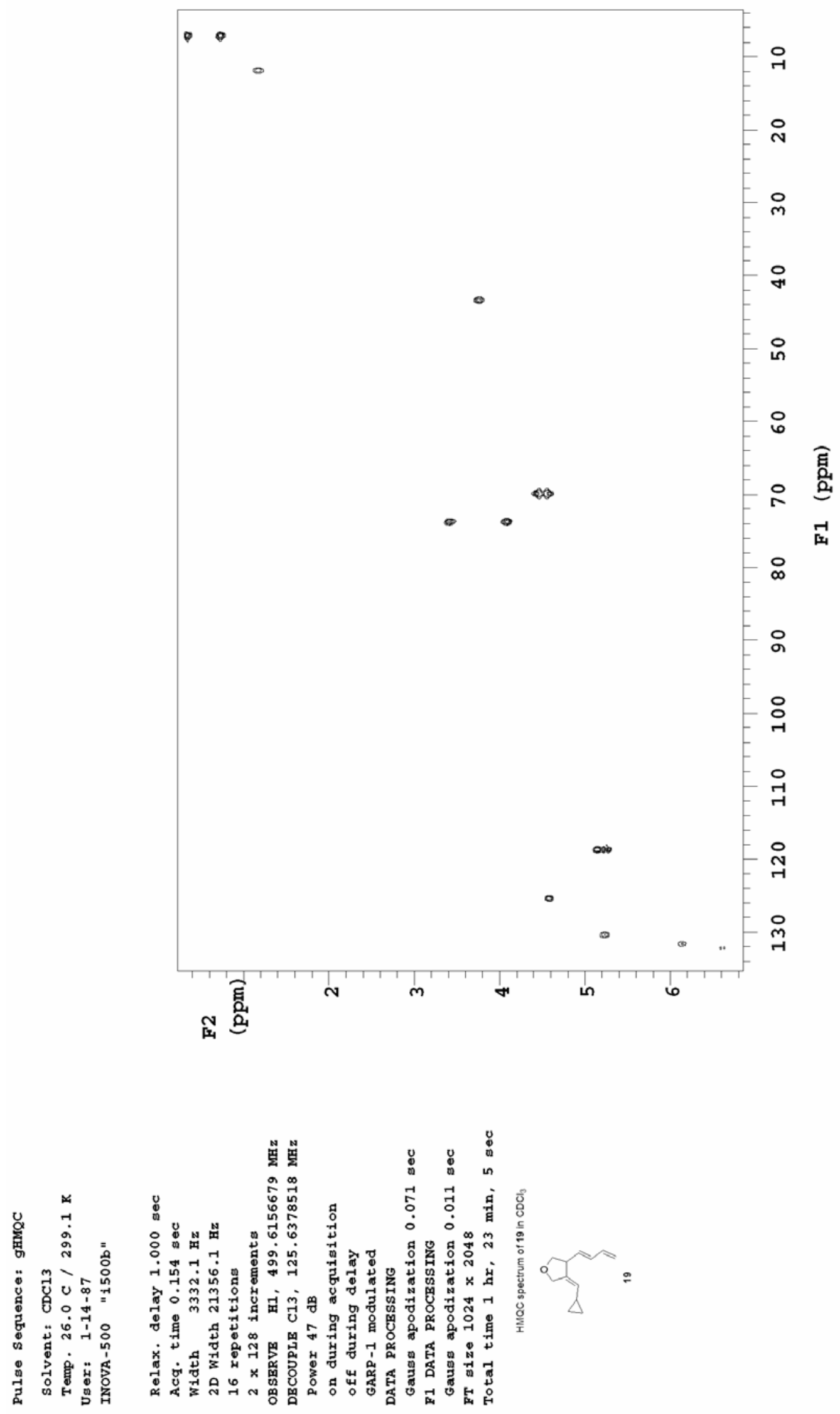

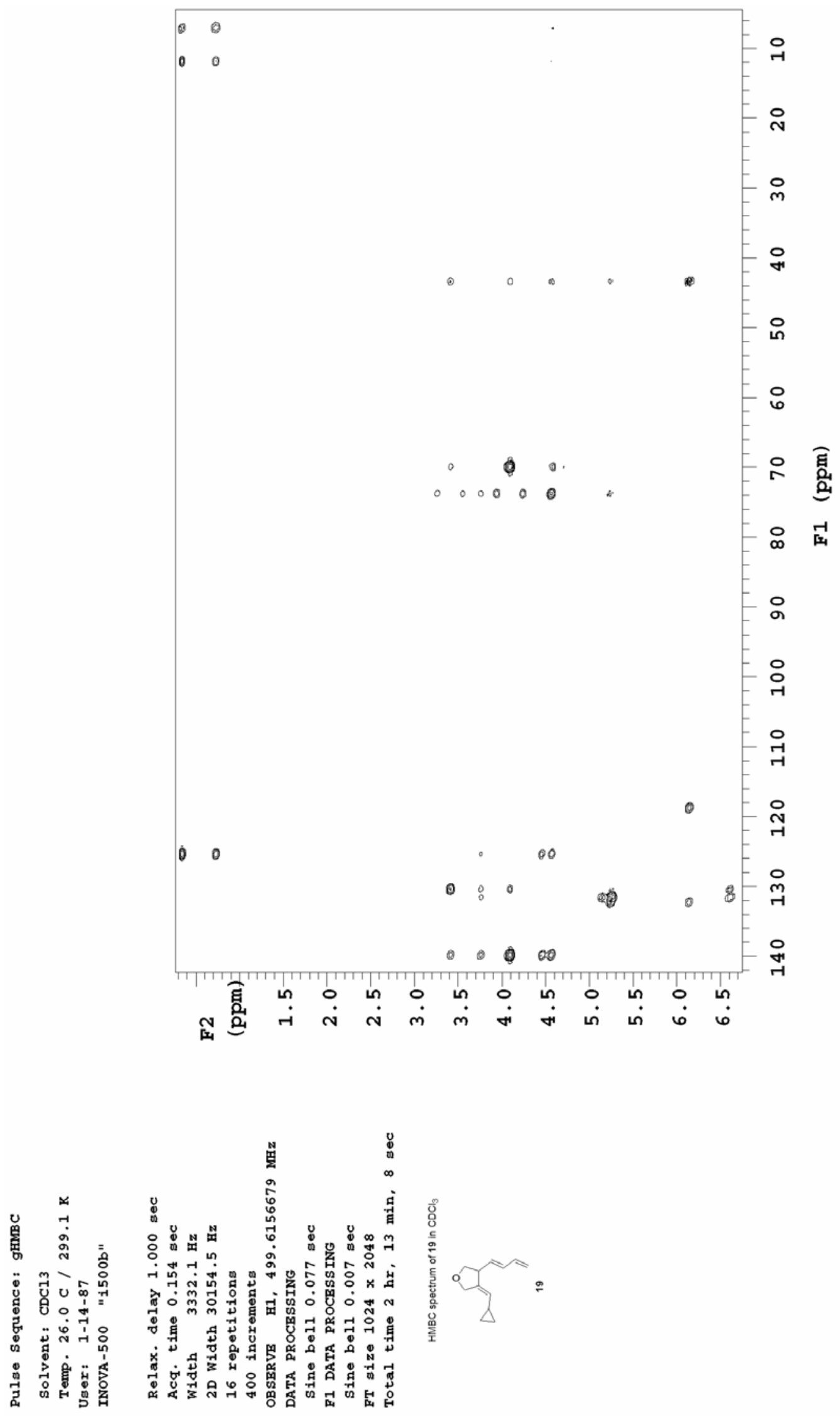

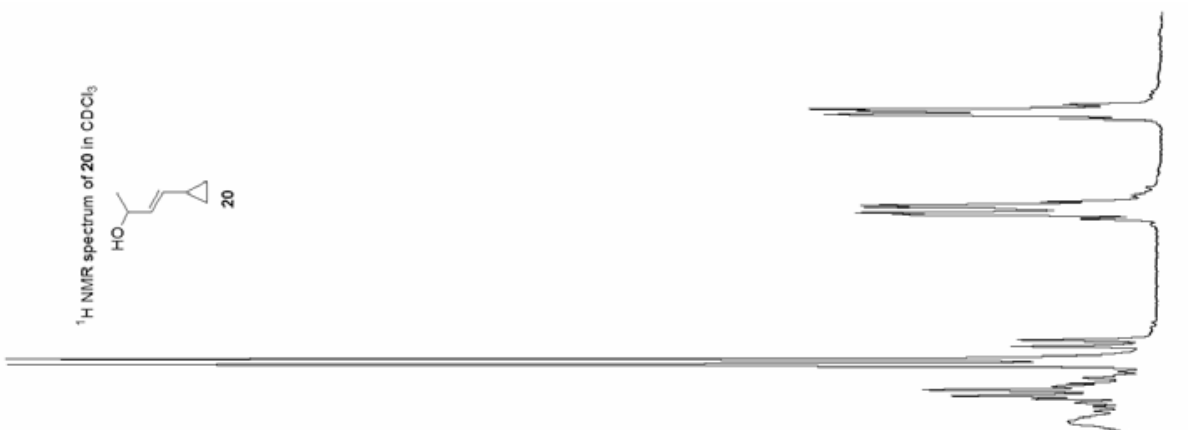

吕

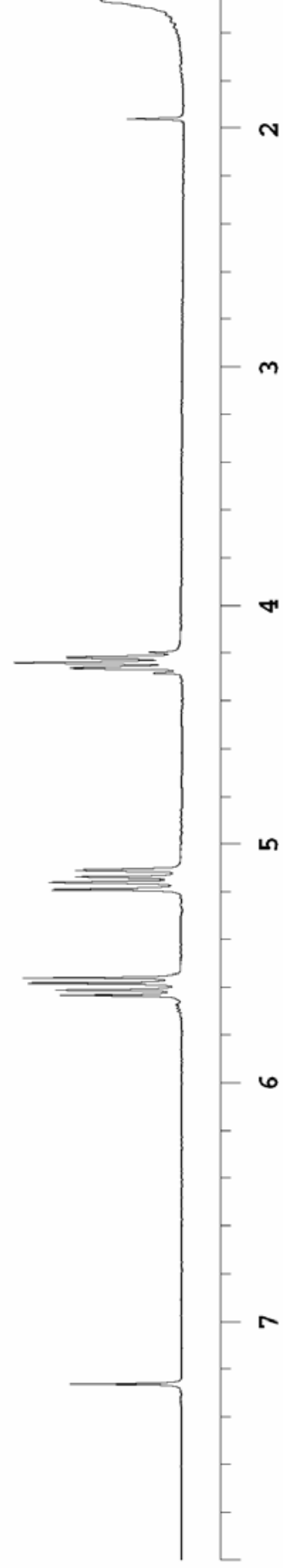




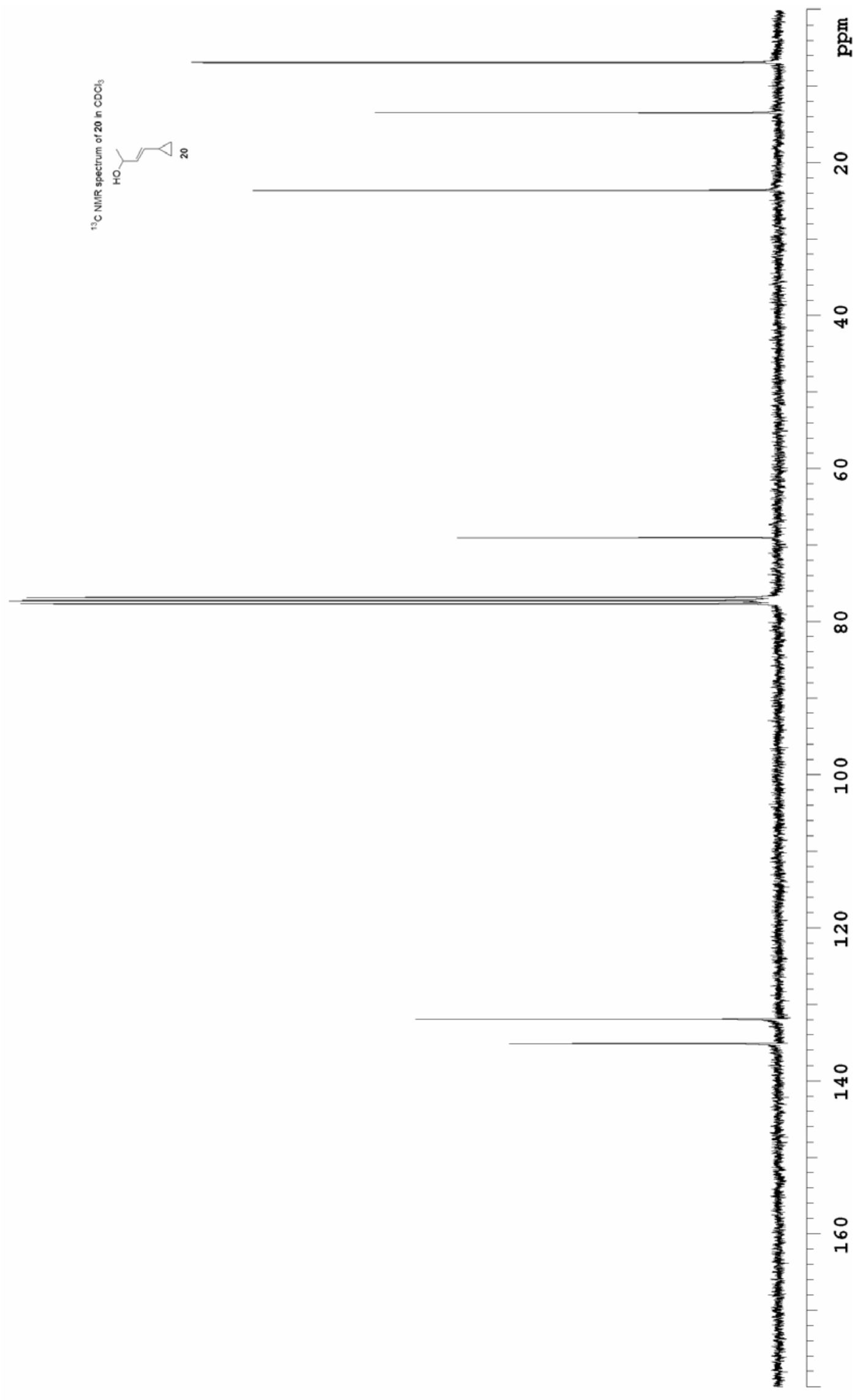




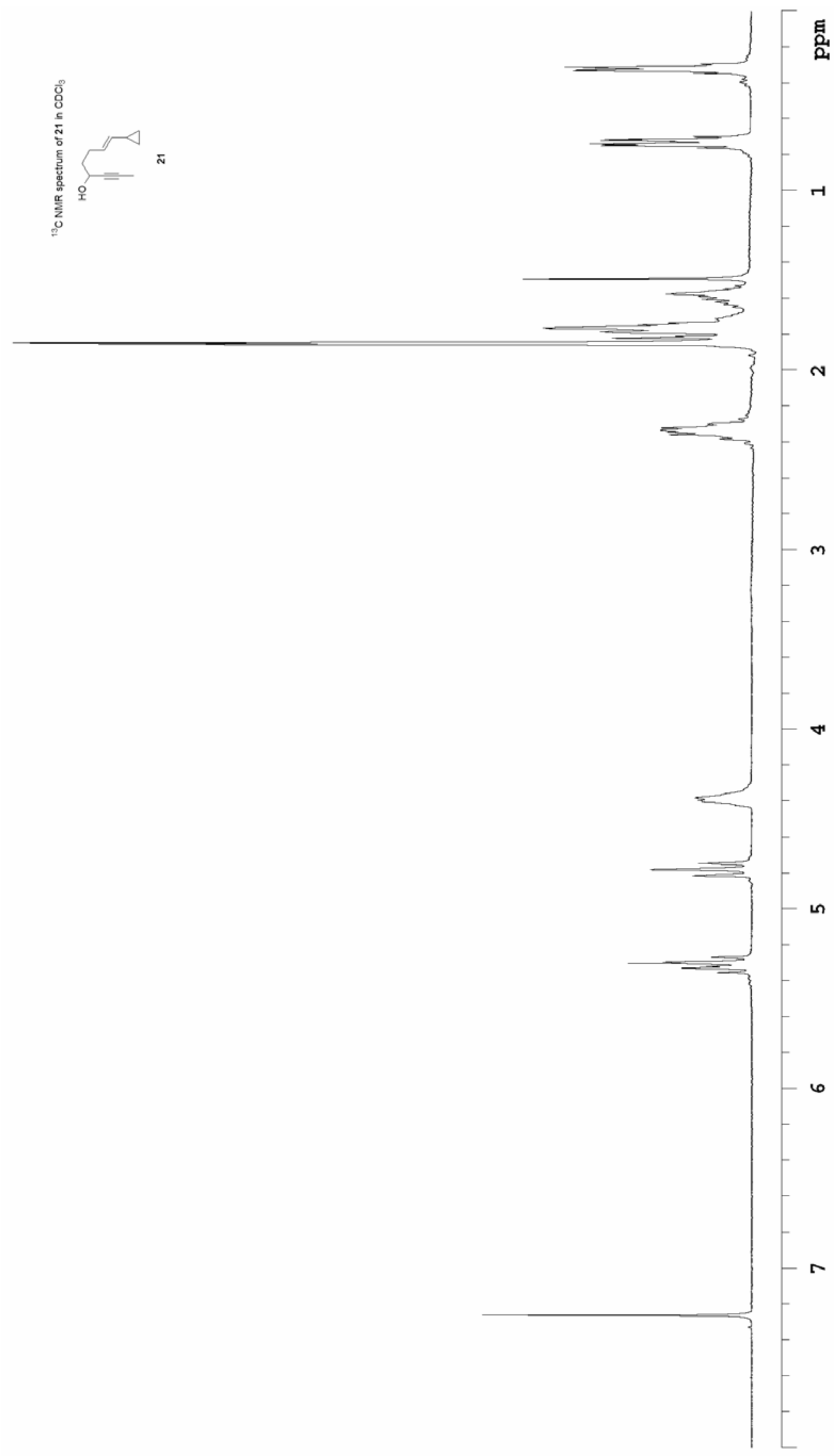



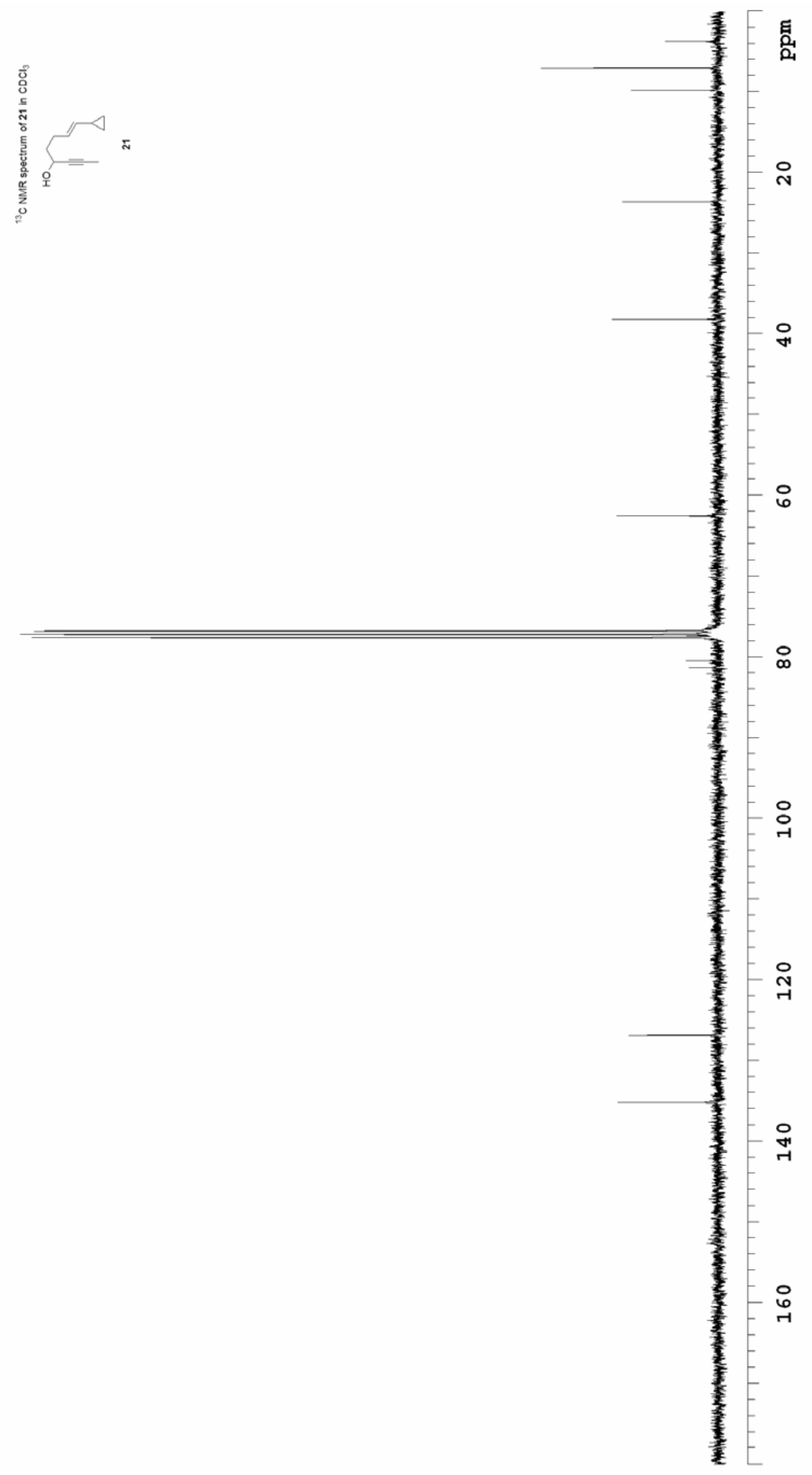